Aus der Klinik für Palliativmedizin

(Prof. Dr. med. F. Nauck)

der Medizinischen Fakultät der Universität Göttingen

\title{
Die Implementierung eines Kinderpalliativzimmers auf einer Normalstation - Eine qualitative Studie
}

\author{
INAUGURAL-DISSERTATION \\ zur Erlangung des Doktorgrades \\ für Zahnheilkunde \\ der Medizinischen Fakultät der \\ Georg-August-Universität zu Göttingen
}

vorgelegt von

Janna Bardt geb. Flach

aus

Darmstadt 
Dekan:

Referent/in:

Ko-Referent/in:

Drittreferent/in:

Datum der mündlichen Prüfung:
Prof. Dr. H.K. Kroemer

Prof. Dr. F. Nauck

Prof. Dr. K. Brockmann

Prof. Dr. R. Mausberg

24.07.2017 
Hiermit erkläre ich, die Dissertation mit dem Titel "Die Implementierung eines Kinderpalliativzimmers auf einer Normalstation - Eine qualitative Studie" eigenständig angefertigt und keine anderen als die von mir angegebenen Quellen und Hilfsmittel verwendet zu haben.

Göttingen, den 


\section{Inhaltsverzeichnis}

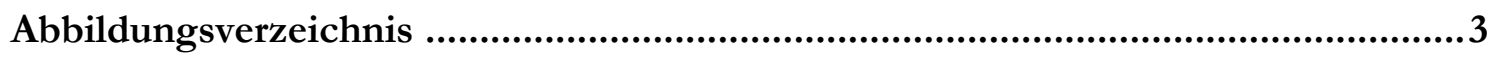

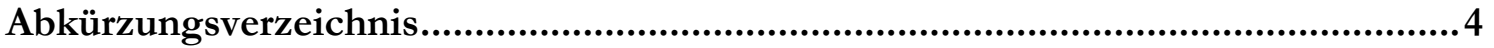

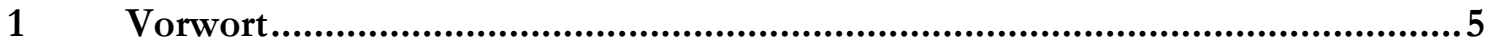

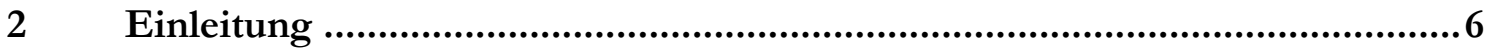

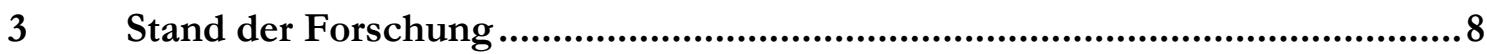

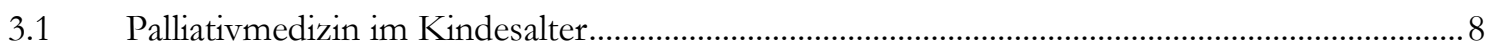

3.1.1 Sterblichkeit von Kindern - Häufigkeit in verschiedenen Altersgruppen ................................. 8

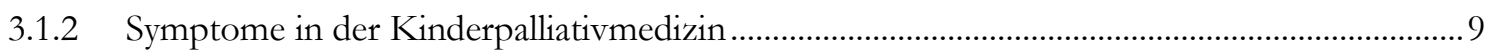

3.2 Das Versorgerteam in der Kinderpalliativmedizin................................................................

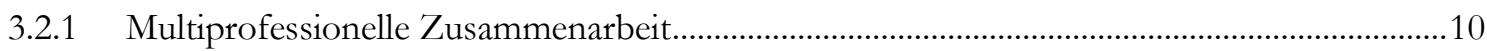

3.2.2 Emotionale Belastung der Versorgerinnen und Versorger........................................................11

3.3 Unterschiede in der Palliativversorgung von Erwachsenen und Kindern ...............................12

3.4 Die Situation der Familie des erkrankten Kindes..................................................................13

3.4.1 Emotionale Belastung von Eltern und Geschwistern ..............................................................13

3.4.2 Die Bedeutung der Beziehung zwischen der Familie und den Behandlern.............................14

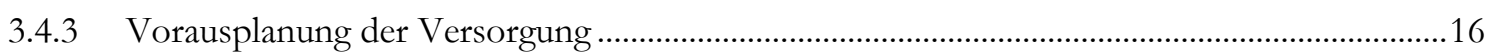

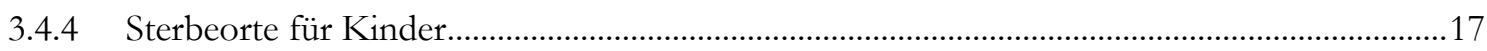

3.5 Versorgungsstrukturen der pädiatrischen Palliativversorgung................................................18

3.5.1 Pädiatrische Palliativversorgungversorgung in Kliniken ...........................................................19

3.6 Das Kinderpalliativzentrum Göttingen mit Kinderpalliativzimmer ........................................19

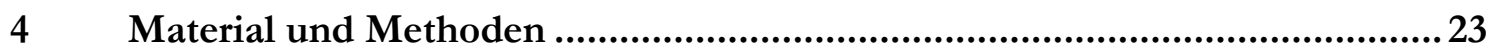

4.1 Wahl und Begründung der Methode..................................................................................23

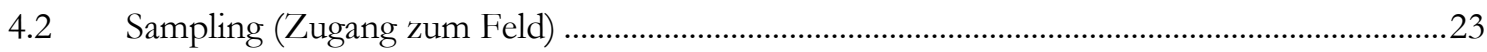

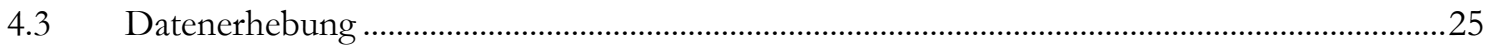

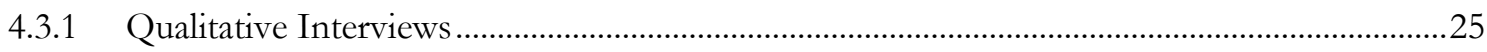

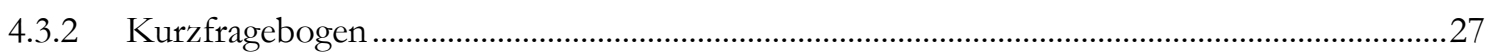

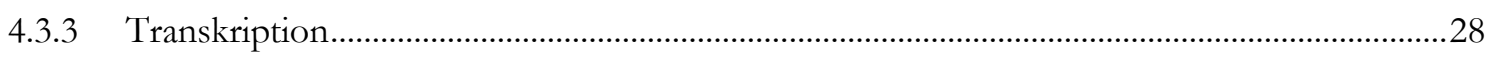

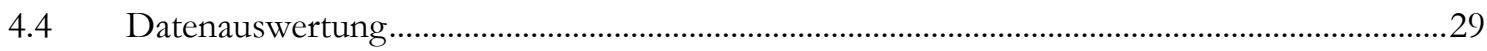

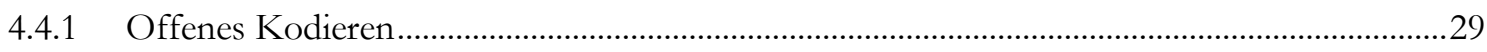

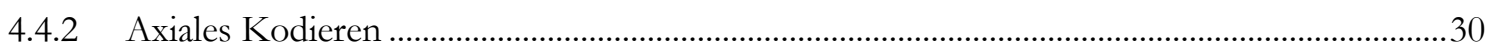

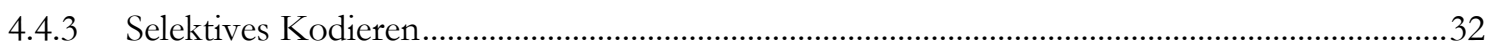

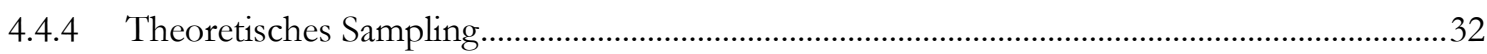

4.5 Datenschutz und Genehmigung durch die Ethikkommission.....................................................33 
$5 \quad$ Ergebnisse

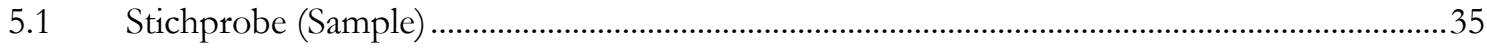

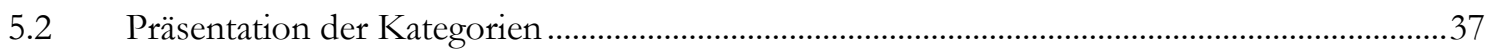

5.3 Kernkategorie: Durch KPZ entsteht „Klassengesellschaft“ auf der Station.............................38

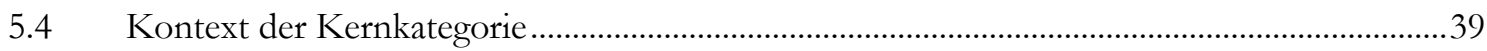

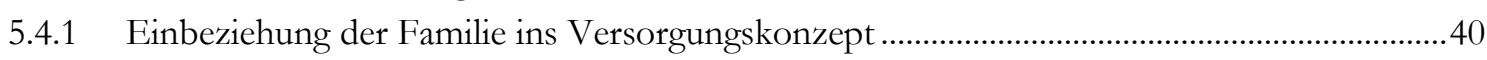

5.4.2 Aufbau UMG-eigener Versorgungsstrukturen für Palliativkinder .............................................4

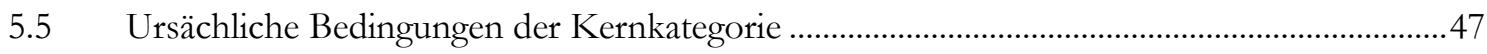

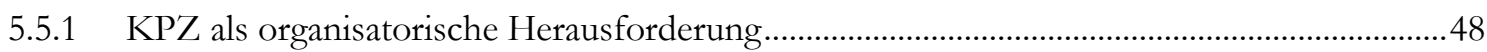

5.5.2 Abgrenzungsproblematik des Aufgaben- und Verantwortungsbereiches..................................51

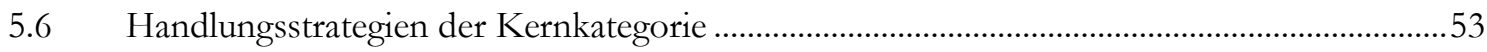

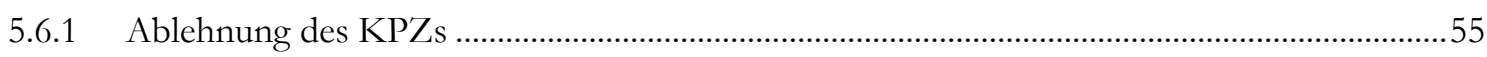

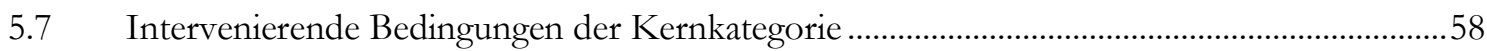

5.7.1 Sich ausgeschlossen fühlen vom Entstehungsprozess des KPZ .............................................59

5.7.2 Intra- und interprofessionelle Konflikte belasten Zusammenarbeit..........................................62

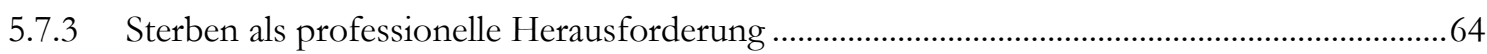

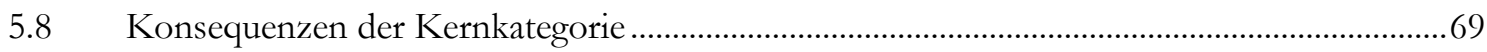

5.8.1 Überforderung und Selbstunsicherheit.............................................................................

5.8.2 KPZ als Chance zur Kompetenzerweiterung ......................................................................76

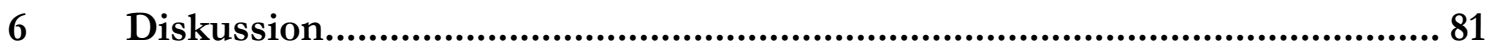

6.1 Gefühl einer entstandenen „Klassengesellschaft“ und Zwei-Klassen-Medizin .......................81

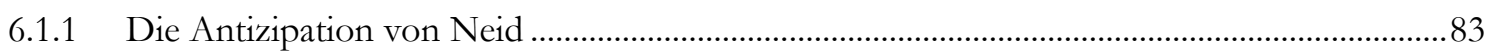

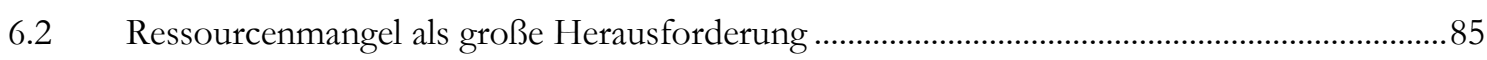

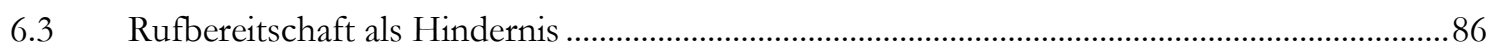

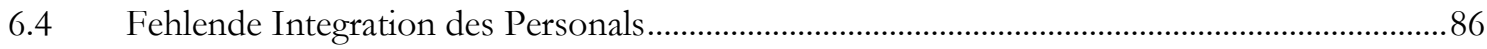

6.5 Arbeit mit Palliativkindern kann belastend sein ........................................................................ 87

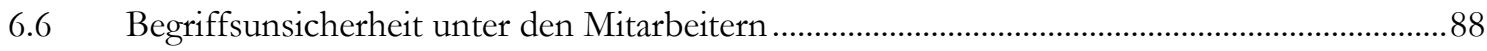

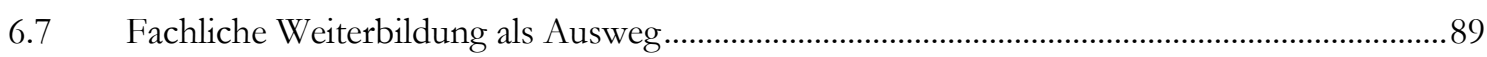

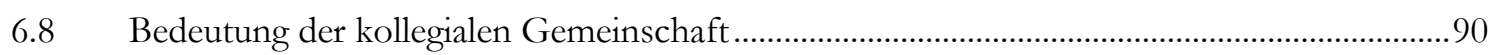

6.9 Stärken und Schwächen der Arbeit ...................................................................................

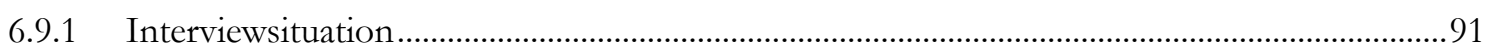

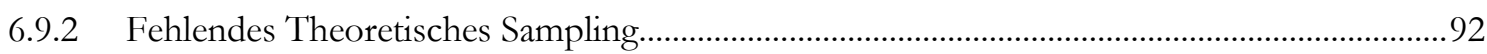

6.9.3 Besondere Stärken der Arbeit.............................................................................................

$6.10 \quad$ Fazit und Ausblick........................................................................................................ 93

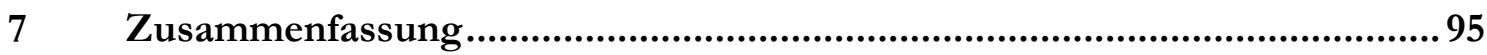

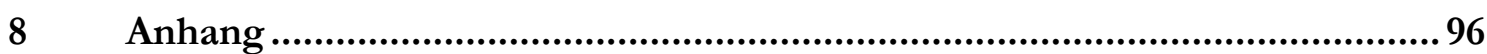

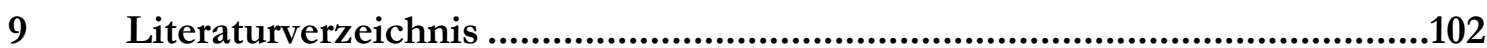




\section{Abbildungsverzeichnis}

Abbildung 1 Visualisierung des KPZs (Flyer Kinderpalliativmedizin) ............................................22

Abbildung 2 Grundriss KPZ (Flyer Kinderpalliativmedizin) ............................................................22

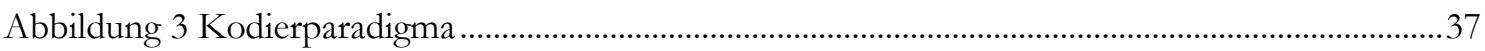

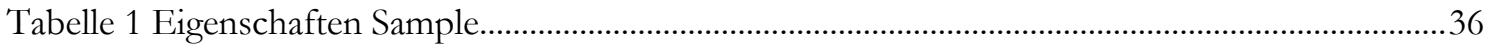




\section{Abkürzungsverzeichnis}

\begin{tabular}{|l|l|}
\hline ACT & $\begin{array}{l}\text { Association for Children with Life- } \\
\text { treatening or terminal Conditions and their } \\
\text { Families }\end{array}$ \\
\hline DGP & Deutsche Gesellschaft für Palliativmedizin \\
\hline DNR-ORDER & Do-Not-Resuscitate-Order \\
\hline GKV & Gesetzliche Krankenversicherung \\
\hline KIMBU & Kinderambulanz \\
\hline (Häusliche Kinderkrankenpflege Göttingen \\
\hline KPZ & gemeinnützige GmbH) \\
\hline LBE & Kinderpalliativzimmer \\
\hline LLE & Lebensbedrohliche Erkrankung \\
\hline PKV & Lebenslimitierende Erkrankung \\
\hline SAPV & Private Krankenversicherung \\
\hline UMG & Spezialisierte ambulante Palliativversorgung \\
\hline WHO & Universitätsmedizin Göttingen \\
\hline
\end{tabular}




\section{Vorwort}

In der Abteilung Neuropädiatrie der Klinik für Kinder- und Jugendmedizin der Universitätsmedizin Göttingen wurde im Oktober 2014 ein Palliativzimmer für Kinder und Jugendliche eröffnet, welches zur Verbesserung der stationären Versorgung schwerstkranker Kinder und Jugendlicher beitragen soll. Bei der Implementierung eines solchen Zimmers auf einer Normalstation handelt es sich in Deutschland um ein neuartiges Versorgungskonzept, das bis heute erst wenige Male realisiert wurde.

Diese Studie untersucht nicht, ob ein derartiges Versorgungskonzept eine sinnvolle Versorgungsstrukturerweiterung für Kinder und Jugendliche mit lebensbedrohlichen und lebenslimitierenden Erkrankungen darstellt. Es sollte vielmehr die Perspektive der beteiligten Mitarbeiter in den Fokus gerückt und erfahren werden, wie sie den Implementierungsprozess erlebt hatten. Die Mitarbeiterbefragungen fanden zwischen Oktober 2014 und März 2015 statt und befassten sich mit dem Planungs- und Entstehungsprozess sowie der Anlaufphase des Kinderpalliativzimmers (KPZ). Seither sind einige mit der Implementierung aufgetretene Problemfelder überdacht und verbessert worden, sodass die beschriebenen Umstände die Entstehungsphase und nicht die aktuelle Situation (September 2016) beschreiben.

Die Ergebnisse dieser Studie sollen andere Kliniken, die ein derartiges Versorgungsmodell realisieren wollen, unterstützen und zeigen, welch große Bedeutung die Mitarbeiterperspektive und das Einbeziehen der Mitarbeiter schon in den Planungsprozess für die erfolgreiche Entstehung eines Kinderpalliativzimmers und die Arbeit in einem solchen Zimmer haben. 


\section{$2 \quad$ Einleitung}

Die Sterblichkeit im Kindesalter ist in den letzten 60 Jahren deutlich gesunken. Verstarben 1955 noch 48614 Kinder in der Bundesrepublik Deutschland innerhalb des ersten Lebensjahres, so waren es 2014 nur noch 2284 Kinder (Statistisches Bundesamt Todesursachen in Deutschland 2014). Trotz sinkender Sterblichkeit im Kindesalter leiden in der Bundesrepublik Deutschland Schätzungen zufolge ca. 22000 Kinder und Jugendliche an lebensbedrohlichen (LBE) und lebenslimitierenden (LLE) Erkrankungen. Jedes Jahr versterben etwa 3000 Kinder, Jugendliche und junge Erwachsene an ihrer Krankheit (Zernikow und Henkel 2009). Für LBE, wie beispielsweise Krebserkrankungen, existieren potentiell kurative Therapien, deren Versagen aber durchaus wahrscheinlich ist. Bei den unter 15-Jährigen erkrankte 2014 in der Bundesrepublik Deutschland 1 von 1200 Kindern an Krebs. Es gab ca. 400 Todesfälle von Kindern, die auf eine onkologische Erkrankung zurückzuführen waren. Mit ca. 30\% zählen Leukämien zu den häufigsten onkologischen Krankheitsbildern - zum Vergleich bei Erwachsenen sind Leukämien die elfthäufigste Krebsform. Diese meist akuten Leukämieformen haben eine gute Prognose und 89\% der erkrankten Kinder überleben mindestens 15 Jahre Jahresbericht 2015 Deutsches Kinderkrebsregister). Für LLE, wie beispielweise Muskeldystrophien oder die Bronchopulmonale Dysplasie, gibt es keine realistische Heilungschance. Eine Erkrankung erfolgt vor dem 18. Lebensjahr, und die Kinder und jungen Erwachsenen versterben meist vor Erreichen ihres 40. Lebensjahres (Zernikow und Nauck 2008). In Deutschland existieren keine soliden epidemiologischen Erhebungen zu LBE und LLE, da Register zur Erfassung fehlen. Lediglich Krebserkrankungen werden im Deutschen Kinderkrebsregister aufgenommen. Die LBE und LLE können in die international gebräuchlichen vier Gruppen nach der Association for Children with Life-Threatening or Teminal Conditions and their Families (ACT) unterteilt werden (ACT 2009). Mithilfe dieser Einteilung lassen sich Krankheitsverläufe einschätzen und der voraussichtliche Versorgungsbedarf für das erkrankte Kind ermessen:

In der ersten Gruppe werden LBE zusammengefasst, für die potentiell kurative Therapien existieren, deren Scheitern jedoch wahrscheinlich ist. Dazu zählen Krebserkrankungen, aber auch irreversibles Organversagen z. B. des Herzens. 
In der zweiten Gruppe ist die Lebenserwartung reduziert und intensive Therapien zur Lebensverlängerung sind vonnöten. Zu dieser Gruppe zählen beispielsweise die Zystische Fibrose oder Muskeldystrophien.

In die dritte Gruppe werden progressive Erkrankungen, wie z. B. die Mukopolysaccharidosen, eingeordnet. Hier existieren keine kurativen Optionen und ausschließlich palliative Therapien können durchgeführt werden.

In die vierte Gruppe lassen sich schwere Beeinträchtigungen, meist neurologischer Ursache, einordnen. Sie gehen häufig mit Komplikationen einher, die zu unvorhersehbaren Verschlechterungen führen können. Trotz dieser Komplikationen haben sie keinen progressiven Verlauf. Erkrankungen, die zu dieser Gruppe gehören, sind z. B. schwere Mehrfachbehinderungen aufgrund von Hirn- oder Rückenmarksverletzungen oder die Bronchopulmonale Dysplasie (ACT 2009). 


\section{Stand der Forschung}

\subsection{Palliativmedizin im Kindesalter}

Kinder und Jugendliche, die nach dem Schema der ACT einer der vier Gruppen zugeteilt werden können, benötigen laut Definition der World Health Organization (WHO) für Palliativmedizin im Kindesalter eine aktive palliativmedizinische Betreuung, die die physischen, psychischen und spirituellen Bedürfnisse des Kindes beachtet. Neben der Betreuung des erkrankten Kindes ist auch die Versorgung der Familie besonders wichtig (WHO Definition of Palliative Care). Zur „Familie“ gehören in diesem Fall Eltern und Geschwister des Kindes, aber auch Großeltern, Verwandte und Freunde, die das soziale Umfeld des Kindes und seiner Eltern darstellen (Nolte-Buchholtz und Janisch 2015). Für eine effektive Palliativversorgung wird ein multidisziplinäres Team aus Kinderärzten ${ }^{1}$, Palliativmedizinern, Psychologen, Seelsorgern u.v.m. benötigt, das die Familie ins Betreuungskonzept einschließt und regionale Unterstützungsangebote, z. B. Selbsthilfegruppen, zugänglich macht. Diese Betreuung sollte bereits bei Diagnosestellung beginnen (WHO Definition of Palliative Care). Viele der LBE und LLE verlaufen nicht unmittelbar tödlich (vgl. Kapitel 2 Einleitung). Jedoch sollten sich - wie auch von der WHO gefordert - lebensverlängernde und auf Heilung ausgerichtete Therapien und eine Unterstützung der physischen, psychischen und spirituellen Bedürfnisse nicht in einer Entweder-oder-Entscheidung gegenüberstehen, sondern von Anfang an Hand-in-Hand gehen und sich ergänzen (Sahler et al. 2000).

\subsubsection{Sterblichkeit von Kindern - Häufigkeit in verschiedenen Altersgruppen}

Nach Angaben des Statistischen Bundesamtes verstarben 2014 ca. 4000 Kinder und Jugendliche bis zum 20. Lebensjahr². Von diesen Kindern und Jugendlichen verstarben ca. $60 \%$ innerhalb ihres 1 . Lebensjahres und davon 70\% innerhalb der ersten 28 Lebenstage (Statistisches Bundesamt Todesursachen in Deutschland 2014). Unter allen Todesfällen von Kindern und Jugendlichen stellen Neugeborene somit die größte Gruppe dar. In einer

\footnotetext{
${ }^{1}$ In dieser Arbeit werden zu Erleichterung der Lesbarkeit in der Einzahl immer beide Geschlechter und in der Mehrzahl nur das männliche Geschlecht genannt. Es sei aber darauf hingewiesen, dass immer beide Geschlechter gemeint sind.

${ }^{2}$ Diese Zahl betrifft alle Todesfälle der unter 20-Jährigen und ist nicht auf solche beschränkt, die auf LBE und LLE zurückzuführen sind. Darin eingeschlossen sind z. B. auch Todesfälle durch Verletzungen, Vergiftungen oder äußere Ursachen von Morbidität und Mortalität.
} 
Studie, welche die Todesumstände hospitalisierter Kinder in Nashville (Tennessee, USA) untersuchte, zeigte sich ebenfalls, dass die meisten Kinder innerhalb ihres ersten Lebensjahres verstarben. Bei den 105 untersuchten Todesfällen betrug das durchschnittliche Lebensalter bei Todeseintritt lediglich 68 Tage (Carter et al. 2004). Zu ähnlichen Ergebnissen kam eine Studie, die 13761 Todesfälle von Kindern in 60 verschiedenen Krankenhäusern in den Jahren 1991, 1994 und 1997 in den USA untersuchte. Hier verstarben $60 \%$ der Kinder innerhalb ihres ersten Lebensjahres, 48\% davon sogar innerhalb der ersten 28 Lebenstage (Feudtner et al. 2002). Ursächlich für diesen frühen Tod sind meist perinatale Komplikationen, angeborene Fehlbildungen, Infektionen und andere Ursachen, die eine intensivmedizinische Versorgung notwendig machen (Sahler et al. 2000). Auch diesen Kindern und ihren Familien sollte eine palliativmedizinische Versorgung zugänglich gemacht werden. Abhängig vom Zeitpunkt der Diagnosestellung kann damit pränatal, unmittelbar nach der Geburt oder während der Neonatalperiode begonnen werden (Garten 2014). Jenseits des ersten Lebensjahres stellen Krebserkrankungen die häufigste Todesursache dar. $\mathrm{Zu}$ diesem Ergebnis kam eine amerikanische Studie, die Todesursachen von Kindern und jungen Erwachsenen in den USA über einen Zeitraum von 20 Jahren untersuchte (Feudtner et al. 2001).

\subsubsection{Symptome in der Kinderpalliativmedizin}

Von den Versorgenden der erkrankten Kinder wird gefordert, dass sie ab dem Moment der Diagnosestellung die physischen und psychosozialen Leiden des Kindes erkennen und sie im Rahmen einer suffizienten Symptomlinderung medikamentös oder mithilfe geeigneter nichtmedikamentöser Therapiemethoden behandeln. Aufgrund der vielen verschiedenen Erkrankungen, die die palliativmedizinische Versorgung eines Kindes erforderlich machen können, liegt bei den Betroffenen eine Vielzahl unterschiedlicher Symptome vor. Zu den häufigsten Symptomen am Lebensende gehören Schmerzen, Atemnot, Müdigkeit und Angst (Wolfe et al. 2000; Führer 2011). Das am häufigsten behandelte Symptom stellt mit $76 \%$ der Schmerz dar. Wolfe et al. (2000) konnten jedoch zeigen, dass die Behandlung nur in $27 \%$ der Fälle erfolgreich war. Ähnliche Ergebnisse ergaben sich auch beim Symptom Atemnot. Eine niederländische Studie konnte bei der erfolgreichen Schmerzbehandlung deutlich bessere Ergebnisse erzielen. Hier kam es in über 70\% der Fälle zu einer teilweisen oder völligen Auflösung der Schmerzsymptomatik nach medizinischem Eingreifen (Theunissen et al. 2007). Wolfe et al. (2000) konnten ebenfalls zeigen, dass die Einschätzung der Symptome des Kindes zwischen Eltern und behandelnden Ärzten teils stark voneinander abwichen. Das Kind ist im Laufe der Erkrankung vielen Belastungen 
ausgesetzt. So stellt die Krankheit und die damit verbundenen Einschränkungen eine Störung der gewohnten Alltagsroutine dar, z. B. kommt es durch die Hospitalisation zur Trennung von den Eltern und dem gewohnten sozialen Umfeld. Dies löst zusammen mit den physischen Symptomen der Krankheit, der eingeschränkten Aktivität und dem Verlust der autonomen Kontrolle über den Körper beim Kind Ängste aus (Bürgin 1978). Theunissen et al. (2007) identifizierten in ihrer Studie Traurigkeit, Schwierigkeiten mit den Eltern über ihre Gefühle bezüglich Krankheit und Tod zu sprechen, Angst vor dem Alleinsein, Perspektivverlust und Unabhängigkeitsverlust als die fünf häufigsten psychosozialen Symptome von Kindern am Lebensende. Den Eltern zufolge wurde grade diesen Symptomen zu wenig Beachtung von professioneller Seite geschenkt, da sich das medizinische Personal vor allem auf die physischen Symptome und deren Linderung konzentrierte.

\subsection{Das Versorgerteam in der Kinderpalliativmedizin}

An der Behandlung lebensbedrohlich oder lebenslimitierend erkrankter Kinder ist ein multidisziplinäres Team beteiligt, welches seine Aufmerksamkeit den physischen, psychosozialen und spirituellen Bedürfnissen des Kindes widmet und auch dessen Familie unterstützen sollte (vgl. 3.1 Palliativmedizin im Kindesalter).

\subsubsection{Multiprofessionelle Zusammenarbeit}

Für eine hochwertige Patientenversorgung ist die Qualität der interprofessionellen Zusammenarbeit von besonderer Bedeutung. Die medizinische Versorgung des Kindes wird vornehmlich von Ärzten sowie Gesundheits- und Kinderkrankenpflegern geleistet. Die Zusammenarbeit dieser beiden Berufsgruppen war Thema einer Untersuchung von Schmalenberg und Kramer (2009). Sie unterschieden fünf verschiedene Beziehungsverhältnisse, die nebeneinander in einem Krankenhaus existieren können. Sie beschreiben drei Modelle, eine gute Arbeitsbeziehung zwischen Ärzteschaft und Pflegepersonal und zwar die „kollegiale Beziehung“, die „kollaborative Beziehung“ und die „Student-Lehrer-Beziehung“. Die beiden erstgenannten sind geprägt von gegenseitigem Vertrauen und Respekt, jedoch ist die Ärztin oder der Arzt dem Pflegepersonal in der „kollaborativen Beziehung“ übergeordnet. In der „Student-Lehrer-Beziehung“ kann sowohl das Mitglied der Ärzteschaft als auch das Mitglied des Pflegepersonals die Lehrerrolle einnehmen. Das Pflegepersonal kann sich z. B. in die Lehrerrolle einfügen, wenn die Ärztin oder der Arzt über wenig Berufserfahrung verfügt oder fremd im 
Fachgebiet ist. Als neutral einzustufen ist die „Freundlicher-Fremder-Beziehung“, bei der es zu einem sachlichen Austausch von Informationen kommt. Als ungünstig erweist sich die fünfte Beziehungsart, die von feindseligem und gegensätzlichem Verhalten geprägt ist. Unzufriedenheit, Ärger und gegenseitige Vorwürfe spielen hier eine entscheidende Rolle. Besteht diese Art von Beziehung, ist eine optimale Patientenversorgung nur schwer zu realisieren, weil nicht miteinander, sondern gegeneinander gearbeitet wird (Schmalenberg und Kramer 2009).

Die Zusammenarbeit und Kommunikation zwischen den beiden Professionen (Ärzteschaft und Pflegepersonal) wurde auch in einer amerikanischen Studie untersucht. Zwischen Ärzten und Pflegenden bestand ein hierarchisches Verhältnis zugunsten der Ärzte. Beide Gruppen maßen sich große Bedeutung für eine gute Patientenversorgung zu und fühlten sich von der jeweils anderen Partei dafür häufig nicht ausreichend wertgeschätzt. Diese fehlende Wertschätzung und der Verweis auf starre hierarchische Strukturen wurden besonders von den Pflegenden moniert. Eine interprofessionelle Kommunikation ist wichtig, um Fehlern und Missverständnissen in der Patientenversorgung vorzubeugen. In diesem Bereich sahen viele Studienteilnehmer Verbesserungsbedarf (Lancaster et al. 2015).

\subsubsection{Emotionale Belastung der Versorgerinnen und Versorger}

Die Arbeit mit lebensbedrohlich und lebenslimitierend erkrankten Kindern stellt für die Behandler eine Herausforderung dar. In einem auf Heilung ausgerichteten System, in dem Kinderärzte mit wenigen sterbenden Patienten konfrontiert werden - ein Allgemeinpädiater oder eine Allgemeinpädiaterin in Nordamerika versorgt weniger als drei sterbende Kinder pro Jahr - kann ein sterbendes Kind ein Gefühl von Schuld verursachen, weil es nicht vor dem Tod bewahrt werden konnte (Sahler et al. 2000). In ihrer Ausbildung bekommt das medizinische Personal vermittelt, dass Heilung des Patienten und Lösung des medizinischen Problems oberste Handlungsziele sind. Ein Scheitern an diesen Zielen und der Verlust eines Patienten werden somit häufig als persönliche Niederlage wahrgenommen. Durch die gesellschaftlichen Ansprüche und die fehlende Vorbereitung in der medizinischen Ausbildung fühlen sich viele Ärzte unvorbereitet und überfordert. Dies betrifft oft auch die Kommunikation mit Patienten und Angehörigen in Ausnahmesituationen (Husebø 2009; Zernikow und Henkel 2009). Eine Studie der Universität Stanford befragte zu diesem Thema Krankenhauspersonal einer Kinderklinik und bestätigte, dass sich viele Mitarbeiter unvorbereitet und unerfahren fühlten. Dies betraf vor allem die Kommunikation mit den Patienten und Angehörigen bezüglich Fragen am Lebensende, Gespräche über den Wunsch nach Wiederbelebung des Kindes im Falle von 
Kreislaufversagen und den Übergang des Kindes in eine rein palliative Versorgung. Mehr als die Hälfte der befragten Mitarbeiter fühlten sich auch bei der Gewährleistung einer effektiven Schmerzkontrolle unerfahren. Diese fehlende Erfahrung und Unsicherheit verursachte Traurigkeit und emotionale Belastung, was bis zu Depressionen und Burnout führen kann (Contro et al. 2004). Eine britische Studie untersuchte, welche Faktoren Stress und Befriedigung im Job auslösten und verglich dabei Palliativmediziner mit Ärzten anderer Fachdisziplinen. Es fühlten sich lediglich 7\% der Palliativmediziner unzureichend ausgebildet für eine effektive Symptomkontrolle und 35\% für die Kommunikation mit Patienten und Angehörigen. Ärzte anderer Fachdisziplinen sahen hier größere Defizite. Die Palliativmediziner empfanden auch weniger Stress bei Aufgaben wie dem Umgang mit LBE und LLE und dem Tod von Patienten oder der Überbringung schlechter Nachrichten als ihre Kollegen (Graham et al. 1996). Daraus ist abzuleiten, wie bedeutend die Erfahrung im Umgang mit Sterben und Tod ist und wie Stress und emotionale Überlastung dadurch reduziert werden können.

Auch für das Pflegepersonal stellt der Tod eines Kindes eine große emotionale Belastung und dessen Verarbeitung eine große Herausforderung dar. So erhielten Contro et al. (2004) auf ihre Frage nach der schwierigsten Erfahrung im Zusammenhang mit dem Tod eines Kindes von den Pflegenden die Antwort, dass der persönliche Schmerz und die fehlende Unterstützung bei dessen Verarbeitung am schwierigsten seien. Dabei erfuhren laut eigenen Angaben 54\% der Befragten keine Unterstützung durch ihren Arbeitgeber oder ihre Arbeitgeberin, sondern mussten selbst Wege finden, den Tod des Patienten zu verarbeiten, z. B. indem sie auf dem Weg nach Hause weinten oder Alkohol tranken. Einige berichteten sogar, dass es ihnen schwerfiele, ihre Aufgaben auf der Station zu erledigen, bis sie den Tod des Kindes nicht z. B. durch Gespräche mit Kollegen anfangen konnten zu verarbeiten.

\subsection{Unterschiede in der Palliativversorgung von Erwachsenen und Kindern}

Es bestehen Unterschiede in der palliativmedizinischen Versorgung von Kindern, Jugendlichen und jungen Erwachsenen zu der älterer Erwachsener: Bei älteren Erwachsenen stellen bösartige Neubildungen mit 86,8\% die Hauptdiagnose dar, die eine palliativmedizinische Versorgung notwendig machen (Hospiz- und Palliativerhebung 2010). Bei Kindern und Jugendlichen ist die Gruppe der Grunderkrankungen deutlicher heterogener (vgl. Kapitel 2 Einleitung). Aufgrund dessen unterscheiden sich auch die hauptsächlich vorliegenden belastenden Symptome der Patienten. Die 
Krankheitsprognosen bei Kindern und Jugendlichen unterscheiden sich bei den verschiedenen Erkrankungen und die Krankheitsverläufe sind oft schwer einzuschätzen (vgl. Kapitel 2 Einleitung). Die palliative Versorgung erstreckt sich oft über Jahre. Bei älteren Erwachsenen hingegen wird häufig nur über Wochen und Monate eine palliativmedizinische Versorgung in Anspruch genommen. Eine große Herausforderung für die Behandler stellt die umfassende Versorgung der ganzen Familie in der palliativmedizinischen Versorgung von Kindern und Jugendlichen dar. Die Familien übernehmen die Versorgung ihres Kindes meist über viele Jahre und sind dadurch stark belastet. Auch in der palliativmedizinischen Betreuung älterer Erwachsener ist die psychosoziale Begleitung der Familie von Bedeutung, doch nimmt sie einen kleineren Stellenwert ein. In speziellen Kinderhospizen, in denen vorrangig Entlastungspflege geleistet wird, können Patienten und Familien neue Kraft schöpfen. Dies ist ein großer Unterschied zu Hospizen für Erwachsene, in denen $>90 \%$ der aufgenommenen Patienten versterben. Die ambulante Palliativversorgung für Kinder ist längst nicht so flächendeckend vorhanden, wie dies für ältere Erwachsene der Fall ist, was mit der geringeren Zahl von Betroffenen zusammenhängt (Zernikow und Nauck 2008).

\subsection{Die Situation der Familie des erkrankten Kindes}

\subsubsection{Emotionale Belastung von Eltern und Geschwistern}

Die unheilbare Erkrankung eines Kindes und dessen Tod stellen für die Familie des Kindes eine große emotionale Belastung dar (Rando 1985; James und Johnson 1997). Die Trauer nach dem Tod eines Kindes dauert oft Jahre an und ist teils mit Schuldgefühlen verbunden, weil man das eigene Kind nicht vor Leid und Tod schützen konnte. Dabei scheinen Schuldgefühle vor allem bei den Eltern zu bestehen, die die medizinische Versorgung ihres Kindes als unzureichend empfunden haben (Surkan et al. 2006). Neben den Eltern der erkrankten Kinder muss auch - ggf. vorhandenen - gesunden Geschwisterkindern besondere Beachtung geschenkt werden. Ihre Trauer wird oft wenig zur Kenntnis genommen, weil oft die Eltern im Fokus der Aufmerksamkeit stehen. Eine amerikanische Studie befragte zu diesem Thema Eltern und gesunde Geschwister von an Krebs erkrankten Kindern zur Reaktion der Geschwister auf die Krebserkrankung des Bruders oder der Schwester. Es zeigten sich Kommunikationsprobleme und eine unterschiedliche Wahrnehmung der Situation zwischen Eltern und gesunden Geschwisterkindern. In Fällen großer Wahrnehmungsdiskrepanz und fehlender Kommunikation waren negative Verhaltensänderungen des gesunden Kindes die Folge (Breyer et al. 1993). Um dem 
entgegenzuwirken, sollten Eltern ihre gesunden Kinder nicht über der Krankheit des betroffenen Kindes vergessen und auch ihnen Aufmerksamkeit und Zuwendung schenken. Um Geschwisterkinder aufzufangen und Eltern zu unterstützen, gibt es an verschiedenen Stellen Angebote, die sich speziell an deren Bedürfnisse richten. So bieten viele Kinderkliniken, aber auch beispielsweise die Deutsche Kinderkrebsstiftung, Beratungs- und Betreuungsangebote für Geschwister an. Der Tod des Bruders oder der Schwester stellt für die überlebenden Geschwister eine große Belastung dar. Es bestehen z. T. Schuld- und Verantwortungsgefühle für die Erkrankung oder den Tod des Bruders oder der Schwester. Der Umgang mit dieser Schuld kann sich, abhängig von der Persönlichkeit des Kindes, unterschiedlich äußern, z. B. in Form eines depressiven Zurückziehens oder durch auffälliges Verhalten in der Schule (Cain et al. 1964). Aber auch Eltern können einen negativen Einfluss auf die Trauer ihres überlebenden Kindes nehmen, indem sie z. B. das verstorbene Kind idealisieren und das Geschwisterkind mit diesem Idealbild vergleichen (Brent 1983) oder überfürsorglich werden aus Angst, ein weiteres Kind zu verlieren (Cain et al. 1964; Brent 1983). Es können sich jedoch auch positive Entwicklungen und Verhaltensänderungen bei den Geschwisterkindern zeigen, wie z. B. wachsende Sensibilität und Empathie und eine raschere Persönlichkeitsentwicklung (Williams 1997; Murray 1999).

\subsubsection{Die Bedeutung der Beziehung zwischen der Familie und den Behandlern}

Im Verlauf einer lang andauernden Erkrankung entwickelt sich eine Beziehung zwischen der Familie des betroffenen Kindes und dessen Behandlern. Es zeigt sich, dass die Kommunikation zwischen Familie und Ärztin oder Arzt einen großen Einfluss auf die Gefühle und den Trauerprozess der Familie hat. So befragten Bright et al. (2009) Eltern und pflegende Angehörige, deren Kind verstorben war, wie sie die Interaktion mit der verantwortlichen Ärztin oder dem Arzt während der Behandlung und nach dem Tod des Kindes erlebt hatten. Dabei interessierte man sich besonders für Kommunikationsprobleme zwischen Angehörigen und Ärzten, welche Unterstützungseinrichtungen der Arzt oder die Ärztin den Angehörigen zugänglich machen konnte und wie der Kontakt über den Tod des Kindes hinaus aussah. Die Befragten verloren ihre Kinder nicht nur durch chronische Erkrankungen, sondern auch plötzlich und unerwartet infolge von z. B. Verkehrsunfällen. Kritisiert wurde von den betroffenen Angehörigen die oft harte und sachliche Ausdrucksweise der Ärzte und die Verwendung von Phrasen wie „Ihr Kind ist nun an einem besseren Ort“.

Viele Eltern hätten sich unterstützende Maßnahmen vom behandelnden Arzt oder der Ärztin gewünscht. Dazu zählten etwa das Verweisen an Selbsthilfegruppen oder, dass jener 
bzw. jene den Kontakt zur Familie nicht sofort abreißen lässt, sondern ihn beispielsweise in Form von späteren Anrufen oder schriftlicher Kommunikation aufrechterhält. Auch Contro et al. (2004) beschäftigten sich mit den Bedürfnissen trauernder Eltern. Viele Familien wünschen sich auch nach dem Tod ihres Kindes Kontakt zu den Behandlern. Durch die oft lange Krankheit und den damit verbundenen intensiven Kontakt mit dem Krankenhausteam wurde dieses z. T. als zweite Familie wahrgenommen. Brach der Kontakt nach dem Tod des Kindes abrupt ab, stellte dies einen zusätzlichen Verlust dar. Es gibt keine allgemeingültige Definition, was genau unter einer Aufrechterhaltung des Kontakts zwischen der Familie des verstorbenen Kindes und den an der Versorgung beteiligten Mitarbeitern verstanden wird. Einige Krankenhäuser haben Unterstützungsangebote für trauernde Familien, die auch dafür sorgen sollen, dass der Kontakt nicht mit dem Tod des Kindes beendet wird. Dazu gehört z. B. das Verschicken von Karten, Telefonanrufe oder die Initiierung von Selbsthilfegruppen für betroffene Eltern. Doch handelt es sich hierbei häufig um institutionalisierte Angebote, die nicht mit einem Kontakt mit den tatsächlichen Behandlern gleichzusetzen sind. Eine amerikanische Studie befragte zu diesem Thema pädiatrische Intensivmediziner, inwieweit sie den Kontakt mit trauernden Familien aufrechterhalten würden. Sie kamen zu dem Ergebnis, dass $87 \%$ der Ärzte die Familie manchmal oder immer kontaktierten. Darin eingeschlossen waren jedoch sowohl eine aktive als auch eine passive Form des Kontakts. Unter einer aktiven Kontaktpflege wurden hier Telefonanrufe, Briefe und Treffen verstanden, wohingegen die passive Kontaktpflege, von welcher die meisten Ärzte berichteten, eine Weitergabe der persönlichen Kontaktdaten und ansonsten eine Delegation dieser Aufgabe an andere Mitarbeiter des Krankenhauses bedeutete. Zwei Drittel der Befragten nahmen auch nur maximal in einem Viertel der Todesfälle an der Beerdigung teil. Als Gründe für dieses häufige Fernbleiben wurden vor allem Zeitmangel und logistische Probleme, aber auch ein Unwohlsein in dieser emotionalen Situation genannt. Es gab auch Behandler, die die Teilnahme als unpassend und aufdringlich empfanden und deshalb von Beerdigungen fernblieben. Dabei profitieren nicht nur Familien von diesem bestehenden Kontakt, sondern auch den Ärzten kann es helfen, den Tod des Patienten zu verarbeiten (Borasino et al. 2008). Zu diesem Ergebnis kam auch Holland (2002).

Der Informationsaustausch zwischen Behandlern und dem Kind und seiner Familie ist von großer Bedeutung. Das Gespräch über eine LBE oder LLE und über den Tod stellt für alle Beteiligten eine große emotionale Belastung dar. In der Kinderpalliativmedizin hat die Ärztin oder der Arzt zwei Gesprächspartner: Zum einen das erkrankte Kind und zum anderen die Eltern, die - abhängig vom Alter und der geistigen Entwicklung des Kindes - 
als primäre Gesprächspartner fungieren. Diese Dreieckskonstellation kann schwierig sein, wenn die Eltern ihrem Kind beispielsweise Informationen über die Schwere und das Ausmaß der Krankheit vorenthalten, weil sie es beschützen wollen. In solch einem Fall sollten die Behandler offene Gespräche mit den Eltern führen und sich auf ein gemeinsames Vorgehen einigen. Es ist nicht empfehlenswert, als Behandler oder Behandlerin gegen den Willen der Eltern ein offenes Gespräch mit dem Kind über dessen nahenden Tod zu führen. Daraus kann sich für das Kind ein belastender Loyalitätskonflikt entwickeln (Zernikow und Henkel 2009). Eine schwedische Studie kam zu dem Ergebnis, dass alle Eltern, die offene Gespräche mit ihrem Kind über dessen Tod geführt hatten, diese Entscheidung im Nachhinein nicht bereuten. Von den Eltern, die ein Gespräch mit ihrem Kind über den Tod vermieden hatten, bereuten diese Entscheidung ca. 30\% (Kreicbergs et al. 2004). Abgesehen von der Frage, ob Eltern mit ihrem Kind offen über dessen Tod sprechen, zeigt sich in der Literatur, dass es vielen Eltern wichtig ist, in den Entscheidungsprozess bezüglich Fragen am Lebensende integriert zu sein. Dies hat großen Einfluss auf ihre Trauer und die Verarbeitung des Todes. Es ist deshalb wichtig, dass die Behandler mit den Eltern ausführliche Gespräche führen. Besondere Angst hatten Eltern davor, dass ihr Kind am Lebensende leiden muss (Vos et al. 2015). Es hat deshalb besondere Bedeutung, dass das Behandlerteam den Eltern diese Angst nehmen kann.

\subsubsection{Vorausplanung der Versorgung}

Bei Kindern mit LBE und LLE muss zusammen mit den Eltern überlegt werden, ob kardiopulmonale Reanimation und andere belastende Behandlungsmaßnahmen in jedem Stadium der Erkrankung im Interesse des Kindes sind, oder ob sie ab einem bestimmten Zeitpunkt unterlassen werden sollten (Morrison und Berkowitz 2007). Zu diesem Zwecke kann es sinnvoll sein, dass die Wünsche des Patienten in einer Empfehlung zum Vorgehen in Notfallsituationen - in einer sogenannten Do-Not-Resuscitate-Order (DNR-Order) festgehalten werden. Dort wird dokumentiert, welche Maßnahmen das Kind bzw. seine Sorgeberechtigten für angemessen halten und welche in einer Notfallsituation unterlassen werden sollen. Diese Verschriftlichung gibt Ersthelfern, welche mit der Krankengeschichte des Kindes nicht genau vertraut sind, eine Handlungssicherheit in Notfallsituationen (Rellensmann und Hasan 2009). In einer kanadischen Studie wurde untersucht, ob eine DNR-Order verfasst wurde und mit welchem zeitlichen Abstand zum eintretenden Tod des Kindes dies geschah. Von den untersuchten Fällen hatten sechs Kinder bereits vor ihrem letzten Krankenhausaufenthalt eine DNR-Order. Bei 79\% der verbleibenden Kinder wurde im Verlauf der letzten Hospitalisation eine DNR-Order verfasst, wohingegen in den 
restlichen untersuchten Todesfällen im Vorhinein kein Gespräch über gewünschte medizinische Maßnahmen in Notfallsituationen am Lebensende geführt wurde. Diese Kinder starben dann in Folge einer gescheiterten kardiopulmonalen Wiederbelebung. Auffällig war, dass die mittlere Dauer zwischen dem Gespräch der Ärztin oder des Arztes mit den Eltern und dem Kind, dem Festhalten ihrer Wünsche in einer DNR-Order und dem Tod des Kindes im Mittel weniger als 1 Tag lag (McCallum et al. 2000). Eine amerikanische Studie kam - mit einer mittleren verbleibenden Zeit von 2,3 Tagen zwischen Verfassung der DNR-Order und Eintritt des Todes - zu vergleichbaren Ergebnissen (Lantos et al. 1993). Als Gründe für diese oft späte Verfassung einer solchen Weisung werden angeführt, dass der Tod eines Kindes und Gespräche darüber für alle Beteiligten emotional belastend sind und deshalb hinausgezögert oder sogar gänzlich vermieden werden (Dominica 1987; McCallum et al. 2000). Lantos et al. (1993) kamen auch zu dem Ergebnis, dass im Falle der Verfassung einer DNR-Order in 92\% der Fälle die Initiative von den Ärzten und nicht vom Kind oder den Eltern ausging.

\subsubsection{Sterbeorte für Kinder}

Neben der Entscheidung, welche medizinischen Maßnahmen am Lebensende ergriffen werden sollen, spielt auch der Ort, an dem das Kind sein Lebensende verbringt und verstirbt, für die Familien eine wichtige Rolle. Eltern und Kinder haben innerhalb des Gesundheitssystems meist drei Alternativen für den Ort der Pflege am Lebensende und zum Sterben: Das Zuhause, das Krankenhaus (Kinderstation oder Intensivstation) und das Kinderhospiz. Für viele Eltern ist die Planung des letzten Pflegeplatzes und des Ortes zum Sterben besonders wichtig, weil es das Gefühl vermittelt, vorbereitet zu sein. Von den in einer Studie befragten Eltern konnten 62\% den Sterbeort ihres Kindes planen und in 97\% der Fälle konnten diese Pläne auch umgesetzt werden (Dussel et al. 2009). Es zeigt sich, dass viele Eltern es bevorzugen, dass ihr Kind in der letzten Phase seines Lebens zu Hause ist und dort im Kreise seiner Familie verstirbt (Vickers et al. 2007) und dass diese Präferenz zunimmt (Feudtner et al. 2007). Neben den Eltern bevorzugten auch die Behandler für die Pflege am Lebensende des Kindes eine Versorgung zu Hause. Falls dies nicht möglich sein sollte, z. B. wegen nicht handhabbarer Symptome, wird das Krankenhaus - vor dem Hospiz - an zweiter Stelle gewählt (Kassam et al. 2014). Abweichende Ergebnisse ergab eine kanadische Studie. Hier zeigte sich eine gleichmäßige Verteilung zwischen Zuhause, Krankenhaus und Hospiz als präferierten Ort für das Versterben des Kindes (Siden et al. 2008). Hierbei muss jedoch bedacht werden, dass nicht alle Versorgungsmöglichkeiten überall in gleichem Maße zur Verfügung stehen. 
Siden et al. (2008) konnten außerdem zeigen, dass die Diagnose einen Einfluss auf den Sterbeort des Kindes hat. Es wurde unterschieden zwischen Patienten mit Krebserkrankungen und nicht an Krebs erkrankten Patienten. Kinder mit einer onkologischen Erkrankung starben bevorzugt zu Hause, wohingegen nicht an Krebs erkrankte Patienten, dazu zählten z. B. Kinder mit kardiopulmonalen oder neuromuskuläre Erkrankungen und chromosomalen Syndromen, bevorzugt im Krankenhaus verstarben. Falls der Tod im Krankenhaus unumgänglich ist, so zeigen Studien, dass über $80 \%$ der hospitalisierten Kinder auf der Intensivstation versterben (McCallum et al. 2000; Carter et al. 2004).

Der Sterbeort des Kindes hat großen Einfluss auf die Familie. So zeigte sich in einer Studie, dass Familien, deren Kind zu Hause verstorben ist, die Trauer besser bewältigen und weniger psychische Auffälligkeiten zeigten, als Familien, deren Kinder im Krankenhaus verstorben waren (Mulhern et al. 1983). Eine australische Studie konnte deutlich machen, dass der Sterbeort des Kindes sich auf die psychische Verfassung von Mutter und Vater des Kindes unterschiedlich auswirkt. So zeigten Väter verstärkt Depressionen, Ängste und Stress, wenn ihr Kind im Krankenhaus verstorben war. Bei den befragten Müttern ließ sich kein vergleichbares Ergebnis feststellen (Goodenough et al. 2004).

\subsection{Versorgungsstrukturen der pädiatrischen Palliativversorgung}

Viele Kinder mit LBE und LLE werden die meiste Zeit von ihren Familien zu Hause versorgt. Unterstützung bei der Pflege ihres Kindes zu Hause, im Sinne einer spezialisierten pädiatrischen Palliativversorgung (SAPV), erhalten Eltern u. a. durch niedergelassene Kinder- und Jugendärzte sowie Hausärzte, klinikgestützte, multiprofessionelle Brückenteams ${ }^{3}$, ambulante Kinderkrankenpflegedienste, ambulante Kinderhospizdienste, sozialpädiatrische Nachsorgeeinrichtungen - (diese orientieren sich am Modell des Bunten Kreises ${ }^{4}$ - und Sozialpädiatrische Zentren (Zernikow und Nauck 2008). Teilweise erfordert der Bedarf der Eltern nach kurzzeitiger physischer und psychischer Entlastung eine stationäre Unterbringung des Kindes. Es gibt in der Bundesrepublik Deutschland einige wenige regionale Kurzzeitpflegeeinrichtungen, wie z. B. die „Kleine Oase“ in

\footnotetext{
${ }^{3}$ Entwicklung aus der Kinderonkologie, stehen jedoch zunehmend auch nicht-onkologischen Patienten zur Verfügung. Interprofessionelle Versorgung des Patienten innerhalb und außerhalb der Klinik. Koordination eines Versorgungsnetzwerkes für die Familie mit Rund-um-die-Uhr-Verfügbarkeit.

41992 entwickelte die Arbeitsgemeinschaft „Förderkreis Kinderklinik Augsburg“ ein Nachsorgekonzept („Der Bunte Kreis“) für Familien mit chronisch-, krebs- und schwerstkranken Kindern. Ziel: Reibungsloser Übergang von Krankenhausbehandlung $\mathrm{zu}$ ambulanter Versorgung durch sozialmedizinische Nachsorge und Versorgungskoordination.
} 
Datteln. Daneben entstand 1998 in der Bundesrepublik Deutschland das erste Hospiz für Kinder und Jugendliche. Es gibt mittlerweile (2016) 14 solcher stationären Kinderhospize mit 143 verfügbaren Betten in Deutschland. Die meisten Kinderhospize (4) gibt es in Nordrhein-Westfalen. In sechs Bundesländern, u. a. Baden-Württemberg und Brandenburg, existieren aktuell (2016) keine Hospize für Kinder und Jugendliche (Datenerhebung Deutscher Hospiz- u. PalliativVerband). Besondere Bedeutung hat in Kinderhospizen die Arbeit und Betreuung der Eltern und Geschwister des erkrankten Kindes.

\subsubsection{Pädiatrische Palliativversorgungversorgung in Kliniken}

Nicht immer kann ein Kind im Krankheitsverlauf oder am Lebensende durchgehend zu Hause versorgt werden, weil schwierige Phasen der Erkrankung, - z. B. durch hohe Symptombelastung - eine vorübergehende stationäre Behandlung und Betreuung des Kindes notwendig machen. Es gibt verschiedene Möglichkeiten, eine palliativmedizinische Versorgung für Kinder und Jugendliche im Krankenhaus zu realisieren. Verschiedene Kliniken in Deutschland verfolgen in diesem Zusammenhang unterschiedliche Konzepte: Seit 2010 gibt es die erste Palliativstation für Kinder, Jugendliche und junge Erwachsene an der Vestischen Kinder- und Jugendklinik Datteln. Mit der Dattelner Station entstand eine multiprofessionelle und speziell auf die palliativmedizinischen Bedürfnisse von Kindern und Jugendlichen ausgerichtete Einrichtung (Kinderpalliativzentrum Datteln). Im Juni 2016 eröffnete ein Kinderpalliativzentrum am Klinikum Großhadern in München. Im Klinikum Leverkusen wurde auf der Palliativstation ein Zimmer speziell für Kinder und Jugendliche hergerichtet. Im Krankenhaus Neuwerk „Maria von den Aposteln“ in Mönchengladbach wurde eine Kinderpalliativeinheit in die allgemeinpädiatrische Station integriert. Dort ist eine sichtbare Abtrennung der beiden Bereiche (allgemeinpädiatrische Station und

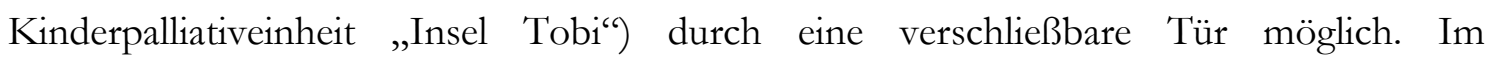
Marienhospital in Wesel entstand eine Kinderpalliativeinheit auf der Kinderintensivstation/Neonatologie.

\subsection{Das Kinderpalliativzentrum Göttingen mit Kinderpalliativzimmer}

Das Kinderpalliativzentrum Göttingen ist eine Kooperation der Klinik für Kinder- und Jugendmedizin und der Klinik für Palliativmedizin und unterstützt Kinder, Jugendliche und junge Erwachsene in der spezialisierten ambulanten und stationären Palliativversorgung. Die ambulante Palliativversorgung (SAPV) Göttingen ist Mitglied im niedersachsenweiten 
Betreuungsnetz für schwerkranke Kinder und Jugendliche Hannover. Das ärztliche Team des Kinderpalliativzentrums besteht aus Mitarbeitern des Palliativzentrums Göttingen, der Allgemein- und Neuropädiatrie, sowie der Kinderonkologie. Zusätzlich besteht eine Kooperation mit Mitarbeitern der Kinderkardiologie und pädiatrischen Intensivmedizin. Mitarbeiter aus der Gesundheits- und Kinderkrankenpflege bilden das pflegerische Team. Vervollständigt wird das Team des Kinderpalliativzentrums durch Mitarbeiter verschiedener psychosozialer Berufsgruppen. Die stationäre Versorgung von Kindern und Jugendlichen mit palliativmedizinischem Behandlungsbedarf erfolgt durch die Mitarbeiter der Klinik für Kinder- und Jugendmedizin. Eine konsiliarische Unterstützung durch Erwachsenenpalliativmediziner ist jederzeit möglich. Es finden regelmäßige Treffen des Teams des Kinderpalliativzentrums mit Besprechungen der Patientenfälle statt. Seit 2014 steht in der Klinik für Kinder- und Jugendmedizin Göttingen ein Kinderpalliativzimmer (KPZ), das in die Abteilung Neuropädiatrie integriert wurde, für die stationäre palliativmedizinische Versorgung zur Verfügung (Nauck und Gärtner 2015). Dieses soll die optimale medizinische Versorgung eines Krankenhauses mit der Privatsphäre eines Zuhauses und einer kinderpalliativmedizinischen Betreuung verbinden. In schwierigen Phasen der Erkrankung und am Lebensende soll es eine optimale medizinische, pflegerische und psychosoziale Versorgung des Kindes ermöglichen. Das KPZ befindet sich direkt zwischen den anderen Zimmern der Station und eine sichtbare Abtrennung, beispielsweise in Form einer verschließbaren Zwischentüre, existiert nicht. In Göttingen entschied man sich bewusst gegen eine Integration des KPZs in die Palliativstation für Erwachsene bzw. in die Kinderintensivstation. Auch wenn im Kinderpalliativzimmer gestorben werden darf, sollte es, wenn möglich nicht der letzte Versorgungsort des Patienten sein. In den meisten Fällen wird eine nachfolgende ambulante Versorgung zu Hause angestrebt und auch verwirklicht. Die Verortung des Zimmers auf einer Palliativoder Intensivstation hätte - nach Meinung der Verantwortlichen - diesen Weg zurück nach Hause für die Kinder und ihre Familien ein Stück weit verschlossen. Das Zimmer steht vornehmlich Patienten zur Verfügung, die eine palliativmedizinische Versorgung benötigen. Jene Patienten, die eine stationäre palliativmedizinische Betreuung erhalten, werden vom Team des Kinderpalliativzentrums mitbetreut. Das KPZ ist ein großzügig gestalteter Raum und neben dem Zimmer für das erkrankte Kind steht den Familien ein abtrennbarer Raum als Rückzugsmöglichkeit zur Verfügung. Das Zimmer verfügt über ein eigenes behindertengerechtes Bad. Die Einrichtung ist hell, freundlich und kindgerecht. Für das KPZ wurde ein Balkonzugang geschaffen, von dem aus eine Aussicht auf den Patientengarten des Palliativzentrum für Erwachsene und den Spielbereich der Klinik für 
Kinder- und Jugendmedizin möglich ist (s. Abbildung 1 und Abbildung 2). Die Ausstattung und Gestaltung des KPZs unterscheidet sich deutlich von den anderen Zimmern der Abteilung Neuropädiatrie. So ist es das einzige Zimmer mit eigenem Bad, Balkonzugang und einem Rückzugsbereich für die Familie. Das KPZ ist ein Beitrag zur Erweiterung der bestehenden kinderpalliativmedizinischen Versorgungsstrukturen in Göttingen (ambulante Betreuung im Rahmen einer spezialisierten ambulanten pädiatrischen Palliativversorgung (SAPV) durch das Kinderpalliativzentrum Göttingen, ergänzt durch den Ambulanten Kinder- und Jugendhospizdienst Göttingen bzw. die Häusliche Kinderkrankenpflege Göttingen (KIMBU) und der stationären konsiliarischen kinderpalliativmedizinischen Behandlung auf den Stationen der Klinik für Kinder- und Jugendmedizin der UMG).

Um den Erfolg des KPZs im Rahmen der täglichen Stationsarbeit zu gewährleisten und anderen Kliniken, die eine ähnliche Versorgungsstrukturerweiterung planen, eine Unterstützung zu bieten, ist es wichtig zu wissen, wie die beteiligten Versorgenden diesem gegenüberstehen. Bisher ist nicht untersucht worden, ob und inwiefern die Präsenz eines solchen Zimmers, als in direktem Zusammenhang mit Sterben und Tod stehend, wahrgenommen wird, welche Erwartungen Behandler an ein derartiges Zimmer haben und welche Schwierigkeiten sie möglicherweise in diesem Zusammenhang auf sich zukommen sehen. Es gab bislang keine Studie, die die Mitarbeiterperspektive eines entsprechenden Implementierungsprozesses untersuchte. Es ist deshalb entscheidend zu erfahren, wie die Beteiligten den Prozess erlebten und welche Vorstellungen sie von der Arbeit in und mit einem KPZ haben. Die Ergebnisse können Stärken der Integration eines solchen Zimmers auf einer Normalstation zeigen. Sie können aber auch einen Anhaltspunkt für mögliche Nachbesserungen bieten und so einen Beitrag für eine positive Wahrnehmung dieses Versorgungsmodells im Rahmen der Kinderpalliativversorgung leisten. Damit können auch Grundlagen aufgezeigt werden, die, orientiert an den Bedürfnissen der Mitarbeiter, für eine erfolgreiche Arbeit des einzelnen Mitarbeiters im KPZ bedacht werden sollten. 


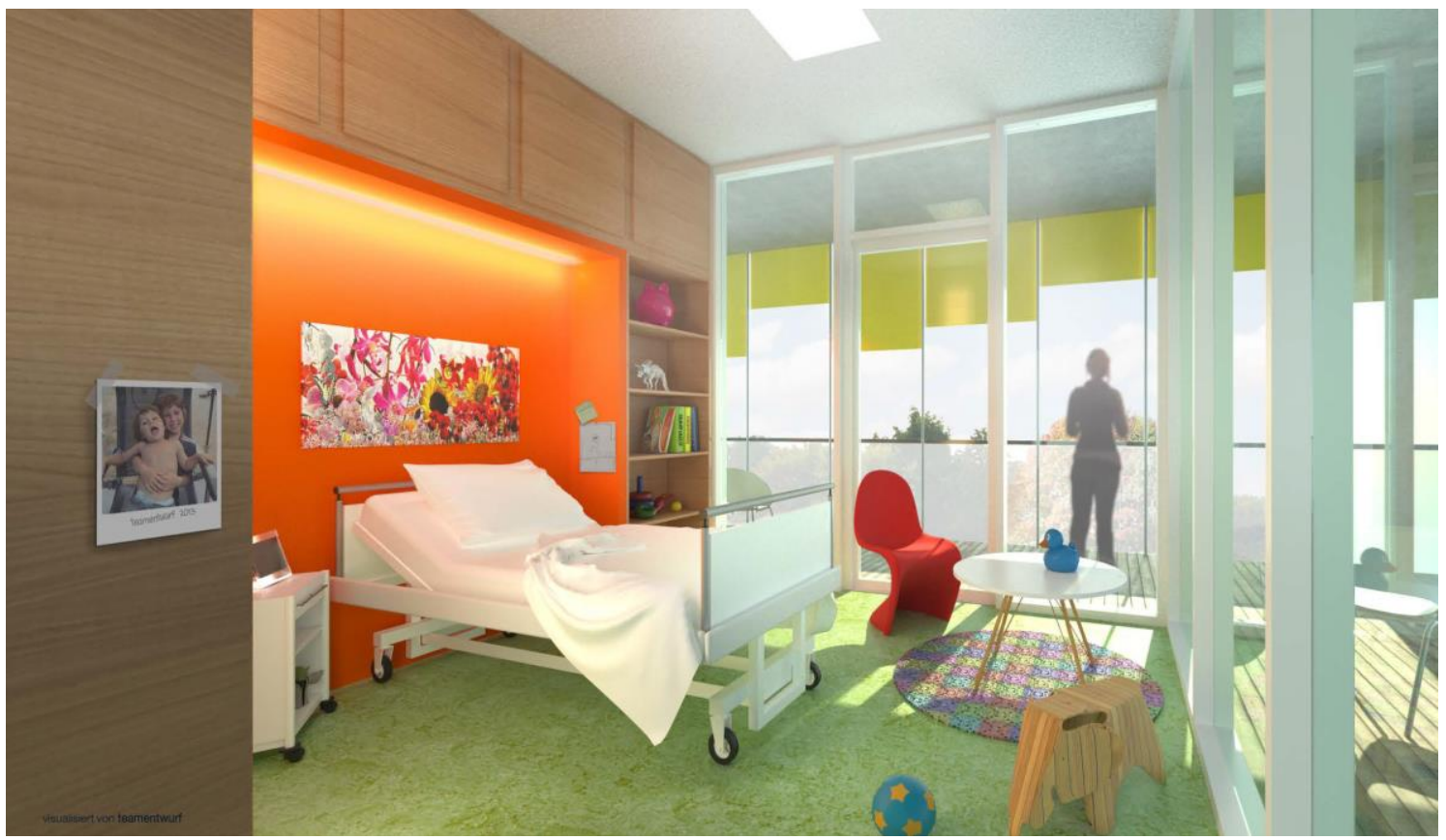

Abbildung 1 Visualisierung des KPZs (Flyer Kinderpalliativmedizin)

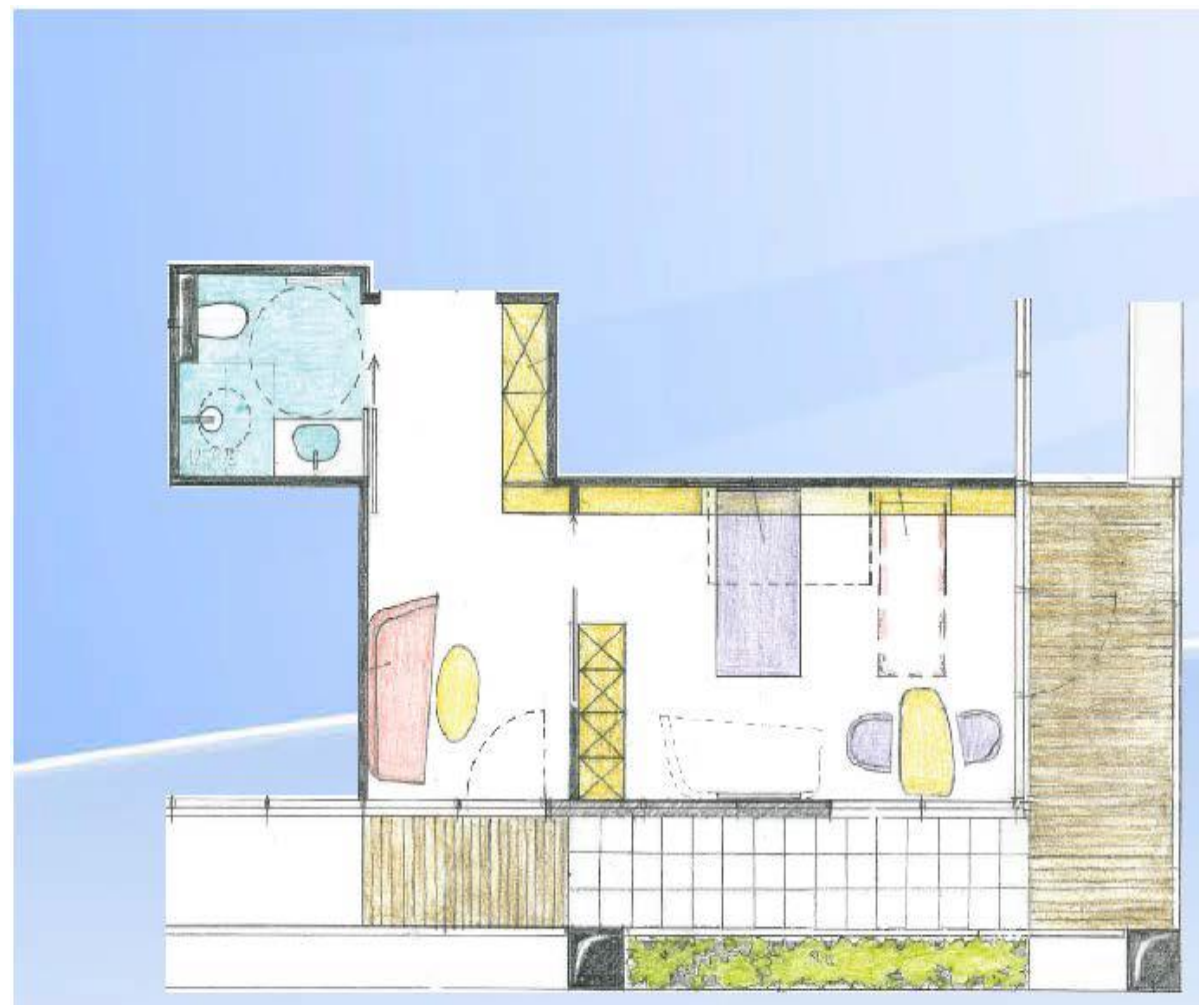

Abbildung 2 Grundriss KPZ (Flyer Kinderpalliativmedizin) 


\section{$4 \quad$ Material und Methoden}

\subsection{Wahl und Begründung der Methode}

Für die Beantwortung der Fragestellung wurde ein qualitatives Studiendesign verwendet, da mehr über die persönlichen Erfahrungen und Perspektiven der Mitarbeiter in Bezug auf den Implementierungsprozess des KPZs erfahren werden sollte. Für die Erhebung und Auswertung der Daten kam die Methode der „Grounded Theory“ nach B. Glaser und A. Strauss (2010) bzw. A. Strauss und J. Corbin (1999) zur Anwendung. Deren Ziel ist es, eine Theorie zu formulieren, die geeignet ist, das Erleben des Entstehungsprozesses des KPZs der - in die Therapie einbezogenen - Mitarbeiter zu beschreiben. Als wesentlich für die Entdeckung von Grounded Theory erweist sich eine komperative Analyse. Dieses vergleichende Vorgehen, welches schon beim theoretischen Sampling beginnt, erleichtert die „Entdeckung, Generalisierung und Spezifizierung empirisch fundierter Theorien“ (Bohnsack et al. 2003, S. 100).

Die Doktorandin erledigte alle Arbeitsschritte, beginnend mit der Terminvereinbarung, über die Interviewführung und Transkription einzelner Interviews, bis zum Kodieren selbstständig. Die Transkription von 14 der insgesamt 17 Interviews erfolgte durch eine eingewiesene studentische Hilfskraft. Es erfolgte ein regelmäßiger Austausch mit der Betreuerin des Projektes, Frau Dr. disc. pol. Gabriella Marx, und der Forschungswerkstatt der Klinik für Palliativmedizin Göttingen.

\subsection{Sampling (Zugang zum Feld)}

Für die Datenerhebung wurden Mitarbeiter der Stationen Neuropädiatrie und Pädiatrische Hämatologie und Onkologie der Klinik für Kinder und Jugendmedizin der UMG und Mitarbeiter des Kinderpalliativzentrums Göttingen, die mindestens seit Beginn der Bauphase dort beschäftigt waren und durch ihre Arbeit einen direkten Kontakt zum Kinderpalliativzimmer hatten, identifiziert und zur Teilnahme eingeladen. Dies stellte die Grundgesamtheit dar, also die Menge an Subjekten (in diesem Fall Mitarbeiter), über die die Studie eine Aussage machen sollte. Da in diesem Fall die Grundgesamtheit endlich und bekannt war, wurde eine Vollerhebung angestrebt (Brosius et al. 2008). Die Gruppe der Interviewpartner bestand aus Ärzten verschiedener Fachrichtungen (Neuropädiatrie, Onkologie, Palliativmedizin), aus Gesundheits- und Kinderkrankenpflegern und 
Berufsangehörigen verschiedener psychosozialer Berufsgruppen (Psychologen, Erzieher, Sozialpädagogen, Theologen).

Für die Rekrutierung der Interviewpartner wurden verschiedene Vorgehensweisen gewählt. Im Oktober 2014 erfolgte eine Projektvorstellung bei der Pflegedienstleitung der Station Neuropädiatrie und die Aushändigung eines Informationsschreibens (vgl. Kapitel 8, Anhang A Informationsschreiben). Die Pflegedienstleitung stellte anschließend das Projekt in einer Teambesprechung dem Team vor und hängte das Informationsschreiben am „Schwarzen Brett“ aus. Es folgte ein mehrmaliges persönliches Vorsprechen der Doktorandin auf der Station mit der Bitte um Teilnahme an der Studie. Die gleiche Vorgehensweise wurde für die Rekrutierung des Pflegepersonals der Pädiatrischen Hämatologie und Onkologie angewendet. Von einer Beteiligung des Pflegepersonals der Pädiatrischen Hämatologie und Onkologie wurde seitens der Station jedoch abgesehen, da kein direkter Kontakt mit dem Kinderpalliativzimmer im Arbeitsalltag gegeben sei. Das ärztliche Personal und die Mitglieder des Kinderpalliativzentrums wurden gezielt persönlich kontaktiert (per E-Mail, telefonisch oder Vorsprechen im Büro), nachdem die Mitgliederliste und Homepage der Klinik für Kinder- und Jugendmedizin der UMG gesichtet worden war.

Insgesamt erklärten sich 17 Mitarbeiterinnen und Mitarbeiter zur Teilnahme an der Studie bereit. Zwei von ihnen stellten sich spontan bei der persönlichen Kontaktierung zu einem Interview zur Verfügung, mit allen anderen Interviewpartnern wurden Termine vereinbart. Eine Interviewpartnerin/ein Interviewpartner meldete sich aufgrund des Aushanges, die anderen waren nach persönlicher Kontaktaufnahme zur Teilnahme an der Studie bereit. Zwei Interviewpartner mussten das Gespräch aufgrund einer spontanen Dienstplanänderung absagen, vereinbarten jedoch einen neuen Termin. Eine Interviewpartnerin/ein Interviewpartner erschien nicht zum vereinbarten Zeitpunkt und eine versuchte erneute Kontaktaufnahme scheiterte. Alle anderen Interviews fanden wie geplant statt.

Die ursprünglich angestrebte Vollerhebung wurde nicht umgesetzt, da sich nicht alle Mitarbeiter zur Teilnahme an der Studie bereit erklärten. 


\subsection{Datenerhebung}

\subsubsection{Qualitative Interviews}

Qualitative Interviews schaffen einen Rahmen, um „Situationsdeutungen oder Handlungsmotive in offener Form zu erfragen" (Flick et al. 2013, Kap. 5.2, S. 350). Es kann zwischen verschiedenen Varianten des qualitativen Interviews unterschieden werden. So kann entweder die Interviewführung durch einen vorher erstellten Fragenkatalog weitgehend festgelegt sein oder die Interviewführung ist sehr offen gehalten und es gibt kaum vorher festgelegte Fragen. Einen Mittelweg stellt das teilstandardisierte Interview dar, wofür sich auch in dieser Studie entschieden wurde. Dabei wurde im Vorfeld ein InterviewLeitfaden formuliert, der der Interviewerin eine Orientierung bot. Die folgenden Fragen dienten in den durchgeführten Interviews als Orientierungsgrundlage:

1. Wie Sie ja wissen, ist hier das neue Kinderpalliativzimmer entstanden. Erzählen Sie mir doch bitte von dem Moment, als Sie von dem Zimmer erfahren haben und was danach bis heute passiert ist in Ihrem Alltag. Bitte gehen Sie auf alle Einzelheiten ein, die Ihnen wichtig erscheinen.

2. Bitte erzählen Sie mir etwas über Ihre Tätigkeit/Aufgaben auf der Station. Erzählen Sie mir z. B. von Ihrem gestrigen Arbeitstag.

3. Wenn Sie einmal in die Zukunft blicken: Wie stellen Sie sich den Arbeitsalltag auf der Station vor, nachdem sich das Kinderpalliativzimmer auf der Station etabliert hat? Bitte gehen Sie hier auch auf Einzelheiten ein, die Ihnen wichtig sind.

Wie Rosenthal (2015) anmerkte, setzt die Erstellung eines derartigen Leitfadens voraus, dass die wissenschaftlich Tätigen über Annahmen darüber verfügen, welche Themen im entsprechenden Bereich von Relevanz sind. Der Leitfaden wurde von der Doktorandin entwickelt und anschließend im Rahmen der Forschungswerkstatt diskutiert. Der Leitfaden diente jedoch lediglich als Orientierung und die genaue Formulierung und Reihenfolge der Fragen konnte in jedem Interview individuell gewählt werden. Bei den Interviews handelte es sich um narrative Interviews, bei denen eine Erzählaufforderung im Vordergrund stand (Flick et al. 2013, Kap. 5.2) und die „Hervorlockung und Aufrechterhaltung von längeren Erzählungen“ (Rosenthal 2015, S. 162) angestrebt wurde. Diese offene Erzählaufforderung sorgte bei manchen Interviewpartnern zunächst für Verwirrung und Verunsicherung. Sie wussten zuerst nicht, wie sie auf die Art der Frage bzw. Erzählaufforderung reagieren sollten. Es wurden dann zu Beginn nur kurze Antworten gegeben. Dies hing 
möglicherweise damit zusammen, dass sie einen ausgearbeiteten Fragenkatalog mit detaillierten kurzen Fragen erwartet hatten. Im Laufe des Interviews veränderte sich dann in vielen Fällen das Antwortverhalten und längere Erzählungen konnten selbstständig generiert werden. Eine teilnehmende Person schaffte es von sich aus direkt, eine neunminütige Erzählung zu generieren und auf Nachfrage (,und wie ging es dann weiter?") nochmals zwölf Minuten frei zu erzählen.

Während des Interviews machte sich die Doktorandin Notizen, um immanente, ebenfalls erzählgenerierende Nachfragen stellen zu können, wenn die freie Erzählung des Interviewpartners bzw. der Interviewpartnerin stoppte. Dabei musste darauf geachtet werden, keine geschlossenen Fragen zu stellen. Bei sogenannten geschlossenen Fragen werden die Antwortmöglichkeiten durch die Frage selbst vorgegeben. Die Interviewpartnerin/der Interviewpartner kann der Frage entweder inhaltlich zustimmen oder nicht und die Frage nur mit Ja oder Nein beantworten. Es ist der Interviewpartnerin/dem Interviewpartner dabei nicht möglich, eigene Schwerpunkte in seiner Beantwortung zu setzen. Demgegenüber stehen offene Fragen, die verschiedene Antwortmöglichkeiten zulassen und die Gesprächsinitiative an die Befragte oder den Befragten abgeben. Sie werden häufig mit einem W-Pronomen eingeleitet (z. B. Wie, Warum, Welche). So lassen sich am besten z. B. Wünsche oder auch Meinungen des Interviewpartners erfahren (Müller-Dofel 2013). Es kann zusätzlich erzählgenerierend sein, die Nachfragen in der Sprache der Interviewten zu verfassen und von ihm verwendete Begriffe und Ausdrücke aufzugreifen (Rosenthal 2015, S. 174). Nach Rosenthal (2015) können sechs Typen des narrativen Nachfragens unterschieden werden. Diese kamen auch während der Interviews zum Einsatz. Zwischen folgenden Fragetypen konnte situationsabhängig gewählt werden:

1. Ansteuern einer Lebensphase: z. B. können sie mir von Ihrer Zeit in der ambulanten Kinderkrankenpflege erzählen?

2. Eröffnung eines temporalen Rahmens: z. B. können Sie mir von der Eröffnung des Kinderpalliativzimmers erzählen und wie Sie seitdem den Stationsalltag erlebt haben?

3. Das Ansteuern einer benannten Situation: z. B. Sie erwähnten vorhin die Situation (X), können Sie mir diese Situation einmal genau erzählen?

4. Ansteuern einer Erzählung zu einem erwähnten Argument: z. B. können Sie sich an eine Situation erinnern, als Sie sich von Ihrem Vorgesetzten allein gelassen fühlten? 
5. Ansteuern von Tradiertem bzw. Fremderlebten: z. B. können Sie sich noch an eine Situation erinnern, als Ihnen davon erzählt wurde, was Aufklärungsgespräche mit Eltern ergaben?

6. Ansteuern von Zukunftsvorstellungen oder von Phantasien: z. B. können sie mir erzählen, welche Hoffnungen Sie an das Kinderpalliativzimmer haben?

Den Studienteilnehmern wurde freigestellt, wann und wo das Interview stattfinden sollte. Die Interviews fanden hauptsächlich in Büros oder Aufenthaltsräumen der Klinik für Kinder- und Jugendmedizin, also am Arbeitsplatz der Interviewpartner, statt. Drei Interviews wurden in den Räumlichkeiten der Mildred-Scheel-Akademie Göttingen durchgeführt. Alle Mitarbeiter entschieden sich für einen Termin, der im direkten Zusammenhang mit ihrer Arbeitszeit stand, also direkt vor, direkt nach oder während ihrer Arbeitszeit. Von der Doktorandin wurde ursprünglich eine Interviewdauer von ca. 60 Minuten veranschlagt, welche den Interviewpartnern auch bei der Terminvereinbarung genannt wurde. Nach den ersten fünf Interviews stellte sich jedoch heraus, dass die meisten Interviews während der Arbeitszeit oder kurz vor Dienstbeginn der Mitarbeiter stattfanden und viele von ihnen deshalb weniger Zeit erübrigen konnten. Die angestrebte Interviewdauer wurde deshalb auf 45 Minuten reduziert. Die Interviews waren letztlich zwischen 38 und 92 Minuten lang. Auf diesen Aspekt - Interviewdauer und Interviewzeitpunkt - wird im Kapitel 6.9.1 Interviewsituation noch näher eingegangen.

\subsubsection{Kurzfragebogen}

Im Anschluss an das jeweilige Interview wurden die Teilnehmer gebeten, einen Kurzfragebogen auszufüllen (vgl. ). Dieser beinhaltete in erster Linie Fragen nach personalen und demographischen Eigenschaften der Interviewpartner. Dazu gehörten Alter, Geschlecht, Familienstand, Frage nach eigenen Kindern, Ausbildung, Beruf und Konfession. Der Fokus lag vor allem auf der Erhebung von Eigenschaften der Befragten, um Verbindungen zwischen den demographischen Eigenschaften der Interviewpartner und ihren Meinungen, Auffassungen und Handlungsweisen herzustellen (Schnell 2013, Kap. 7). Zusätzlich wurden Verhaltensfragen gestellt, z. B. die Frage nach der aktiven Ausübung einer Religion. Dieser Aspekt erschien bedeutungsvoll, da ein Zusammenhang zwischen aktiver Ausübung einer Religion und der Verarbeitung von Tod und Sterben möglich schien. Bei den Fragen-Strukturtypen des Kurzfragebogens wurden geschlossene Fragen gewählt und bei den Antwortmöglichkeiten zwischen Alternativvorgaben (ja/nein), ungeordneter Mehrfachvorgabe (mehrere Antwortmöglichkeiten können ausgewählt 
werden) und Hybridfragen variiert. Bei Letzteren besteht die Möglichkeit, zusätzlich eine freie Antwort zu geben, falls keine der Vorgaben der/dem Interviewten als Antwort passend erscheint. Es wäre ebenfalls möglich gewesen, diese Daten im Rahmen des Interviews zu erfragen. Es bestand jedoch die Befürchtung, damit in ein ,Frage-AntwortSpiel' abzugleiten und den narrativen Charakter des Interviews zu verlieren. Die Ergebnisse des Fragebogens wurden keiner statistischen Analyse unterzogen, sondern dienten der Anreicherung der qualitativ erhobenen Daten sowie der Beschreibung der Stichprobe (vgl. Kapitel 5.1 Stichprobe (Sample)).

\subsubsection{Transkription}

Die Interviews wurden audiodigital aufgezeichnet, worüber die Interviewpartner vor Beginn des Interviews aufgeklärt wurden (vgl. Kapitel 4.5 Datenschutz und Genehmigung durch die Ethikkommission). Die Interviews wurden anschließend wörtlich transkribiert. „Unter Transkription versteht man die graphische Darstellung ausgewählter Verhaltensaspekte von Personen, die an einem Gespräch (z. B. einem Interview oder einer Alltagsunterhaltung) teilnehmen“ (Flick et al. 2013, S. 438). In dieser Studie wurde die Form der literarischen Umschrift angewendet, wo Abweichungen von Standardsprache, z. B. Elision (,wolln“ für „wollen“) oder Assimilation (,willste“ für „willst du“) berücksichtigt werden. Neben den verbalen Merkmalen (gesprochenes Wort) wurde auch deren lautliche Umsetzung (prosodische Merkmale), wie Lautstärkevariation, und nonverbale Äußerungen, wie Lachen und Husten, festgehalten (Flick et al. 2013, S. 438), vgl. dazu auch Anhang C Transkriptionsregeln. Durch dieses Vorgehen sollten Einzigartigkeiten des Redeverhaltens eindeutig abgebildet werden (Rickheit et al. 2003, S. 96). Diese Art der Dokumentation ist für interpretative Analyseverfahren notwendig, da Wortabbrüche, Wiederholungen, Betonungen, Lautstärke usw. Aufschluss über das Erleben und die Perspektive der interviewten Person geben können und daher systematisch in die Analyse einbezogen wurden. Die Transkription erfolgte mithilfe der Transkriptionssoftware „f4transkript“. Wie zu Beginn des Kapitels angeführt, wurden 14 Interviews von einer eingewiesenen studentischen Hilfskraft transkribiert. Die Doktorandin transkribierte drei Interviews selbstständig und überprüfte die von der studentischen Hilfskraft transkribierten Interviews, um sicherzustellen, dass einheitliche Maßstäbe bei der Transkription verwendet wurden. 


\subsection{Datenauswertung}

Für die Erhebung und Auswertung der Daten wurde die Methode der Grounded Theory verwendet. Die Methode wurde 1967 von Strauss und Glaser entwickelt und bildet ein Rahmenkonzept für die Datensammlung, Datenanalyse und datenbasierte, d.h. aus den Daten heraus entwickelte Theorieformulierung.

Strauss und Corbin haben die Methode weiterentwickelt und definieren die Grounded Theory als „eine gegenstandsverankerte Theorie, die induktiv aus der Untersuchung des Phänomens abgeleitet wird, welches sie abbildet"(Strauss und Corbin 1999, S. 7). Glaser und Strauss fordern für die Theoriebildung, dass Datenerhebung, Kodierung und Datenanalyse keinem linearen Prozess folgen, sondern vielmehr gleichzeitig ablaufen sollen (Glaser und Strauss 2010). Strauss und Corbin behalten dieses Vorgehen bei und begründen es damit, dass die Trennung von Datenerhebung und -auswertung aufgehoben ist und stattdessen die Auswertung die Datenerhebung leitet (Strauss und Corbin 1999). Die Datensammlung orientiert sich am Theoretischen Sampling, worauf in Abschnitt 4.4.4 noch näher eingegangen wird. Parallel dazu fand bereits eine erste Analyse der Daten statt und erste Konzepte konnten entwickelt werde. Während des kompletten Forschungsprozesses wurden Memos verfasst, d.h. schriftliche Notizen, die parallel zum Interviewverlauf oder zur Datenanalyse angefertigt werden und verschiedene Formen annehmen konnten. Im Rahmen dieses Vorhabens wurden Memos jeweils direkt nach einem Interview verfasst und die Eindrücke der Doktorandin bezüglich der Interviewsituation festgehalten.

\subsubsection{Offenes Kodieren}

Die Datenanalyse (Kodieren) gliederte sich in drei Abschnitte, nämlich das offene, das axiale und das selektive Kodieren, auf die im Folgenden näher eingegangen wird:

Das offene Kodieren stellt den Beginn der Analysearbeit dar und dient der Konzeptualisierung der Daten. Konzepte sind laut Strauss und Corbin „Etiketten, die einzelnen Ereignissen, Vorkommnissen oder anderen Beispielen für Phänomene zugeordnet werden“ (Strauss und Corbin 1999, S. 43). Um die Konzepte zu spezifizieren, mussten Vergleiche angestellt und Fragen an den Test gestellt werden. Dabei ist es wichtig, nicht $\mathrm{zu}$ deskriptiv an den Text heranzugehen, sondern darauf $\mathrm{zu}$ achten, welche Sichtweisen zwischen den Zeilen, also hinter den gesprochenen und offensichtlichen Inhalten sichtbar werden. Auf diese Weise ist ein Zugang zu den die Handlung bestimmenden Haltungen möglich. So erhielten z. B. die Aussagen von Interviewpartnern: 
„auf der Intensivstation is ja so alle kämpfen da um das Kind und keiner kann loslassen , ja aber (im ersten) Schritt da sieht man eigentlich man kann dem eigentlich nich mehr belfen und trotzdem versucht man und versucht und versucht" (KPZ 12, Z. 500-502)

und

„weil in der Klinik is es ja alle bemühen sich alle Menschen bemühen sich dass das Kind geheilt werden kann" (KPZ 6, Z. 342-344)

das Konzept „schwere Akzeptanz sterbender Kinder im kurativen System“. Dieses Konzept soll nun etwas genauer erläutert werden: Aufgrund des medizinischen Fortschrittes versterben heute immer weniger Kinder in Deutschland und der Tod eines Kindes wird deshalb ein Stück weit zum gesellschaftlichen Tabuthema, weil er eine Umkehr der gefühlten natürlichen Ordnung darstellt. Gerade weil sich die Medizin in den letzten Jahren stark weiterentwickelt hat und immer mehr Krankheiten erfolgreich behandelt werden können, besteht die Hoffnung und vielleicht auch ein gewisser Anspruch in der Gesellschaft, dass jedes Kind geheilt werden kann. Der Tod eines Kindes, trotz aller bestehenden medizinischen Möglichkeiten, ist deshalb eine fachliche und emotionale Herausforderung für die beteiligten Mitarbeiter. Diese - fast schon verzweifelt wirkenden - Bemühungen und das Nicht-Aufgeben-Können kommen in den gewählten Zitaten gut zum Ausdruck („versucht man und versucht und versucht“, „alle bemühen sich“). Es scheint schwer zu akzeptieren zu sein, wenn alle Bemühungen scheitern (,alle kämpfen [...] und keiner kann loslassen").

Der Kodierprozess erfolgte als Zeile-für-Zeile-Analyse, bei der die Interviews Zeile für Zeile, Phrase für Phrase und manchmal auch Wort für Wort analysiert wurden, weil diese Methode nach Strauss und Corbin (1999, S. 53) „die detaillierteste Art der Analyse, aber auch die ergebnisreichste“ ist.

Das Kodieren erfolgte mithilfe der Datenanalyse Software MAXQDA, womit sich die Daten organisieren, kodieren und kategorisieren ließen.

\subsubsection{Axiales Kodieren}

Anschließend erfolgt der Prozess des Kategorisierens. Im Laufe des Kodierens fiel durch das Vergleichen der Konzepte auf, dass sich einzelne von ihnen auf ein ähnliches Phänomen bezogen. Diese Konzepte wurden dann unter einem Konzept höherer Ordnung, der abstrakteren Kategorie, zusammengefasst (Strauss und Corbin 1999). 
So wurden die Konzepte „schwere Akzeptanz, sterbender Kinder im kurativen System" und "psychische Belastung durch Sterbebegleitung" der Kategorie „Sterben als professionelle Herausforderung" zugeordnet.

Die sich bildenden Kategorien werden während des axialen Kodieren immer weiter verfeinert, differenziert und es werden Verbindungen zwischen ihnen hergestellt, um die vorhandene Datenmenge zu verdichten. Zur Entwicklung der einzelnen Kategorien kann man sich ein paradigmatisches Modell zur Hilfe nehmen. Dieses Modell geht auf Strauss zurück und wurde von Strauss und Corbin weiterentwickelt. In dieser Studie wurde das Modell von Strauss und Corbin verwendet, welches Jörg Strübing noch weiter ausdifferenzierte. Dieses Kodierparadigma befragt ein Phänomen nach den Phänomenen verursachenden Bedingungen, dem Kontext, den sich aus dem Phänomen ergebenden Handlungsstrategien und deren intervenierenden Bedingungen sowie den Konsequenzen. Dieses Modell ermöglicht es, die Daten systematisch zu überdenken und komplexe Beziehungsgeflechte herauszuarbeiten. Bei der Entwicklung der Kategorien wurden zudem die jeweiligen Eigenschaften und Dimensionen herausgearbeitet, was dazu dient, Verbindungen zwischen Haupt- und Subkategorien zu identifizieren (Strauss und Corbin 1999). Im Zentrum des Kodierparadigmas steht das Phänomen, welches die Kategorie beschreibt. Dieses kann durch Fragen an den Text identifiziert werden, die beispielsweise lauteten: Worum dreht sich die Handlung? Die ursächliche Bedingung bezeichnet jene Ereignisse, die zur Existenz des Phänomens beitragen. Es ist möglich, dass nicht nur eine Bedingung ursächlich für das Auftreten eines Phänomens ist, sondern mehrere. Der Kontext stellt die Ausprägungen für die aktuelle Fragestellung dar. Die Handlungsstrategien dienen der Bewältigung des Phänomens unter den gegebenen Bedingungen. Sie sind dabei zweckgerichtet und zielorientiert. Zusätzlich werden die auf das Phänomen folgenden Konsequenzen dargelegt, die aus den Handlungsstrategien resultieren (Strauss und Corbin 1999; Strübing 2008).

Beim axialen und offenen Kodieren handelte es sich zwar um getrennte Analyseschritte, im Forschungsverfahren findet allerdings ein ständiges Hin- und Herwechseln zwischen den beiden Schritten statt.

Die Bezeichnung der Konzepte und der Kategorien stammten entweder aus den bereits vergebenen Konzeptnamen, der Fachliteratur oder dem Text selbst. Letztere bezeichnet man als „In-vivo-Kodes“, weil sie dem Sprachgebrauch der Interviewpartner entstammen. So wurde z. B. der „In-vivo-Kode“ „ob ich echt ein Todesengel bin" verwendet, um zu 
beschreiben, mit welchen Selbstzweifeln die Angestellten zu kämpfen hatten, wenn wiederholt von ihnen versorgte Patienten verstarben.

\subsubsection{Selektives Kodieren}

Im Auswertungsprozess entstanden elf verschiedene Kategorien. Im letzten Analyseschritt, dem selektiven Kodieren, ergab sich die Kernkategorie (das zentrale Phänomen) aus den Daten, die mit den anderen Kategorien In-Beziehung gesetzt werden konnte, weil sich diese um die Kernkategorie anordneten. Für die Identifikation der Kernkategorie muss der „rote Faden“ in einer zu erzählenden Geschichte herausgearbeitet werden, d.h. das zentrale Phänomen musste gesucht und benannt werden (Strauss und Corbin 1999). In dieser Studie konnte die Kategorie „Durch KPZ entsteht „Klassengesellschaft" auf der Station“ als Kernkategorie bestimmt werden. Es ließen sich Verbindungen von dieser zu den anderen zehn Kategorien herstellen. Eine grafische Darstellung der so entstandenen, in den Daten gegründeten und entlang des Kodierparadigmas entwickelten Theorie mit der Kernkategorie im Zentrum findet sich in Kapitel 5.2 Präsentation der Kategorien.

\subsubsection{Theoretisches Sampling}

Glaser und Strauss fordern für den Datenerhebungsprozess die Anwendung eines theoretischen Samplings. Dieses Vorgehen soll die Generierung der Theorie unterstützen, indem Daten parallel erhoben, kodiert und analysiert werden. Währenddessen wird darüber entschieden, wo und welche Daten als nächstes erhoben werden (Glaser und Strauss 2010, S. 61). Theoretisches Sampling meint dabei die Auswahl „auf der Basis von Konzepten, die eine bestätigte theoretische Relevanz für die sich entwickelnde Theorie besitzen“ (Strauss und Corbin 1999, S. 149). Bestätigte theoretische Relevanz meint, dass Konzepte als wichtig erscheinen, weil sie entweder wiederholt vorkommen oder gänzlich abwesend sind. Die Samplingstrategie unterscheidet sich in den drei Phasen des Kodierverfahrens: So ist das Sampling während des offenen Kodierens ebenfalls sehr offen, weil möglichst viele relevante Kategorien identifiziert werden sollen. Dabei werden die Daten gezielt durchgegangen und von einem Interview zum nächsten gewechselt. Wie in Abschnitt 4.4.2 erklärt, dient das axiale Kodieren dem In-Beziehung-Setzen der Kategorien zueinander. Das Sampling während dieser Auswertungsphase dient der Aufdeckung und Bestätigung der Verbindungen. Beim selektiven Kodieren muss zu einem diskriminierenden Sampling übergegangen werden, bei dem gezielt Daten ausgewählt werden, die die Beziehungen um die Hauptkategorien verdichten und die Ausarbeitung bisher wenig entwickelter Kategorien ermöglichte. Dieser Prozess soll solange erfolgen, bis eine theoretische Sättigung der 
Kategorien erreicht wird. Theoretische Sättigung ist laut Glaser und Strauss dann erreicht, wenn der Forscher keine zusätzlichen Daten mehr finden, mit denen zusätzliche Eigenschaften der Kategorie entwickelt werden können (Glaser und Strauss 2010, S. 77). In der vorliegenden Studie wurde, wie in Kapitel 4.2 Sampling (Zugang zum Feld) bereits erwähnt, eine Vollerhebung angestrebt. Ziel war es, alle Interviews in den ersten sechs Monaten nach Eröffnung des KPZs zu führen, um seinen Einfluss auf das Erleben der Mitarbeiterinnen und Mitarbeiter während der Anlaufphase des Zimmers zu erfassen. Eine „Gewöhnung“ des Teams an das Zimmer bzw. ein unterschiedlicher „Gewöhnungszustand“ der Mitarbeiterinnen und Mitarbeiter sollte weitestgehend vermieden werden. Wenn zeitliche Gründe eine gleichzeitige Erhebung, Kodierung und Analyse verhindern, so schlagen Glaser und Strauss vor, das Theoretische Sampling mit den zuvor erhobenen Daten (transkribierte Interviews) durchzuführen (Glaser und Strauss 2010, S. 86). Hätten sich alle potentiellen Interviewpartner zu einem Gespräch bereit erklärt, wäre dieses Vorgehen gewählt worden. Da aber nur 17 Mitarbeiter in ein Interview einwilligten, wurden alle geführten Interviews ausgewertet und ein Theoretisches Sampling konnte nicht durchgeführt werden. Auf diesen Aspekt wird in Kapitel 6.9.2 Fehlendes Theoretisches Sampling noch näher eingegangen. Die 17 geführten Interviews erlaubten eine Theoretische Sättigung der Kategorien im Sinne von Glaser und Strauss.

\subsection{Datenschutz und Genehmigung durch die Ethikkommission}

Zu Beginn der Studie wurde der Ethik-Kommission der UMG unter der Antragsnummer 15/10/14 das Studienprotokoll zur Begutachtung vorgelegt. Die Ethik-Kommission entschied in ihrer Sitzung vom 23.10.2014, dass keine ethischen oder rechtlichen Bedenken gegen das vorgelegte Studienvorhaben vorlägen.

Alle Studienteilnehmer wurden ausführlich mündlich und schriftlich über die Studie aufgeklärt (vgl. Kapitel 8 Anhang A Informationsschreiben und Anhang B Intervieweinwilligung) und stimmten in der Einverständniserklärung der Archivierung der Audioaufzeichnungen währende der Studiendauer zu. Nach Abschluss der Studie werden die Prüfungsunterlagen (hier: pseudonymisierte Transkripte) gemäß den Bestimmungen des Datenschutzes verwaltet und für die Dauer von 10 Jahren nach Studienende aufbewahrt. Die Daten werden nicht an Dritte weitergegeben.

Die Teilnehmer konnten jederzeit und ohne Angabe von Gründen ihr Einverständnis zurückziehen und aus der Studie ausscheiden, ohne dass sich daraus Nachteile für sie ergeben hätten. Bei Widerruf der Zustimmung oder auf besonderen Wunsch wären die 
Aufzeichnungen umgehend und endgültig gelöscht worden. Nach Abschluss des Projektes, spätestens aber nach Erscheinen der Publikationen, werden die Audioaufzeichnungen unwiederbringlich zerstört.

Um die Anonymität der Interviewpartner zu wahren, wurden die Interviews nicht in ihrer Gesamtheit in dieser Arbeit abgedruckt, sondern nur einzelne Ausschnitte und Zitate verwendet. Da es sich bei der gezogenen Stichprobe um einen kleinen Personenkreis handelt, der sich untereinander aufgrund der engen Zusammenarbeit persönlich kennt, werden in den veröffentlichten Zitaten im Auswertungsteil Auslassungen vorgenommen, wenn gemachte Äußerungen Rückschlüsse auf eine einzelne Person ermöglichen könnten. Individuelle sprachliche Merkmale wie die Verwendung spezieller Redewendungen und dialektale Besonderheiten wurden geglättet. Es wurden jedoch keine inhaltlichen Veränderungen der Aussagen vorgenommen. 


\section{$5 \quad$ Ergebnisse}

Im Folgenden werden die aus den Interviews hervorgegangenen Ergebnisse dargestellt und erläutert. Um diesen Generierungsprozess nachvollziehbar zu gestalten und die Interpretationen zu belegen, wurden aussagekräftige Zitate aus den Interviews in den Fließtext eingebunden. Sie wurden in Anführungszeichen („), kursiv und eingerückt dargestellt. Wie in Kapitel 4.5 Datenschutz und Genehmigung durch die Ethikkommission erläutert, fanden für eine bessere Lesbarkeit der Zitate sprachliche Glättungen, aber keine inhaltlichen Änderungen der Aussagen statt. Füllwörter und nonverbale Äußerungen wurden - falls sie für die Analyse nicht erforderlich waren - zur Verbesserung der Lesbarkeit entfernt. Die literarische Umschrift (vgl. Kapitel 4.3.3 Transkription) wurde jedoch beibehalten. Die Zeilenangaben der Zitate stammen aus den Originaltranskripten. Sprachliche Glättungen und Auslassungen können dazu führen, dass diese Zeilenangaben nicht mit der aktuellen Zeilenzahl übereinstimmen.

Bei der Befragung der 17 Mitarbeiter und qualitativen Interviewauswertung zeigte sich ein unterschiedliches Erleben des Entstehungsprozesses und verschiedene Erwartungen an das KPZ. In der Ergebnisdarstellung wurden Zahlwörter - z. B. häufig, mehrfach, wenige verwendet, um zu verdeutlichen, ob es sich dabei um eine individuelle Wahrnehmung handelte oder ob dies von verschiedenen Mitarbeitern auf diese Art wahrgenommen worden war. Dies diente lediglich der Veranschaulichung und ist nicht mit einer quantitativen Auswertung gleichzusetzen. Bei dem gewählten methodischen Vorgehen ist die Quantität nicht entscheidend, sondern die vorhandene Varianz soll gleichberechtigt abgebildet werden, um auf einem abstrakten Niveau möglichst dichte Zusammenhänge darstellen zu können. Des Weiteren sei angemerkt, dass es sich nicht um eine Darstellung von „Wahrheit“, sondern um die subjektiven Empfindungen und Wahrnehmungen der Interviewpartner handelt. Dies ist vor allem dann von Bedeutung, wenn Interviewpartner Aussagen zu Kollegen oder Eltern und Patienten machen.

\subsection{Stichprobe (Sample)}

Für die Studie wurden 17 Mitarbeiter der Abteilungen Neuropädiatrie und Pädiatrische Hämatologie und Onkologie der Klinik für Kinder- und Jugendmedizin der UMG, sowie Mitarbeiter des Kinderpalliativzentrums Göttingen befragt. Zu den Abteilungsmitarbeitern zählen neben Ärzten und Gesundheits- und Kinderkrankenpflegern auch Mitarbeiter aus 
dem psychosozialen Bereich (z. B. Psychologen, Erzieher, Sozialpädagogen). Das Kinderpalliativzentrum Göttingen setzt sich aus Ärzten unterschiedlicher Fachrichtungen, Palliativfachkräften aus der Gesundheits- und Kinderkrankenpflege und Mitarbeitern verschiedener psychosozialer Berufsgruppen der Kinderklinik Göttingen und der Elternhilfe für das Krebskranke Kind Göttingen e.V. zusammen (Kinderpalliativzentrum Göttingen). Eine genaue Darstellung des Samples ist der folgenden Tabelle $1 \mathrm{zu}$ entnehmen:

\begin{tabular}{|c|c|c|}
\hline Eigenschaften: & Anzahl: & $\begin{array}{l}\text { Prozentualer Anteil } \\
\text { (gerundet): }\end{array}$ \\
\hline \multicolumn{3}{|l|}{ Geschlecht: } \\
\hline $\begin{array}{l}\text { Weiblich } \\
\text { Männlich }\end{array}$ & $\begin{array}{c}12 \\
5\end{array}$ & $\begin{array}{l}70,6 \\
29,4\end{array}$ \\
\hline \multicolumn{3}{|l|}{ Alter: } \\
\hline $\begin{array}{l}20-29 \\
30-39 \\
40-49 \\
50-59\end{array}$ & $\begin{array}{l}2 \\
3 \\
3 \\
9 \\
\end{array}$ & $\begin{array}{l}11,8 \\
17,6 \\
17,6 \\
52,9\end{array}$ \\
\hline \multicolumn{3}{|l|}{ Familienstand: } \\
\hline $\begin{array}{l}\text { In Partnerschaft lebend } \\
\text { Ledig } \\
\text { Keine Angabe }\end{array}$ & $\begin{array}{c}13 \\
3 \\
1 \\
\end{array}$ & $\begin{array}{c}76,4 \\
17,6 \\
5,9 \\
\end{array}$ \\
\hline \multicolumn{3}{|l|}{ Kinder: } \\
\hline $\begin{array}{l}\mathrm{Ja} \\
\text { Nein }\end{array}$ & $\begin{array}{l}9 \\
8 \\
\end{array}$ & $\begin{array}{l}52,9 \\
47,1 \\
\end{array}$ \\
\hline \multicolumn{3}{|l|}{ Schulabschluss: } \\
\hline $\begin{array}{l}\text { Realschulabschluss } \\
\text { Fachhochschulreife } \\
\text { Allgemeine Hochschulreife }\end{array}$ & $\begin{array}{c}3 \\
3 \\
11 \\
\end{array}$ & $\begin{array}{l}17,6 \\
17,6 \\
64,7 \\
\end{array}$ \\
\hline \multicolumn{3}{|l|}{ Ausbildungsabschluss: } \\
\hline $\begin{array}{l}\text { Beruflich-betrieblich (Lehre) } \\
\text { Beruflich-schulisch } \\
\text { (Berufsfachschule) } \\
\text { Hochschule }\end{array}$ & $\begin{array}{l}9 \\
1 \\
7\end{array}$ & $\begin{array}{c}52,9 \\
5,9 \\
41,2\end{array}$ \\
\hline \multicolumn{3}{|l|}{ Ausgeübter Beruf: } \\
\hline $\begin{array}{l}\text { Ärztin/Arzt } \\
\text { Pflegerin/Pfleger } \\
\text { Andere }\end{array}$ & $\begin{array}{l}4 \\
9 \\
4 \\
\end{array}$ & $\begin{array}{l}23,5 \\
52,9 \\
23,5 \\
\end{array}$ \\
\hline \multicolumn{3}{|l|}{ Erfahrung im derzeitigen Beruf: } \\
\hline $\begin{array}{l}\text { 1-15 Jahre } \\
\text { 16-30 Jahre } \\
\text { 31-45 Jahre } \\
\end{array}$ & $\begin{array}{l}5 \\
9 \\
3 \\
\end{array}$ & $\begin{array}{l}29,4 \\
52,9 \\
17,6 \\
\end{array}$ \\
\hline \multicolumn{3}{|l|}{ Gläubig/spirituell: } \\
\hline $\begin{array}{l}\text { Ja } \\
\text { Nein } \\
\text { Weiß nicht }\end{array}$ & $\begin{array}{c}13 \\
1 \\
3 \\
\end{array}$ & $\begin{array}{c}76,4 \\
5,9 \\
17,6 \\
\end{array}$ \\
\hline \multicolumn{3}{|l|}{ Aktiv eine Religion ausübend: } \\
\hline $\begin{array}{l}\text { Ja } \\
\text { Nein }\end{array}$ & $\begin{array}{c}10 \\
7\end{array}$ & $\begin{array}{l}58,8 \\
41,2\end{array}$ \\
\hline
\end{tabular}

Tabelle 1 Eigenschaften Sample 


\subsection{Präsentation der Kategorien}

Die Ergebnisse (vgl. Abbildung 3 Kodierparadigma) der hier vorgestellten Untersuchung werden entlang des Kodierparadigmas, in Anlehnung an Strübing (2008), dargestellt. Während des Auswertungsprozesses konnte das Phänomen „Durch KPZ entsteht „Klassengesellschaft" auf der Station" im Zentrum als zentrale Kategorie herausgearbeitet werden. Darum gruppieren sich die Kontextkategorien, ursächliche Bedingungen, eine Handlungsstrategie mit intervenierenden Bedingungen und Phänomenkonsequenzen (zum Verständnis des Kodierparadigmas vgl. Kapitel 4.4.2 Axiales Kodieren). Das paradigmatische Modell kommt sowohl bei der Erklärung der zentralen Kategorie und ihren Verbindungen mit den anderen herausgearbeiteten Kategorien als auch bei der detaillierten Erläuterung jeder einzelnen Kategorie zum Einsatz.

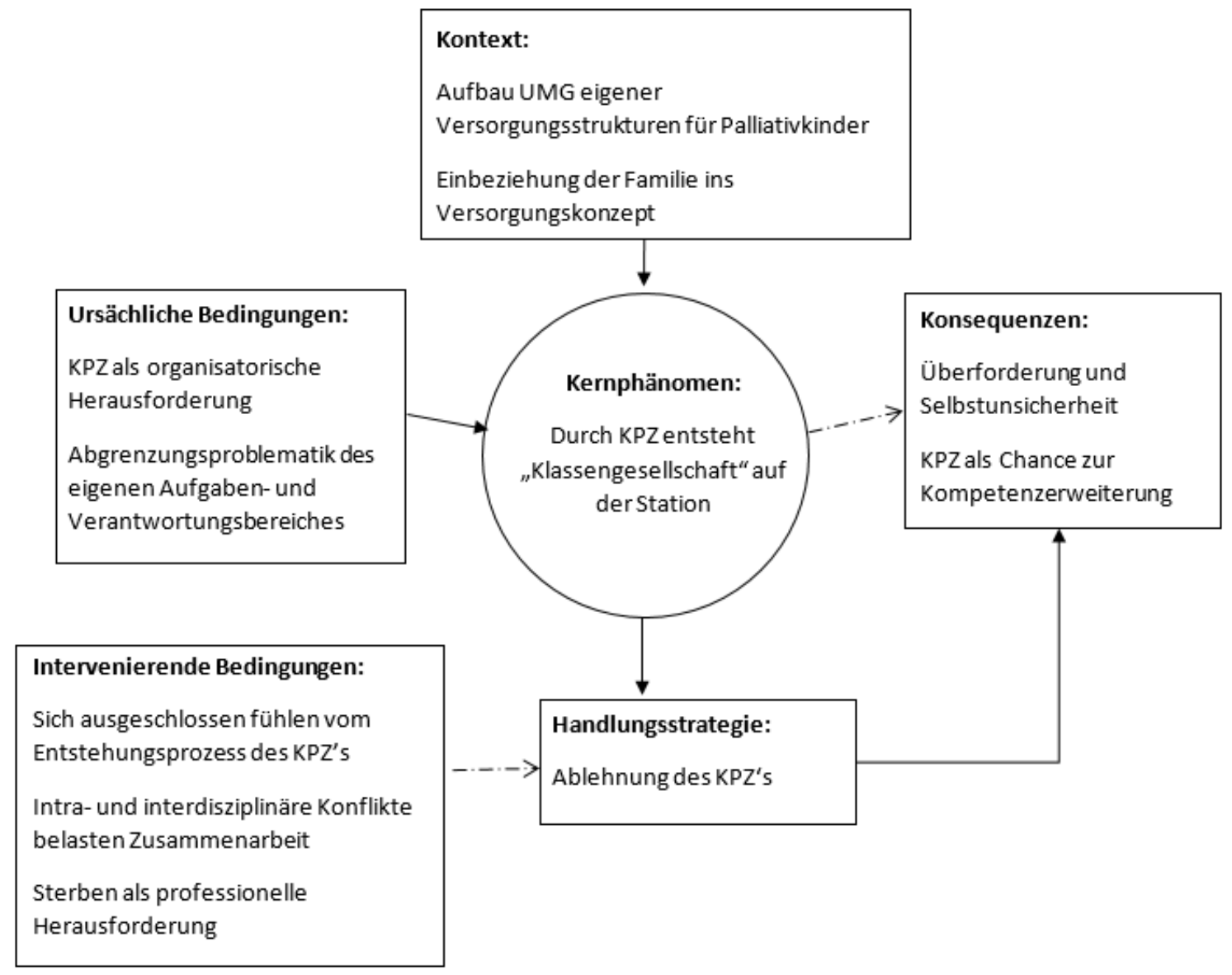

Abbildung 3 Kodierparadigma

Bevor im Folgenden eine genaue Erläuterung der einzelnen Kategorien und ihrer Verbindungen untereinander erfolgt, soll zunächst eine kurze Zusammenfassung gegeben werden, die das Verständnis der Abbildung 3 erleichtert und einen Einstieg in die 
Ergebnispräsentation darstellt: Während der Datenanalyse konnte herausgearbeitet werden, dass durch das KPZ das Gefühl einer entstehenden „Klassengesellschaft“ auf der Station bestand. Dies war besonders auf die Ausstattungsunterschiede zwischen dem KPZ und den regulären Stationszimmern zurückzuführen. Dieses Phänomen stand im allgemeinen Kontext der Versorgungsstrukturerweiterung für Kinder in der Palliativversorgung an der UMG und dem besonderen Augenmerk, dass auf die Integration der Familie des erkrankten Kindes ins Versorgungskonzept gelegt wird. Das Gefühl einer entstehenden „Klassengesellschaft“ wurde verursacht durch organisatorische Schwierigkeiten bei der Realisierung des KPZs, wie beispielsweise Beeinträchtigungen der Stationsroutine durch Baumaßnahmen und das Gefühl eines personellen Ressourcenmangels im Pflegebereich, der eine erfolgreiche Arbeit im KPZ subjektiv erschwerte. Zusätzlich bestanden bei vielen Mitarbeiten Unklarheiten und Unsicherheiten bezüglich des eigenen Aufgaben- und Verantwortungsbereiches. Die gefühlte Entstehung der „Klassengesellschaft“ hatte eine ablehnende Haltung vieler Mitarbeiter gegenüber dem Projekt KPZ zur Folge, was sich in einem distanzierten Verhalten äußerte. Bedingt wurde dieses distanzierte Benehmen dadurch, dass sich viele Mitarbeiter vom Entstehungsprozess des KPZs ausgeschlossen und nicht ausreichend über die Neuerungen informiert fühlten. Zusätzlich bestand die Befürchtung, zukünftig häufiger mit sterbenden Patienten konfrontiert zu sein, was als emotionale Herausforderung gesehen wurde. Hinzu kamen Konflikte unter den Mitarbeitern, die die Zusammenarbeit belasteten konnten. Als Konsequenz aus der ablehnenden Haltung des Personals gegenüber dem KPZ ergab sich eine Überforderung und Selbstunsicherheit. Einige sahen jedoch trotz der Herausforderungen, die eine Distanzierung vom Projekt verursacht hatten, im KPZ eine Chance zur persönlichen und beruflichen Kompetenzerweiterung.

\subsection{Kernkategorie: Durch KPZ entsteht „Klassengesellschaft“ auf der Station}

Zu Beginn der Studie wurde seitens der Arbeitsgruppe erwartet, dass besonders die durch das Kinderpalliativzimmer nahende Präsenz von Sterben und Tod einen großen Einfluss auf das Erleben der Mitarbeiter haben würde. Im Studienverlauf zeigte sich jedoch, dass viele strukturelle und organisatorische Herausforderungen die Entstehung des KPZ begleiteten und sich negativ auf das Erleben des Implementierungsprozesses auswirkten. In den erhobenen Daten hatte diese Thematik eine starke Präsenz, sodass die meisten der im Auswertungsprozess entwickelten Kategorien sich auf diese strukturellen Probleme 
beziehen. Zwar wurde deutlich, dass auch die Präsenz von Sterben und Tod, welche durch das KPZ gegeben ist, das Erleben beeinflusst, doch in den Erzählungen wurden diese deutlich von den strukturellen Schwierigkeiten überlagert.

Während der systematischen Verdichtung der Daten im Auswertungsprozess stellte sich heraus, dass es sich bei dieser Kategorie um die Kernkategorie und somit auch um das Kernphänomen im Bereich des Erlebens der Neugründung des KZP auf der Normalstation (vgl. Kapitel 4.4.3 Selektives Kodieren) handelte. Bemerkenswert ist, dass der Begriff der Klassengesellschaft in einem Interview explizit genannt wurde. Folgendes Zitat führte das Konzept „Klassengesellschaft auf der eigenen Station“ ein und vermag das Phänomen beschreibend einzuleiten:

\begin{abstract}
„dann zum Beispiel, auch mit dem Balkon? das fanden wir alle ist ja ganz nett aber wir dürfen ja eigentlich die Türen nicht aufschließen auf den Balkon was machen=sagen wir Eltern im Sommer die nicht in diesem Palliativizimmer sind warum dürfen die nicht da raus aber (2) die anderen schon? des ist (2) teilweise dann so en bisschen so ne Klassengesellschaft auf der eigenen Station wo wir uns nicht so ganz. mit anfreunden konnten"(KPZ 02, Z. 120-125)
\end{abstract}

\title{
5.4 Kontext der Kernkategorie
}

Es stellte sich heraus, dass durch die bauliche Gestaltung eine räumliche Distanz zwischen dem KPZ und dem Rest der Station entstand. Beim KPZ handelt es sich um das Eckzimmer einer u-förmigen Station. Ein offener Zugang bzw. eine direkte Einsicht in das Zimmer ist nicht gegeben, da hinter der Zimmertür der Elternbereich liegt. Die Tür ist mit einem Innenrollo versehen, damit die Eltern mehr Privatsphäre haben und keine direkte Einsicht vom Flur in das Zimmer möglich ist. Die anderen Stationszimmer haben ebenfalls Scheiben in der Tür, welche aber nicht durch ein vergleichbares Innenrollo verschlossen werden können. Ein Einblick in die Zimmer vom Flur aus ist so gegeben. Dies dient dazu, dass dem Personal ein Einblick ins Patientenzimmer möglich ist, hat aber gleichzeitig zur Folge, dass Begleitpersonen weniger Privatsphäre haben. Das KPZ muss auch für einen Badezimmerbesuch nicht verlassen werden, weil ein eigenes Bad vorhanden ist. Damit unterscheidet es sich zum einen von den anderen Zimmern der Station (vgl. Kapitel 3.6 Das Kinderpalliativzentrum Göttingen mit Kinderpalliativzimmer), zum anderen findet eine räumliche Abgrenzung von der restlichen Station statt. Diese ,Abgeschlossenheit ${ }^{`}$ kann von den Beteiligten positiv und negativ wahrgenommen werden: Auf der einen Seite können Familien und Patienten in schwierigen Phasen der Erkrankung und in der 
Sterbephase eine gewisse Privatsphäre genießen, weil sie vom ,Stationstrubel' räumlich entfernt sind. Auf der anderen Seite verursacht diese bauliche Gestaltung eine Abtrennung des Zimmers vom Stationsleben, welche wiederum eine emotionale Distanzierung mit sich bringen oder begünstigen kann. Im folgenden Zitat wird das KPZ mit einer Insel verglichen. Dieser Begriff kann zum einen emotional sehr positiv assoziiert sein, z. B. Insel - Urlaub - Loslösung vom Alltag. Andererseits ist eine Insel durch das sie umgebende Wasser auch isoliert, sodass kein problemloses Hin- und Fortkommen möglich ist:

„das is so es is schon so nen bisschen denk ich dadurch dass das so leicht abgeschottet ist es is ja schon nen bisschen wie so ne Insel einfach" (KPZ 18, Z. 470-471)

Das Kernphänomen ist außerdem in zwei Kontextkategorien eingebettet, die allgemeine Vorbedingungen und Ausprägungen des Phänomens beschreiben. Dies sind zum einen die Kategorie „Einberiehung der Familie ins Versorgungskonzept" und zum anderen „Aufbau UMG eigener Versorgungsstrukturen für Palliativkinder". Diese beiden Kategorien werden im Folgenden näher erläutert.

\subsubsection{Einbeziehung der Familie ins Versorgungskonzept}

In der Kinderpalliativmedizin ist neben der Behandlung der Patienten auch die Einbeziehung der Familie in das Versorgungskonzept von großer Bedeutung (vgl. Definition der WHO Kapitel 3 Stand der Forschung). Diese Auffassung teilten auch die interviewten Mitarbeiter der UMG. Auch schon vor der Entstehung des KPZ wurde die Integration der Familie in das Versorgungskonzept von den Mitarbeitern berücksichtigt. Durch das KPZ und dem Anspruch, der an Palliativmedizin für Kinder gestellt wird (vgl. Kapitel 3.1 Palliativmedizin im Kindesalter), stieg die Bedeutung der Familienbetreuung. Diese Kategorie wurde besonders von Mitarbeitern der psychosozialen Berufsgruppen geprägt, zu deren besonderer Aufgabe die Familienunterstützung gehört. In der konkreten Arbeit zeigte sich, dass die Existenz des KPZ die Arbeit bzw. Kontaktaufnahme des Personals mit betroffenen Familien erleichterte, beispielsweise die von manchen Mitarbeitern des Kinderpalliativzentrums, welche die Familien auch in organisatorischen Fragen berieten und ihnen beim Übergang in die ambulante Palliativversorgung zur Seite standen.

Es zeigte sich, dass viele Mitarbeiter großes Verständnis für die schwierige Situation hatten, in der sich Eltern von Kindern mit lebensbedrohlichen und lebenslimitierenden Erkrankungen befinden: 
„also überfordert- ich meine ich glaube mit nem sterbenden Kind is eigentlich fast jeder überfordert in Anfübrungsstrichen" (KPZ 12, Z. 484-486)

Die Mitarbeiter zeigten sich sehr empathisch für die Ausnahmesituation, in der sich die betroffenen Familien befinden und waren sich bewusst, dass die Einbeziehung der Familie in die Versorgung notwendig ist. Besonders betraf auch dies die befragten Mitarbeiter aus dem psychosozialen und ambulanten Bereich ${ }^{5}$.

Viele der befragten Mitarbeiter hatten den Eindruck, dass eine Entlastung der Familien durch die Mitglieder des Kinderpalliativ-Teams und die Stationsmitarbeiter stattfand. Diese dargebotenen Unterstützungsangebote der Mitarbeiter waren sehr vielschichtig: Die Familienunterstützung begann mit einem Beistand bei der Diagnosevermittlung. Viele Eltern standen psychisch unter hohem Druck, wenn sie eine schlechte Diagnose vom behandelnden Arzt oder der Ärztin übermittelt bekamen. Sie hatten große Angst um ihr Kind und konnten in dieser Situation die überbrachten Informationen gar nicht ,begreifen'. Dies wurde in der im folgenden Zitat beschriebenen Szene veranschaulicht. Hier konnte ein psychosozialer Beistand unterstützend wirken:

$$
\begin{aligned}
& \text { „und da stellt man fest dass die gar nichts gehört haben also die wissen welche Gardiune } \\
& \text { bing die wissen ob die Sonne da war oder der Regen aber die ganzen Inhalte der geht ganz } \\
& \text { viel verloren die beschäftigt nur stirbt mein Kind oder nich und dann nochmal da } \\
& \text { bin₹uweisen, da is gar nichts angekommen ibr müsst die Therapie das müsst ibr noch } \\
& \text { mal erklären" (KPZ 04, Z. 143-147) }
\end{aligned}
$$

Weiterhin waren praktische Unterstützungen der Familien während der Krankheitsphase, wie z. B. sozialrechtliche Beratung und die Unterstützung bei der Organisation der ambulanten Betreuung, von zentraler Bedeutung. Das Kinderpalliativ-Team der UMG steht den Eltern in der ambulanten Betreuung 24 Stunden telefonisch zur Verfügung, so dass auch in Notsituationen ein Ansprechpartner verfügbar ist. Dies soll den Eltern Halt und Sicherheit vermitteln. Bei stationärer Versorgung des Kindes versuchte das Personal, auch den Eltern Freiräume zu schaffen, indem sie z. B. das Kind betreuten, damit die Eltern beispielsweise einen Kaffee trinken gehen oder Zeit mit eventuell vorhandenen Geschwisterkindern verbringen konnten. Die Unterstützung bestand auch über den Tod des Kindes hinaus, z. B. in Form von Trauerbegleitung und Elternnachsorge:

\footnotetext{
5 An dieser Stelle wird kein Anspruch auf Generalisierbarkeit erhoben. Beim vorliegenden Sample ließ sich diese Beobachtung machen. Andere Stichproben mögen einen divergenten Schluss zulassen.
} 
„,diese Begleitung geht für mich auch über den Tod hinaus, aber trotzdem muss man wissen das geht nich bis ultimo aber, und man merkt das auch es gibt dann auch Eltern die irgendwann sagen ja jetz̧t is gut so nach dem Motto jetz̧ wollen wir mal andere , Dinge haben also so am Anfang ist es oft so dass die noch ganz oft auf die Stationen kommen aber irgendwann kommen sie dann nich mehr weil dann is einfach was anders angesagt und das is ja auch gut so" (KPZ 13, Z. 495-501)

Einige Mitarbeiter bemühten sich besonders darum, ein Vertrauensverhältnis zu den Patienten und den Familien aufzubauen, was die Zusammenarbeit erleichtern konnte. Besonders schwierige Gespräche z. B. über Entscheidungen am Lebensende des Kindes, konnten, nach Einschätzung der Mitarbeiter, besser geführt werden, wenn sich zuvor ein Vertrauensverhältnis zwischen Mitarbeitern und Familien gebildet hatte:

„weil man erstmal sich kennenlernen muss das sind ja Gespräche das sollte man ja erst bei solchen- in sone Gesprächstiefe kommt man ja erst wenn man nen paar Gespräche gefübrt hat , und sich nen bisschen vertraut und nen bisschen kennengelernt hat" (KPZ 12, Z. 608-611)

Es zeigte sich aber auch, dass einige Mitarbeiter offenbar ihre eigenen Wahrnehmungen, v. a. das KPZ betreffend, auf Eltern übertrugen. So hielten sie das KPZ für einen Ort, an dem man das Sterben seines Kindes als angenehmer empfinden könne, als dies in einem regulären Zimmer der Fall wäre und mutmaßten, dass es betroffenen Eltern genauso ergehen müsse. $\mathrm{Ob}$ dies wirklich der Fall ist, könnte jedoch nur in Gesprächen mit betroffenen Eltern erfahren werden:

„wenn ich mir vorstelle in diesem Zimmer da ist es zu Ende gegangen dann würde ich irgendwie sagen ja okay das war aber irgendwie en Ort, wo man, trotz dieser ja widrigen Umstände da irgendwas positives damit verbindet wo man sagt okay ja" (KPZ 15, Z. 712-715)

Für eine gute Familienunterstützung ist die Wahrung eines professionellen Abstandes von besonderer Bedeutung. Darauf versuchten viele der Interviewten besonders zu achten:

„ich muss schon gucken ich muss schon auch den professionellen Abstand haben aber ich darf nich also es darf jetżt nich so sein dass ich so drauf bin dass die Eltern mich trösten das geht gar nich das geht auf gar keinen Fall" (KPZ 13, Z. 520-523)

Bei allem Verständnis, welches die Mitarbeiter für die Ausnahmesituation der Familien hatten und der Unterstützung, die sie den Familien gaben, mussten viele auch feststellen, dass der Umgang mit den Familien z. T. eine große Hürde darstellte, die sie an die eigene 
Belastungsgrenze bringen konnte. Die Mitarbeiter nahmen bei den Eltern verschiedene Bewältigungsstrategien wahr und es fiel ihnen teilweise schwer, für alle diese Bewältigungsmechanismen Verständnis aufzubringen. Daraus konnten Konflikte zwischen Familien und Mitarbeitern resultieren. So konnte es sein, dass einzelne Mitarbeiter als Vermittler zwischen Eltern und Kollegen auftreten mussten:

„manchmal Eltern einfach so aggressiv nach außen wirken aber unter der Aggression is ja oft ne ziemliche Verzweiflung oder Angst und dass man diese zweiten Aspekt noch mal darstellt auch bei Besprechungen oder Visiten um so nen bisschen wieder Einvernehmen oder eine wohlwollende Haltung- es gibt Leute die verbalten sich einfach unverschämt und das is extrem schwierig für die Schwestern auszuhalten ne und kann man versuchen mit der zweiten Seite noch mal zu punkten indem man sagt ja die die können das im Moment nich besser ne das is deren Form der Verarbeitung das müsst ibr jetzt nich persöölich nehmen und das is ja immer genau das Schwierige" (KPZ 04, Z. 561-569)

Manche Mitarbeiter zeigten sich sehr empathisch für die besondere Rolle von Geschwisterkindern. Auch für diese - so wurde es von den Interviewpartnern antizipiert ist die Krankheit bzw. der Tod der Schwester oder des Bruders eine große emotionale Belastung. Den Mitarbeitern stellte sich die Frage, inwieweit die gesunden Geschwisterkinder Anteil nehmen sollten am Schicksal ihres erkrankten Bruders oder ihrer Schwester. Der Geschwisterversorgung wird deshalb auch von Seiten des Personals viel Aufmerksamkeit geschenkt, weil diese als besonders unterstützungsbedürftig wahrgenommen wurden:

„und dann gibt es Geschwisterkinder die ja auch mitbetreut werden sollen wollen können und ähm was uns auch gan₹, gan₹, wichtig is dass die nich binten runterfallen, und auch da gibt es einfach Gespräche Gespräche mit den Geschwisterkindern auch das is absolut wichtig weil die müssen teilweise eh immer zurückstecken weil das immer um das erkrankte Kind geht" (KPZ 13, Z. 283-288)

Anders verhielt es sich mit der Empathie gegenüber betroffenen Eltern. Diese wirkte teilweise etwas geringer ausgeprägt, als jene für betroffene Geschwisterkinder. Möglicherweise deshalb, weil die Eltern als weniger hilfsbedürftig wahrgenommen wurden und weil man bei ihnen einen, reiferen` Umgang mit der Situation erwartete. Dies zeigt sich z. B. in der verwendeten Begrifflichkeit im folgenden Zitat („Scheuklappen“) und der distanzierten Sprache. Das Verhalten der Eltern in der emotionalen Ausnahmesituation wurde hier durch die interviewte Person als fehlende Bereitschaft der Eltern, sich mit dem Tod ihres Kindes auseinander zu setzen, gedeutet: 
„dass die Eltern dann so nen bisschen diese Scheuklappen haben und es vielleicht noch gar nicht seehen wollen und dass das vielleicht jetzt irgendwann mal passiert wird werden viele klammern sich ja doch lange dran"(KPZ 18, Z. 371-374)

Besonders für die Mitarbeiter der psychosozialen Berufsgruppen rückte, in dieser Studie, die Familienunterstützung in den Vordergrund:

„mein Schwerpunkt liegt eigentlich darauf wie die Eltern zu unterstïtzen und auch wie möglicherweise Geschwister zu unterstütren wenn sie da sind wenn sie das möchten, weil die müssten ja sozusagen den Weg mitgehen und brauchen auch dementsprechend, ähm, eine Unterstützung um das ganze irgendwie auch bewältigen zu können" (KPZ 06, Z. 100-104)

\subsubsection{Aufbau UMG-eigener Versorgungsstrukturen für Palliativkinder}

Die UMG hatte eigene Versorgungsstrukturen für lebenslimitierend und lebensbedrohlich erkrankte Kinder und Jugendliche aufgebaut und war dabei, diese zu verbessern und zu erweitern. Das Kinderpalliativzentrum bietet mit einem eigenen Team seine Unterstützung in der SAPV an. Daneben stellte das KPZ eine Erweiterung der stationären Versorgungsmöglichkeiten dar.

Eine stationäre Versorgung des Kindes kann dann notwendig werden, wenn in schwierigen Phasen der Erkrankung und am Lebensende Symptomverschlechterung und Unkontrollierbarkeit der Symptome die Situation für die Familien zu Hause schwer handhabbar machten. Einige Mitarbeiter hatten den Eindruck, dass viele Familien und auch Kinder durch die Symptome verängstigt wurden und nahmen an, dass manche Situationen (z. B. zerebrale Krampfanfälle, Atemnot) von der Familie nicht schnell und effizient genug zu Hause bewältigt werden könne. Eine stationäre Betreuung schien sich beruhigend auf die Familien auszuwirken, da rund um die Uhr kompetente medizinische Versorgung zur Verfügung stand. Dem Personal schien es, als könnten sie den Familien ein Gefühl von Sicherheit vermitteln, auch weil die Eltern und Patienten die Möglichkeit hatten, die Verantwortung an sie abzugeben:

„die Patientin hatte furchtbare Angst die wollte nicht die wollte nicht alleine sie wollte wobl nich Zuhause sein sie wollte unbedingt in die Klinik, also das war einmal so immer dann wenn sie Angst bekommen hat wollte sie schnell von Zuhause weg und wollte in die Klinike weil sie sich da sicherer gefüblt hat" (KPZ 15, Z. 211-214) 
Gründe für den Aufbau dieser eigenen Versorgungsstrukturen waren zunächst die vorher fehlenden stationären Rahmenstrukturen für die Versorgung schwerstkranker Kinder in Göttingen:

„wo wir früher gesagt haben da können wir jetə̧t nich mehr helfen wir haben dafür keine Räumlichkeiten wir können solche Patienten auch nicht so lange, stationär aufnehmen sondern dafür gibts jetz̨t andere Institutionen wie zum Beispiel ein Hospiz." (KPZ 15, Z. 586-589)

Es fehlten räumliche und personelle Strukturen, die eine Versorgung der Kinder ermöglicht hätten, und es mussten in der Vergangenheit andere Versorgungsmöglichkeiten für die Kinder gesucht werden. So wurde beispielsweise die Unterbringung in einem Hospiz oder eine ambulante Betreuung des Kindes zu Hause sichergestellt. Doch nicht immer konnten passende Lösungen gefunden werden. So verstarb eine Jugendliche/ein Jugendlicher auf der Palliativstation für Erwachsene, weil die Versorgung zu Hause oder im Hospiz keine Alternativen darstellten und die Patientin/ der Patient aus dem ,Trubel' der Kinderstation raus wollte. Es zeigte sich, dass der Bedarf für eine stationäre Versorgungsstruktur für Kinder in einer Palliativsituation im Raum Göttingen bestand:

„weil wir einfach die Erfahrung gemacht haben dass es immer mal wieder Kinder gibt die nich unbedingt Zubause sterben wollen und von daher is das guut dass es sonen Zimmer gibt, aber das heißt ja nich dass wir trotzdem bestrebt sind halt dass wenn die Kinder das möchten dass wir sie Zuhause weiter begleiten, und dass es son Zimmer gibt hier in der Uniklinik denk ich is schon gut gar keine Frage" (KPZ 13, Z. 15-20)

Es wurde dadurch eine zusätzliche Versorgungsmöglichkeit - neben Hospiz, Zuhause und stationärer Versorgung auf einer Normal- oder Intensivstation - geschaffen, wo der Familie die medizinische Sicherheit eines Krankenhauses geboten werden kann, aber eine annehmlichere und privatere Atmosphäre besteht als in einem regulären Krankenhauszimmer:

„aber wenn es Zubause nich geht und wenn die Familie da überfordert ist und das nicht mehr aushält oder dem Kind es tatsächlich auch zu schlecht geht ja (2) es is Gott sei Dank möglich dass die auch in der-können die ja sowieso in der Klinik versterben also das konnten sie ja auch schon bevors das Kinderpalliativizimmer gab ja das is aber jetzt nochmal eine erweiterte Möglichkeit mit wirklich schönerer Atmosphäre ja als in einem normaalen Krankeñimmer ja “ (KPZ 08, Z. 343-348) 
Das Personal sah die Vorteile des KPZs darin, dass für ein sterbendes Kind und seine Familie eine Versorgungsmöglichkeit geschaffen wurde, wo sie eine größere Rückzugsmöglichkeit hatten. Trotz dieser privaten Atmosphäre ist eine medizinische Versorgung gegeben. Doch waren sich noch nicht alle Mitarbeiter sicher, ob der Bedarf für das Kinderpalliativzimmer insoweit bestand, dass es durchgehend mit „Palliativpatienten“6 belegt werden könnte. Auch stellten sie sich die Frage, ob nicht viele der Kinder, die im Krankenhaus versterben, dies auf der Intensivstation tun werden, weil die Eltern eine intensivmedizinische Versorgung des Kindes wünschen würden. Zudem dürfen im KPZ bspw. keine dauerhaft beatmeten Kinder untergebracht werden, so dass diese nach wie vor auf der Kinderintensivstation versorgt werden würden. Viele Mitarbeiter waren deshalb noch unsicher, was den Bedarf und die Entstehungsgründe vor allem mit Bezug auf den vermuteten Vorteil für die Patienten betrafen, und gaben sich abwartend. Diese Unsicherheit lässt sich an der Formulierung ,ich glaub ähm“ festmachen:

$$
\begin{aligned}
& \text { „und deswegen fand ich das ne sehr gute Idee dass wir das geplant haben und dann } \\
& \text { überlegt haben wos so stattinden kann wos gebaut werden kann ja und ich glaub ähm das } \\
& \text { is schon nen großer Gewinn für die Patienten" (KPZ 12, Z. 19-22) }
\end{aligned}
$$

Neben dem Kinderpalliativzimmer für die stationäre palliativmedizinische Versorgung baute die UMG auch den ambulanten Palliativdienst mit der spezialisierten ambulanten Palliativversorgung für Kinder weiter aus. Neu hinzugekommen war im Januar 2015 die zusätzliche Übernahme der pflegerischen Versorgung durch Gesundheits- und Kinderkrankenpfleger der Klinik für Kinder- und Jugendmedizin. Damit stand den Familien in der SAPV rund um die Uhr ein ärztlicher und pflegerischer Palliativdienst unterstützend zur Seite. Das Kinderpalliativzentrum Göttingen übernahm auch in der Vergangenheit schon eine ambulante Betreuung und Beratung, jedoch nicht im pflegerischen Bereich durch Mitarbeiter der UMG.

Gründe für diesen Ausbau des ambulanten Palliativdienstes war zum einen das Bestreben, die ambulante Versorgung von Kindern weiter auszubauen, zum anderen wurde der Vertrag zwischen dem ambulanten Kinderkrankenpflegedienst $\left(\right.$ KIMBU $\left.^{7}\right)$ - welcher vorher die ambulante pflegerische Betreuung der Kinder übernommen hatte - und der Klinik für

\footnotetext{
${ }^{6}$ Hier sei angeführt, dass der Begriff „Palliativpatient“ von den Mitarbeitern nicht immer im Sinne der im Kapitel 1 eingeführten Definitionen verwendet wird. Für viele Mitarbeiter ist ein „Palliativpatient“ nur der, welcher unmittelbar im Sterben liegt. „Palliativpatient“ wird deshalb in Anführungszeichen dargestellt, um zu verdeutlichen, dass es sich um die Definition von Mitarbeitern handelt. Auf die Problematik der Begriffsunsicherheit wird in Kapitel 6.6 noch näher eingegangen.

${ }^{7}$ KIMBU - Häusliche Kinderkrankenpflege Göttingen gemeinnützige GmbH
} 
Kinder- und Jugendmedizin beendet. Somit musste eine Versorgungsalternative geschaffen werden:

„genau also dies ärztliche Team is von jeher von der Klinik, gestellt worden und es is bis 2014 von der Kimbu pflegerisch übernommen worden und da is der Vertrag nich verlängert worden aus welchen Gründen auch immer ((lacbt)) "(KPZ 17, Z. 167-170)

Dieser pflegerische Palliativdienst steht betroffenen Familien 24 Stunden am Tag zur Verfügung. Die Pflegekraft, welche Rufdienst hat, nimmt den Anruf entgegen und stellt die erste Anlaufstelle dar. Am Telefon muss dann von ihm/ihr entschieden werden, ob das Problem telefonisch oder persönlich von ihr/ihm vor Ort gelöst werden kann oder ob eine Ärztin oder ein Arzt zu einem Hausbesuch hinzugezogen werden sollte.

Dieser ambulanten Arbeit blickten einzelne Mitarbeiter positiv entgegen, weil hier der einzelne Mitarbeiter mehr selbstbestimmtes Handeln praktizieren kann, als es bei der Arbeit in der Klinik der Fall ist:

,ja , ja es is ja nen ganz, anderes Arbeiten als in der Klinik. und auch und ich finde man hat ein bisschen mehr Eigenverantwortung "(KPZ 17, Z. 248-249)

\subsection{Ursächliche Bedingungen der Kernkategorie}

Durch die großen baulichen und gestalterischen Unterschiede zwischen dem KPZ und den anderen Zimmern der Station entstand das Gefühl einer „Klassengesellschaft“ auf der Station. Eine Unterbringung im KPZ ist mit besonderen Privilegien verbunden. Es gibt im KPZ einen Balkonzugang, ein eigenes Bad und einen Bereich, in dem mehrere Begleitpersonen untergebracht werden können (vgl. Kapitel 3.6 Das Kinderpalliativzentrum Göttingen mit Kinderpalliativzimmer). Dies könnte zur Konsequenz haben, dass andere Patienten und Angehörige mit Neid reagieren würden und ebenfalls im KPZ untergebracht werden wollen. Auf diesen Aspekt wird in der Diskussion (Kapitel 6.1.1 Die Antizipation von Neid) näher eingegangen. Besonderes Konfliktpotential bietet, der Einschätzung vieler Mitarbeiter zur Folge, die Balkonsituation. In der Abteilung Neuropädiatrie ist ein Betreten der Balkone nicht gestattet, da die vorhandenen Geländer als nicht absturzsicher gelten. Die Balkontüren der Stationszimmer lassen sich deshalb nicht komplett öffnen. Dies hat, aufgrund der Ausrichtung der Station nach Süden, im Sommer eine große Hitzeentwicklung in den Patientenzimmern zur Folge. Der Geländerbereich am KPZ wurde im Rahmen der Umbauarbeiten verändert und absturzsicher gestaltet. Zusätzlich erhielt der Balkon einen Holzfußboden und wurde 
bepflanzt. Diese Gegebenheiten lassen unter anderem eine Unterbringung im KPZ erstrebenswert erscheinen:

„naja einfach weil es ein tolles Zimmer ist? du darfst da auf den Balkon woanders darf man nicht auf den Balkon ne man hat da nen Fernseher drin woanders hat man den nicht und man hat ein tolles Bad irgendwie wo man duschen kann und es ist einfach ein seebr komfortables Zimmer da dürfen da darf ne zweite Begleitperson mit schlafen in andern Zimmern dürfen sie es nicht, so oder so dürften sie aber ist zu eng gebt nicht" (KPZ 07, Z. 1295-1300)

Es entstand deshalb unter den Mitarbeitern das Gefühl einer sich bildenden „Klassengesellschaft“ zwischen den Patienten, die die Vorteile im KPZ nutzen können und jenen, die in den regulären Stationszimmern untergebracht sind. Zu bedenken ist hierbei, dass das KPZ nicht durchgängig mit „Palliativpatienten“ belegt ist. Ist kein „Palliativkind“ in der Kinderklinik aufgenommen, wird das KPZ mit ,Nicht-Palliativkindern“ belegt. Es ist geplant, dass dann das jeweils am schwersten erkrankte Kind der Station im KPZ untergebracht wird. Eine objektive Bewertung der Schwere einer Erkrankung ist sehr schwierig, sodass die Mitarbeiter Uneinigkeit bei der KPZ-Belegung mit ,NichtPalliativkindern` erwarteten.

Auch zwischen den Mitarbeitern fand insofern eine Aufteilung statt, als jene eine Sonderstellung im Team erhielten, die besondere Qualifikationen (z. B. Pädiatrische Palliative Care Weiterbildung) hatten, zusätzliche Aufgaben übernahmen oder Mitglied im Kinderpalliativzentrum waren. Sie verfügten über ein umfangreicheres fachliches Wissen im Bereich Kinderpalliativmedizin und konnten von jenen Kollegen, denen solch ein Vorwissen fehlte, als fachliche Unterstützung wahrgenommen werden.

Daneben begünstigten zwei weitere Kategorien als ursächliche Bedingungen die gefühlte Entstehung der „Klassengesellschaft““

\subsubsection{KPZ als organisatorische Herausforderung}

In den Interviews konnten häufig organisatorische Herausforderungen identifiziert werden, die durch das KPZ verursacht wurden. Sie sind ursächlich für den Eindruck der sich bildenden „Klassengesellschaft“ auf der Station.

Die Mitarbeiter empfanden einen generellen stationären Ressourcenmangel in der Klinik, der räumliche, strukturelle und personelle Ressourcen betraf. In diesem Kontext stellte die Einführung des KPZs eine besondere Herausforderung dar: 
„,und man auch nen bisschen naja Zeit für Gespräche und so brauch die is nich da, die is definitiv nich da wenn es im ganz normalen Tagesgeschehen is, und dann noch ne Kollegin zum Beispiel krank is dann is es definitiv nich dann is man wirklich am Rennen also dann is man froh wenn kein Patient verloren geht irgendwie im Laufe des Vormittags das is tatsächlich so (3) also es gibt auch rubigere Dienste aber also bäufig erlebe ich es tatsächlich dass man so seiner Arbeit binterber rennt" (KPZ 10, Z. 470-476)

Die Stationsroutine wurde durch Baumaßnahmen wie Baulärm, Dreck und der vorübergehenden Reduzierung der Stationskapazität um zwei Patientenbetten beeinflusst. Da das KPZ aus einem ehemaligen Doppelzimmer entstand, war es während der Umbauarbeiten notwendig, dass häufiger Patientenverlegungen auf andere Stationen vorgenommen werden mussten.

Über die Baumaßnahmen hinaus verstärkte das KPZ den vorhandenen räumlichen Ressourcenmangel, da nach seiner Fertigstellung der Station insgesamt ein Bett weniger zur Verfügung stand. An dieser Stelle sei angemerkt, dass im KPZ zwar Anschlüsse für zwei Patientenbetten vorhanden sind, eine Doppelbelegung jedoch nicht standardmäßig geplant ist - zumindest dann nicht, wenn das KPZ mit einem Palliativpatienten belegt ist. Zusätzlich steigt - nach Meinung einiger Befragten - voraussichtlich die Arbeitsbelastung des Personals durch ein pflegeaufwendigeres Kind. Da zu einer palliativmedizinischen Versorgung neben der Beachtung der physischen, psychischen und spirituellen Bedürfnisse des Kindes auch die Versorgung der Familie von Bedeutung ist (vgl. Definition der WHO Kapitel 3 Stand der Forschung), sahen viele Mitarbeiter besonders dort eine Arbeitszunahme:

„also so wie ich mir des in meiner Vorstellung vorstelle muss des fast eine 1 zu 1 Betreunng sein weil dieses Kind wird sehr viel Aufmerksamkeit brauchen und nicht nurdas Kind sondern auch die Eltern die ja dabei sind" (KPZ 02, Z. 548-550)

Organisatorische Regelungen der Station (z. B. Ablauf der Visite, Pflegedokumentation) müssten - nach Meinung einiger Mitarbeiter - überdacht und angepasst werden. Manche Mitarbeiter befürchteten, dass die vorhandenen Möglichkeiten eine gute palliativmedizinische Versorgung des Patienten schwierig machen könnten: 
„und ich glaub das sind auch viele Ängste eben dass man denkt naja jețt hat man hier son Zimmer und mehr Arbeit aber irgendwie gar keine Zeit dafür=das man man will ja seine Arbeit auch gut machen man will ja nach Hause gehen mit dem mit dem Wissen ich hab das gut gemacht und ich bin ich hab auch alles mitgekriegt von meinen Patienten und bin nich nur so von $A$ nach $B$ gerannt und hab nur die Hälfte bemerkt man will ja guut arbeiten (4) das geht eben manchmal nur bedingt wenn man sehr knapp besetzt is das is schwierig"(KPZ 10, Z. 477-484)

Ein personeller Ressourcenmangel schien vor allem in der Pflege zu bestehen, den man abzumildern versuchte. Zum einen dadurch, dass seitens der Klinikleitung eine zusätzliche Stelle geschaffen wurde, durch die sich der Betreuungsschlüssel in der Spätschicht verbessern sollte. Zum anderen dadurch, dass einige anfallende Aufgaben, z. B. Grundpflege des palliativ versorgten Kindes, durch die anwesenden Eltern übernommen wurden:

„und die Station selbst hat glaub ich eine Pflegekraft mehr bekommen, zur Versorgung dieser Patienten" (KPZ 12, Z. 250-251)

„dadurch das wir in der Kinderkrankenpflege sind und bäufig Eltern mit dabei sind, fällt zum Beispiel sehr häufig die Pflege weg. mittlerweile. so Körperpflege wickeln solche Sachen machen wir (3) mittlerweile seltener" (KPZ 02, Z. 380-382)

Diese Bestrebungen des Personals wurden durch allgemeine strukturelle Abläufe in der Kinderklinik beeinflusst. Darunter zusammengefasst sind allgemeine strukturelle Zusammenhänge, z. B. Stationsaufteilung, Personalverteilung und Behandlungsschwerpunkte einzelner Abteilungen:

„wobei man sagen muss die Stationen sind schon sehr stark verwooben also es gibt einige Schwestern die arbeiten regelhaft auf zwei Stationen und so" (KPZ 07, Z. 1175-1177)

Es zeigte sich in den Interviews, dass eine Kompensation der durch das KPZ verursachten Mehrarbeit vielen Mitarbeitern unmöglich erschien. Besonders die eine zusätzlich geschaffene Pflegestelle schien zum Ausgleich des gestiegenen Arbeitsaufwandes nicht auszureichend zu sein:

„dann bilft dann auch quasi eine zusätzliche Kraft nicht weil diese zusätz̧liche Kraft ist dafür da das man halt jetz̨t nicht mehr hauptsächlich ₹u zweit im Spätdienst ist sondern dann mit drei Pflegekräften was aber für den Nachtdienst und Frübdienst keinerlei Relevanz hat da bleibt die Personaldecke exakt genauso" (KPZ 01, Z. 118-122) 
Andere Stationen sahen im KPZ allerdings durchaus eine Möglichkeit zur Kompensation des eigenen Ressourcenmangels:

„wie gesagt ähm wir haben auch Kaparitätsprobleme deswegen is das für uns natürlich sehr schöön dass es da noch ne Ausweichmöglichkeit gibt die vorber nich da war" (KPZ 11, Z. 276-278)

Zusätzlich $\quad \mathrm{zu}$ den organisatorischen Herausforderungen spielen Abgrenzungsproblematiken des Aufgaben- und Verantwortungsbereiches eine große Rolle bei der Entstehung der gefühlten „Klassengesellschaft““.

\subsubsection{Abgrenzungsproblematik des Aufgaben- und Verantwortungsbereiches}

In der Analyse wurde deutlich, dass viele Mitarbeiter offenbar Abgrenzungsschwierigkeiten ihres eigenen Aufgaben- und Verantwortungsbereiches hatten. Für die Entstehung dieser Abgrenzungsproblematik gab es verschiedene Ursachen: Bei einigen Mitarbeitern bestand eine Unsicherheit und Unklarheit bezüglich der eigenen Rolle, die daraus resultierte, dass der individuelle Aufgabenbereich nicht ausreichend definiert schien, wobei unklar blieb, ob dieser nicht verstanden wurde oder durch die Vorgesetzten nicht eindeutig festgelegt und vorgegeben worden war. Dies betraf auch die eigene Rolle bei der zukünftigen Versorgung des Kindes im KPZ:

$$
\begin{aligned}
& \text { „was das betrifft noch nicht im Detail geklärt war ich hab natürlich mit der Kollegin auf } \\
& \text { der Station Kontakt aufgenommen und wir haben gesagt okay wir werden da jetət } \\
& \text { demnächst ins Gespräch gehen und nochmal genau gucken und vielleicht abhängig auch } \\
& \text { von der einzelnen Situation wie wir das handhaben ja vielleicht gibts auch keine } \\
& \text { generelle Lösung das weiß ich gar nich" (KPZ 08, Z. 593-598) }
\end{aligned}
$$

Neben dieser Unklarheit bezüglich der persönlichen Rolle schien auch eine Identifikation mit bestehenden Strukturen zu fehlen. Dies wird darin deutlich, dass die Schilderung der Strukturen sehr theoretisch klang. Eine mögliche Erklärung dafür ist, dass in der Wahrnehmung der Mitarbeiter die Strukturen von Vorgesetzten vorgegeben wurden und nicht selbst gewachsen waren. Dies schien auch die multiprofessionelle Zusammenarbeit zu betreffen, die durch das KPZ noch verstärkt wurde:

Also das is ja immer ganz schwierig wenn man sich auch nicht gegenseitig so gut kennt? (KPZ 11, Z. 228-229)

Manche Mitarbeiter reagierten mit einer klaren Abgrenzung von anderen Berufsgruppen. Diese Abgrenzung bzw. klare Absteckung des eigenen Kompetenzbereiches konnte auf ein 
gewisses Konkurrenzgefühl zwischen den einzelnen Berufsgruppen zurückgeführt werden, welches Konflikte auf der Station begünstigen konnte.

Es kam aber auch vor, dass einzelne Mitarbeiter Verantwortung abgaben. Dies war ein empfundener Vorteil der multiprofessionellen und stationsübergreifenden Arbeit. So müssen erkannte Probleme nicht alleine bewältigt werden, sondern konnten gemeinschaftlich gelöst werden. Der Einzelne trug daher nicht die alleinige Verantwortung für die getroffenen Entscheidungen und konnte sich so vielleicht auch besser vor Überforderung schützen:

„alleine würde ich das nich machen, das is halt einfach ne Entscheidung die man nich allein treffen kann dass is immer im Team "(KPZ 12, Z. 644-646)

Bedingt wurden diese Strategien der Mitarbeiter durch das Gefühl einer teilweise fehlenden Übereinstimmung von Arbeitsethos und Arbeitsrealität. Die Mitarbeiter hatten eigene Vorstellungen, wie die individuellen Aufgaben (z. B. Versorgung der Patienten) erledigt werden und der Stationsalltag strukturiert werden sollte, damit eine optimale Erledigung der Aufgaben möglich war (Arbeitsethos). Dem gegenüber standen die realen Arbeitsumstände (gefühlte personelle Unterbesetzung) und persönliche Schwächen, wie z. B. hektische Reaktionen, die auf fehlende Routine hindeuten und die die Arbeit beeinflussten (Arbeitsrealität). Dies hatte zur Folge, dass eigene Vorstellungen und eigenen Ansprüche an die Qualität der Arbeit teilweise an der Arbeitsrealität scheiterten und nicht umgesetzt werden konnten.

Durch die palliativmedizinischen Zusatzaufgaben, welche das KPZ mit sich brachte, hatten einige Mitarbeiter jedoch das Gefühl, dass es zu einer Erweiterung des Aufgaben- und Verantwortungsbereiches kam. Diese Vergrößerung des Kompetenzbereiches durch die geschaffenen Rahmenstrukturen nahmen sie positiv wahr, weil es ihnen Sicherheit vermittelte. Beispielsweise war ein Hausbesuch bei Patienten durch das Klinikpersonal früher ungewöhnlich. Das KPZ und besonders der Ausbau der ambulanten Versorgung gab nun jedoch einen neuen Rahmen vor:

„die Frage hat sich einfach nich gestellt dafür gab es keinen Raum es gab weder den Auftrag von meinem Arbeitgeber dazu noch hatte ich die entsprechende Ausbildung jemanden jetzt im zum Tode zu begleiten Zubause aber ich glaube das ist finde ich ne Bereicherung dass ich die , jetzt anstrebe diese Möglichkeit zumindest auch zu baben" (KPZ 15, Z. 726-730) 
Als weitere Konsequenz ergab sich jedoch bei einigen Mitarbeitern, dass der (gefühlte) Verantwortungsbereich ihren Aufgabenbereich überstieg. Bei der interpretativen Interviewauswertung wurde immer wieder deutlich, dass sich viele Mitarbeiter für Dinge verantwortlich fühlten, für die sie primär nicht zuständig waren. Daraus konnte das Problem entstehen, dass der Anspruch an die eigene Rolle bzw. Funktion höher war, als man sie erfüllen konnte und auch höher, als sie vorgesehen war:

„das mach ich da sag ich nicht das ist nicht meine Aufgabe das ist nicht meine Aufgabe aber ich mache das weil ich das einfach selbstverständlich empfinde" (KPZ 03, Z. 200202)

Besonders häufig fiel dieser Sachverhalt bei der Frage der Zimmerbelegung auf. Fast alle Mitarbeiter machten sich Gedanken, mit welchem Patienten das KPZ belegt werden sollte. Es musste entschieden werden, welches Kind im Palliativzimmer versorgt werden ,durfte, wenn kein Palliativpatient aktuell in der UMG stationär behandelt werden musste. Diese Aufgabe erschien den Mitarbeitern sehr anspruchsvoll. Welches Kind sollte dann die Möglichkeit bekommen, ins KPZ verlegt zu werden? Die Mitarbeiter bekamen das Gefühl, die eigenen Patienten in zwei Gruppen einzuteilen, was sie nicht nur ein eine Konfliktsituation brachte, sondern auch zur gefühlten Entstehung einer „Klassengesellschaft“ beitrug:

„die Priiooriitätensetzung ist halt sehr schwer ne welches Kind hat jetżt mehr Anspruch darauf dieses Palliativizimmer zu beanspruchen ein Kind was jetzt im Sterben liegt oder aber ein Kind was eine lebensverkürzende Erkrankung hat und der es trotzdem genauso schlecht geht. ne des ist dann glaub ich auch schwierig mit welchen Patienten belegen wir jetat dieses Kind ähh dieses Zimmer das ist halt ja schwierig was ich wir hatten da wie gesagt letzte Woche schon ziemlich Stress" (KPZ 01, Z. 59-66)

\subsection{Handlungsstrategien der Kernkategorie}

Als Reaktion auf die empfundene „Klassengesellschaft“ kam es bei einigen Mitarbeitern zu einer Abwehrhaltung, welche vor allem durch die ungleiche Ausstattung der Zimmer verursacht wurde: 
„da würd ich als Mutter auch durchdrehen wenn mein Kind bier krank im Sommer liegt bei 40 Grad im Zimmer vielleicht sogar noch Fieber hat selber schwer krank dann würd ich als Mutter auch sagen nö ich will auch in ein Palliativizimmer wo ich die Möglichkeit babe frische Luft zu schnappen also das sind so Begründungen die ich auch später nachvollriehen kann" (KPZ 01, Z. 75-80)

„man darf nirgends auf den Balkon nur auf diesem Zimmer das ist ne schreiende Ungerechtigkeit ist auch so . und da wirds natürlich Diskussioonen geben" (KPZ 07, Z. 1276-1278)

Hier zeigte sich, welch großen Stellenwert die Balkonsituation bei der Bewertung des Zimmers hatte. In fast allen Interviews kam sie zur Sprache. Die Mitarbeiter mutmaßten, dass diese Situation großes Konfliktpotential bieten und zu Unruhen auf der Station führen würde. Besonders das Pflegepersonal sah sich in der ,Schusslinie ${ }^{6}$ und befürchtete eine Konfrontation mit Beschwerden von Eltern, deren Kind nicht im KPZ untergebracht war. Manche Mitarbeiter wollten als Reaktion auf die „Klassengesellschaft“ besonders darauf achten, dass sie die Patienten des KPZs und die der anderen Zimmer gleich betreuten. Es zeigte sich die Tendenz, dass manche Mitarbeiter befürchten, den Patienten der regulären Stationszimmer würde zu wenig Aufmerksamkeit zuteilwerden. Sie wollten deshalb der Versorgung dieser Kinder besondere Beachtung schenken:

„es darf eben nicht passiern das dieses Kind wichtiger ist als die andern also nur weil es grade sehr schwer krank ist heißt es nicht das die andern Kinder die krank sind darunter leiden dürfen also das glaub ich muss man sich immer wieder ins Gedächtnis rufen das, es auch noch '14' andere Patienten gibt, die ne Betreunng brauchen (3)" (KPZ 02, Z. 564 568)

Viele Mitarbeiter hofften, dass die Gleichbehandlung der Stationspatienten eine Selbstverständlichkeit sein würde. In der Formulierung dieses Wunsches als Hoffnung zeigte sich aber, dass sie sich nicht völlig sicher waren, dass eine Gleichbehandlung eintreten würde:

„ich hoffe dass die Betreunng immer gleich gut is egal wie das Zimmer is" (KPZ 16, Z. 376-377)

Die Datenauswertung ergab, dass das Personal aus der gefühlten Entstehung einer „Klassengesellschaft“ zur Überwindung dieser konflikthaften Situation mit der Strategie einer distanzierten Haltung gegenüber dem KPZ reagierte. 


\subsubsection{Ablehnung des KPZs}

Infolge der organisatorischen Schwierigkeiten und der Abgrenzungsproblematik des Aufgaben- und Verantwortungsbereiches sowie der daraus resultierenden empfundenen Entstehung einer „Klassengesellschaft“ kam es augenscheinlich zu einer Abweisungsreaktion des Personals gegenüber dem KPZ. Diese diente möglicherweise der Reduktion einer inneren Konfliktsituation in Bezug auf die optimale und egalitäre Versorgung aller Patienten. Dabei war die Ablehnung stärker bei den Mitarbeitern zu beobachten, die in ihrer täglichen Arbeit mit dem KPZ konfrontiert waren, was diese Annahme der Begründung jedoch unterstützt. Sie sahen sich mit allen organisatorischen und emotionalen Herausforderungen konfrontiert, die die Initiierung des KPZs mit sich brachte. Mitarbeiter, die nicht unmittelbar mit dem Zimmer arbeiteten, zeigten in den Interviews weniger ausgeprägte Abwehrreaktionen, weil sie von den Bedingungen, die die Ablehnung verursachten, weniger bis gar nicht betroffen waren.

Zusätzlich zum neu errichteten KPZ sorgte ein Vorgesetztenwechsel im Pflegebereich für Unsicherheit beim Personal, obwohl dieser Wechsel zeitnah angekündigt worden war:

$$
\text { „gleichreitig wissen die Kolleginnen auch grad gar nicht auf welche Veränderungen sie sich }
$$
jetzt zuerst einlassen sollen" (KPZ 07, Z. 315-317)

Es zeigte sich außerdem, dass der Begriff ,Kinderpalliativzimmer ${ }^{\star}$ bzw. ,palliativ“ im Allgemeinen stigmatisiert $\mathrm{zu}$ sein scheint und ein abwehrendes Verhalten nach Konfrontation die Folge sein kann. Die Mitarbeiter selbst hatten mit einer Begriffsunsicherheit zu kämpfen. Zusätzlich mussten sie sich mit einer Verängstigung der Eltern durch die Begrifflichkeit auseinandersetzen. Manche Interviewpartner hatten den Eindruck, dass es Eltern gab, die eine Verlegung ins KPZ mit einem ,Todesurteil' für ihr Kind gleichzusetzen schienen:

„und (2) dann wurde halt den Eltern gesagt wir würden sie gerne in das Zimmer umlegen und dann hatten die Eltern gan₹,große Augen gemacht und ham gesagt aber 'warum denn', wir wolln da nicht rein und dann musste man den erstmal vermitteln das wir das nicht machen möchten um, sie in ein Palliativ₹immer zu legen sondern um sie in ein Zimmer zu legen wo die (2) Einrichtung viel besser sind wo wir sie besser pflegen können? wo mebr Möglichkeiten sind um sie zu mobilisiiern und solche Sachen aber des war $=$ des hat erstmal ein langes Gespräch erfordert um denen zu erläutern das, man ibr Kind da nicht binlegen möchte ₹um=um sie da sterben ₹u lassen=was ja alle denken" (KPZ 02, Z. 254-262) 
Diese Begriffsstigmatisierung stellte eine Herausforderung für das Personal dar:

„,ich fänds gan₹, furchtbar wenn da groß draußen dran stehen würde Palliatiivaimmer dann hätten die Patienten von uns die da jetət vielleicht die also da reinkommen würden en ganz, komischen Gefübl" (KPZ 03, Z. 56-59)

Es gab verschiedene Ursachen für die distanzierte Haltung des Personals gegenüber dem KPZ: Die Mitarbeiter befürchteten zum einen eine verstärkte eigene Konfrontation mit Sterben und Tod nach Eröffnung des Zimmers. Durch den Ausbau der ambulanten palliativmedizinischen Versorgung von Kindern und Jugendlichen, aber in geringem Umfang auch durch die Entstehung von Kinderhospizen, starben in den letzten Jahren weniger Kinder im Krankenhaus als vor diesem Ausbau der Versorgungsstrukturen. Zusätzlich verstarben statistisch gesehen im Krankenhaus die meisten Kinder auf der Intensiv- und weniger auf der Normalstation (vgl. Kapitel 3.4.4 Sterbeorte für Kinder). Einige Mitarbeiter befürchten nun, dass das KPZ das Sterben auf die Station ,zurückholen` würde, weil eine zusätzliche Versorgungsmöglichkeit geschaffen wurde:

„ja ich denke zum Beispiel dass man das Gefühl hatte das dann ständig da jemand drin liegt der wirklich halt (5) im Sterben liegt oder so"(KPZ 14, Z. 91-92)

Nach der Integration des KPZs in die Abteilung Neuropädiatrie war geplant, dass möglichst viele Gesundheits- und Kinderkrankenpfleger eine Pädiatrische Palliative Care Weiterbildung ${ }^{8}$ absolvieren sollten, um eine bestmögliche palliative Versorgung der Kinder im KPZ zu gewährleisten. Nach Beendigung des Vertrages mit KIMBU und der Entstehung des UMG-eigenen ambulanten Palliativdienstes auch im pflegerischen Bereich war geplant, dass auch Mitglieder des Pflegepersonals bei diesem ambulanten Dienst mitarbeiten (vgl. Kapitel 5.4.2 Aufbau UMG-eigener Versorgungsstrukturen für Palliativkinder). Da für die Sicherstellung eines 24-Stunden-Rufdienstes einige Mitarbeiter aus der Pflege benötigt werden und die meisten von ihnen wenig Bereitschaft zur Übernahme von Rufdiensten zeigten, wurde die Möglichkeit einer Weiterbildung in Palliative Care - in den Augen mancher Mitarbeiter - nun an die Bedingung der Teilnahme am ambulanten Kinderpalliativdienst geknüpft. Rufbereitschaft ist für das Pflegepersonal der Neuropädiatrie und Pädiatrischen Hämatologie und Onkologie unüblich und gehört nicht zum gewöhnlichen Aufgabenbereich. Diese empfundene Verknüpfung mit

\footnotetext{
8 Diese Weiterbildung kann z. B. an der Akademie Kinder-Hospiz Sternenbrücke in Hamburg absolviert werden. Sie wird nach dem Dattelner Curriculum gemäß festgelegter Qualitätsstandards veranstaltet. Die Weiterbildung umfasst vier Unterrichtsblöcke mit je 40 Unterrichtsstunden zu verschiedenen Themen und das Anfertigen einer Hausarbeit (Akademie Kinder-Hospiz Sternenbrücke)
} 
Bedingungen sorgte offenbar dafür, dass viele Mitarbeiter keine Weiterbildung in Palliative Care mehr machen wollten, weil besonders die Rufbereitschaft abschreckend wirkte: ,ja, das schreckt andere ab massiv also ich hab keinen gehört der fr-also ich hab von den Kolleginnen mit denen ich mich darüber unterhalten hab, is keiner bereit Rufbereitschaft zu machen" (KPZ 10, Z. 1014-1016)

Es stand außerdem im Raum, dass Kollegen, die bereits eine Weiterbildung gemacht hatten, arbeitsvertraglich zur Teilnahme am ambulanten Palliativdienst verpflichtet werden würden:

„,ne Kollegin hat ihn (Arbeitsvertrag) sich noch mal durchgelesen und da steht drunter man verpflichtet sich ₹u Mehrarbeit und ₹u Überstunden und ₹u Ruf- und eventuell ₹u Rufbereitschaften und ich denke das wird scheinbar ja jeder unterschrieben haben und die die jetzt die Fachweiterbildung schon haaben haben ja auch die fachlichen Voraussetzungen dafür diese Arbeit zu tun ich weiß nich (3) ich denke wahrscheinlich wird niemand geapungen werden einfach weil, das lässt ja dann die Motivation in den Keller wandern so irgendwie also das is ja kontraproduktiv aber ich glaube rein rechtlich glaub ich (2) spricht da nix dagegen demjenigen zu sagen bör mal zu wir baben dir die Weiterbildung bezaablt jetżt musst du balt auch Rufbereitschaft machen denk ich" (KPZ 10, Z. 1052-1062)

Für den geplanten Umbau des zuvor bestehenden Doppelzimmers, der den Patienten- und Familienbereich sowie das Badezimmer umfassten sollte, war die Grundfläche nicht ausreichend. In der Folge wurde ein Teil des angrenzenden Stationszimmers ${ }^{9}$ hinzugenommen. Obgleich diese Maßnahme kommuniziert und das Stationszimmer im Anschluss renoviert und hochwertig neu möbliert wurde, sorgte die Verkleinerung bei einigen Mitarbeitern für Unmut:

"und dazu kommt dass er jetzt deutlich kleiner is also wirklich deutlich kleiner" (KPZ 10, Z. 202-203)

Nach diesem Umbau kam es offenbar zu einem Stimmungsumschwung beim Personal: „, da kam viel Interesse und Neugierde und ach wie wird denn das ach ja was machen wir denn und so, son bisschen gekippt ist das glaub ich echt mit der Verkleinerung des Schwesternstütapunktes" (KPZ 7, Z. 849-851)

\footnotetext{
${ }_{9}$ Aufenthaltsraum der Gesundheits- und Kinderkrankenpfleger während der Pause und zur Schichtübergabe
} 
Diese Bedingungen führten dazu, dass das Personal das Thema KPZ bei Konfrontation zunächst abwehrte. Einige Mitarbeiter nahmen Abstand vom KPZ und seinen potentiellen Patienten, was in einer distanzierten Sprache („solchen Kindern“, „diesem Palliativzimmer") deutlich wurde:

„und bei solchen Kindern ist ja eben keine Verbesserung in Sicht (2)" (KPZ 02, Z. 632-633)

„hab ich da jetzt so meine ganz eigene Meinung zu diesem Palliativ₹immer" (KPZ 03, Z. 21)

Bedingt werden diese Handlungsstrategien auch dadurch, dass aufgrund eigener beruflicher Veränderungen das KPZ in den Hintergrund geriet:

„da hatte ich irgendwie grade erstmal damit zu tun mich auf der=diese Situation einzulassen die jetrt grade neu für mich war" (KPZ 02, Z. 88-90)

Aus der Ablehnung des KPZs resultierte ein dieses Thema betreffende Passivität und ein unsicherer Blick in die Zukunft. Zwar wird in den folgenden beiden Zitaten deutlich, dass die Interviewpartner davon ausgehen, dass es „sich einspielt“ und „Veränderungen ins Positive“ gehen, gleichzeitig scheint man unsicher zu sein, ob es wirklich passiert (,,ich glaub“) und wann genau „,es sich einspielt" (,irgendwann“, ,, dauert öfter länger“):

„ich glaub es wird sich irgendwann einspielen" (KPZ 17, Z. 464-465)

„aber mit Veränderungen die ins Postive gehn das dauert ja bekanntlicherweise öfter länger" (KPZ 01, Z. 496-497)

Den Handlungsstrategien liegen verschiedene intervenierende Bedingungen als generelle Vorbedingungen zu Grunde. Das heißt, es sind kontextuelle Faktoren, die bedingend und/oder verstärkend auf die Handlungsstrategie wirken können. Diese werden im Folgenden näher erläutert:

\subsection{Intervenierende Bedingungen der Kernkategorie}

Bedingt wurde die Ablehnung dadurch, dass scheinbar die baulichen Vorzüge sehr im Vordergrund standen, wodurch die inhaltlich-versorgungsbezogene ,Bedeutung ${ }^{6}$ des KPZs möglicherweise überdeckt wurde und eine Form der positiven Stigmatisierung entstand. Allgemein gesprochen, scheint eine Unterbringung in einem Palliativzimmer insoweit nicht erstrebenswert zu sein, wenn man sich vor Augen führt, dass eine Erkrankung in einem 
palliativen Stadium keine Aussicht auf Heilung mehr bietet. Das KPZ bietet jedoch so viele Vorteile, dass eine Unterbringung wünschenswert erscheint:

„wenn unsere Stammkunden die auch im Umgang nicht immer einfach sind einmal im Zimmer $9{ }^{10}$ warn und dann wieder kommen und wieder in ein anderes olles Zimmer müssen sagen / ((äfft eine Stimme nach)) wir waren aber in der 9 da warn wir immer und da kann man sogar auf den Balkon/ "(KPZ 07, Z. 1273-1276)

Zusätzlich fühlten sich die meisten Mitarbeiter vom Entstehungsprozess des KPZs ausgeschlossen, was ihre distanzierte Haltung begünstigte:

\subsubsection{Sich ausgeschlossen fühlen vom Entstehungsprozess des KPZ}

Es stellte sich heraus, dass sich viele Mitarbeiter über den Entstehungsprozess des KPZs nicht ausreichend informiert und in diesen auch nicht integriert fühlten. Offenbar folgte die Entscheidungsfindung einer klaren Hierarchie mit der Folge, dass die getroffenen Entscheidungen in der Wahrnehmung der Mitarbeiter von ihnen akzeptiert werden mussten. Diese hierarchische Entscheidungsfindung betrifft allgemeine Entschlüsse, wurde aber auch im Falle der KPZ-Entstehung deutlich. So berichteten viele Interviewpartner, dass der Beschluss ,dass` und ,wo' ein KPZ entsteht, den Mitarbeitern lediglich ,verkündet ${ }^{\star}$ wurde. Verdeutlicht werden kann dieses Gefühl des ,Ausgeschlossen-Seins“ im folgenden Zitat, welches sich auf die Bauphase des KPZ bezog. Während dieser Zeit fanden alle Arbeiten im abgesperrten und mit Planen verschlossenen Zimmer statt und der Bereich war dem Personal nicht zugänglich. Von außen betrachtet erscheint es nicht ungewöhnlich, dass ein Baustellenbereich unzugänglich ist, doch im folgenden Zitat wird deutlich, dass das Stattfinden von Handlungen im ,Verborgenen“ („da ist irgendwas"), wie es scheint, über das normale $\mathrm{Ma}$ an Baustellensperrung hinausging:

$$
\begin{aligned}
& \text { „also ja es wurde dann mit Planen zugeklebt und diese Planen da wurde dann ab und zu } \\
& \text { hat es gerattert oder so / ((belustigt)) mal nen bisschen Geräusche kamen dann/ und dann } \\
& \text { hat man gemerkt da ist irgendwas" (KPZ 15, Z. 38-41) }
\end{aligned}
$$

Es klang so, als wäre dieser Mitarbeiterin/diesem Mitarbeiter nicht klar, was genau dort hinter der Plane ,passiert'. So ist das Abdecken der Baustelle nicht nur auf diese selbst bezogen, sondern auch als Sinnbild für die ,Abdeckung' der mit dem KZP im Zusammenhang stehenden Planung und Vorgehensweise zu verstehen. Es kann angenommen werden, dass, wäre das Personal über die Abläufe des Entstehungsprozesses

\footnotetext{
${ }^{10}$ Das Kinderpalliativzimmer hat die Zimmernummer 9.
} 
informiert oder integriert worden, Kenntnis über die Vorgänge des Baustellenbereiches hätten vorhanden sein müssen und verbalisiert werden können. Das Gefühl des ,Ausgeschlossen-Seins` entstand bei den Mitarbeitern vor allem durch die wahrgenommene oder tatsächliche Intransparenz, von der der Entstehungsprozess begleitet wurde. So erhielten einige Mitarbeiter, nach eigenen Aussagen, nur wenige Informationen über Planung und Umsetzung. Viele hätten sich hier eine stärkere Integration in den Entstehungsprozess gewünscht:

„,bewuusst sind wir nich großartig gefragt wurden jedenfalls nich so die Mitarbeiter die hier arbeiten, kann ich mich nicht entsinnen man hat zwar immer mal etwas vorgestellt bekommen aber das man da noch mal so richtig gefragt worden ist was ist besser was ist schlechter kann ich mich nich bewusst entsinnen " (KPZ 18, Z. 121-125)

Doch auch nach Fertigstellung des Zimmers bestand viel Unklarheit des Personals bezüglich der Zimmerbelegung und zukünftigen Arbeitsanforderungen:

„das irgendwie kein klares Konzept oder dass es ja dass es so aussieht als gäbs keinvielleicht gibts ja eins aber das is irgendwie nich kommuniziert worden das klare Konzept keiner weiß so richtig, wie das jetzt laufen soll" (KPZ 10, Z. 77-80)

Das Personal wurde mit der Tatsache (Entstehung eines KPZs auf der eigenen Station) konfrontiert bzw. musste sich damit konfrontieren lassen. Der Informationsfluss fand hauptsächlich über Kollegen statt und schien wenig in Form offizieller Dienstankündigungen erfolgt zu sein. Auffallend war, dass einige Mitarbeiter durch Informationsplakate auf der Station und an verschiedenen Stellen in der Uniklinik von dem Zimmer erfuhren und nicht z. B. durch eine Informationsveranstaltung, was diese als unangemessene Informationsweitergabe empfanden:

„ich hab dadurch erfahren , durch das Plakat was bei uns auf-aushängt auf der Station und weil das natürlich unter den Schwestern oder dem Personal auf der Station diskutiert wurde, dadurch hab ich das erfabren, dass da nen Palliativitimmer eingerichtet werden soll" (KPZ 03, Z. 8-11)

Einige Mitarbeiter reagierten zunächst mit Distanzierung auf die Konfrontation mit dem Thema KPZ. Durch ihre tägliche Arbeit im bzw. mit dem KPZ sehen sie sich aber gezwungen, sich mit dem Thema KPZ auseinanderzusetzen und Position zu beziehen:

„und dann gingen da auch die Gespräche der Mitarbeiter los wie man dazu steht? , ob einem des gefällt? , dann hat man sich des erste Mal glaub ich so richtig damit auseinander gesetzt" (KPZ 02, Z. 17-19) 
Durch diese als Überrumpelung wahrgenommene Information und fehlende Einbeziehung reagierten viele der interviewten Mitarbeiter mit Kritik am KPZ. Die Kritik betraf vor allem subjektive bauliche und gestalterische Mängel des Zimmers, wie z. B. unpraktisches Mobiliar für die tägliche Versorgung des Patienten:

„müsste gleich direkt am Eingang ne Möglichkeit haben sich die Hände zu desinfiżeren , da is- war kein Platz für'n Händedesinfektionsmittel [...], dann muss man halt wenn der Patient (MRSA) oder was ähnliches hat müsste man balt auch äh Pflegekittel aufhängen können da gabs gar keinen Platr für [...], das is eine- dann ähm was war im Bad wars irgendwie der Wasserbahn, war so tief dass man überhaupt nich mit der Waschschüssel drunter kam oder so , [...] keine Sitzmöglichkeit da in dem in dem Bad irgendwie sonst äh zum Beispiel haben die Toiletten nen Klodeckel das hat diese Toilette da nich warum weiß ich auch nich [...] ne Ablagemöglichkeit im Bad war auch erst überbaupt gar nich da für irgendwelche Utensilien " $(K P Z$ 14, Z. 167-190)

Diese deutliche Kritik an der baulichen Gestaltung mag dadurch bedingt sein, dass bei manchen Mitarbeitern das Gefühl einer Scheinteilhabe am Entstehungsprozess aufkam. So wurde in manchen Dienstbesprechungen während der KPZ-Entstehung über das KPZ und den Fortgang bei den Bauarbeiten berichtet und das Personal auch nach seiner Meinung gefragt. Zugleich hatten einige Mitarbeiter aber den Eindruck, dass Anregungen des Personals nicht umgesetzt wurden:

„aber da würde man sich schon wünschen das man von vorneherein gesagt kriegt so das ist das Projekt da wolln wir hin und ähm aber gut." (KPZ 07, Z. 341-342)

Aus diesen Umständen resultierte, dass viele Mitarbeiter, die dem Projekt anfangs positiv gesinnt und sich auf das KPZ gefreut hatten, dem Zimmer anschließend skeptisch gegenüberstanden. Viele Rahmenstrukturen, die im Entstehungsprozess deutlich wurden und Veränderungen, die sich für das Personal ergaben, ließen Bedenken aufkommen:

„ich hab mich da eher so ein bisschen drauf gefreut, glaub ich also ₹u dem Zeitpunkt jedenfalls?" (KPZ 02, Z. 62-64)

Zugleich wird diese positive Äußerung jedoch rückblickend sprachlich relativiert, indem die Freude nur „ein bisschen“ vorhanden war und zudem angezweifelt wird („glaub ich“). Es zeigten sich außerdem intra- und interprofessionelle Konflikte zwischen den Mitarbeitern, welche die Zusammenarbeit erschwerten und die distanzierte Haltung zusätzlich begünstigten. 


\subsubsection{Intra- und interprofessionelle Konflikte belasten Zusammenarbeit}

$\mathrm{Zu}$ Beginn dieses Kapitels sei angeführt, dass in den Interviews häufig vom „Team“ (dt. Gruppe) gesprochen wurde. Aufgrund vielfältiger Definitionen und Verwendungen dieser Begrifflichkeit in der Literatur und der Alltagssprache wird in dieser Arbeit die Definition von Leuzinger und Luterbacher verwendet. Sie verstehen unter „einer Gruppe ein soziales System, dessen Mitglieder in direkter Interaktion stehen und in ihrem Verhalten durch komplementäre Rollen sowie gemeinsame Normen und Ziele bestimmt werden"(Leuzinger und Luterbacher 2000, S. 88). Daraus darf allerdings nicht geschlossen werden, dass es sich bei allen Mitarbeitern, die zur Erledigung von Aufgaben zusammengeschlossen werden, automatisch um Gruppen handelt. Die gemeinsame Arbeit hat nämlich nicht zwangsläufig zur Folge, dass ein „Wir-Gefühl“ entsteht und gemeinsame Normen und Ziele zur Bildung einer ,echten' Gruppe beitragen (Leuzinger und Luterbacher 2000). Dies war auch in der vorliegenden Studie zu beobachten. Nicht in allen beschriebenen „Teams“ gab es bei allen Interviewpartnern das „Wir-Gefühl“, sodass nicht alle Zusammenschlüsse von Mitarbeitern als „Gruppe“ oder „Team“ im Sinne der Definition bezeichnet werden können. Aus diesem teilweise fehlenden Zusammengehörigkeitsgefühl entstanden intraund interprofessionelle Konflikte. Diese Auseinandersetzungen zwischen den Mitarbeitern belasteten und erschwerten die Zusammenarbeit. Erschwert wurde die Zusammenarbeit auch durch Konflikte mit Vorgesetzten. Darunter zusammengefasst sind aktive Auseinandersetzungen im Sinne einer offen geäußerten Kritik an Handlungen und Entscheidungen, aber auch eine nicht offen geäußerte Kritik an z. B. organisatorischen Missständen. Es zeigte sich außerdem eine unklare und intransparente Teamkommunikation, sowohl innerhalb einzelner Professionen als auch inmitten des multiprofessionellen Teams:

$$
\begin{aligned}
& \text { „also so man kriegt das ja immer nur mit weil einem andern Leute das erzäblen also da } \\
& \text { gibts ja nie so ne Teambesprechung wo gesagt wird so und so ist das jetzt wo man wirklich } \\
& \text { mal so offizielle Informationen hat" (KPZ 02, Z. 217-219) }
\end{aligned}
$$

Dies wurde vor allem dadurch deutlich, dass offenbar sehr viel Informationsaustausch durch Hörensagen und über Dritte stattfand und wenig durch direkte Kommunikation.

Es zeigten sich außerdem Uneinigkeiten zwischen den Professionen Pflege und Ärzteschaft bezüglich Therapieentscheidungen bei Patienten. Ärzteschaft und Pflege nahmen - aus Sicht des Pflegepersonals - Patientensituationen teils unterschiedlich wahr. Dies betraf vor allem die Schmerzempfindung von Patienten. Viele Angehörige des Pflegepersonals waren der Meinung, dass seitens der Ärzte Schmerzen von Patienten unterschätzt und nicht 
ausreichend medikamentös therapiert wurden. Inwieweit dies objektiv der Fall ist, blieb unklar und kann an dieser Stelle nicht überprüft werden. Diese Konflikte wurden teils offen ausgeführt, teilweise wurde diese Kritik nur im Interview bzw. gegenüber Kollegen geäußert:

„und dann gibt es auch manchmal Diskrepanzen zwischen Äraten und Schwestern was man jetzt da macht und was man nicht macht und hat der jetzt Schmerzen und wieviel soll der jetzt haaben so" (KPZ 14, Z. 510-513)

Diese Vorsicht bei der Schmerzmitteldosierung schien - nach Meinung des Pflegepersonals - auch dadurch bedingt zu sein, dass manche Ärzte juristische Konsequenzen verzweifelter Eltern befürchteten, falls höhere Schmerzmitteldosen das Leben des Kindes verkürzen würden:

,und sind immer noch der Meinung sie werden sofort verklagt wenn sie irgendwie mal 2 Milligramm Morphium geben" (KPZ 07, Z. 943-944)

Viele Mitarbeiter distanzierten sich deshalb im Interview von Handlungsweisen und Ansichten ihrer Kollegen. Dies betraf Verhaltensweisen und Einstellungen im Allgemeinen, aber auch speziell auf das KPZ bezogen. So war auffällig, dass z. B. wenige Mitarbeiter offen über ihre persönlichen Bedenken bezüglich des KPZs sprachen, aber nahezu alle von Bedenken ihrer Kollegen berichteten:

„es sind viele Ängste mit dabei sowobl bei Äraten als auch in der Pflege" (KPZ 17, Z. 322-323)

„ich finds nich so schlimm aber ich weiß dass sich Kolleginnen sebr aufregen" (KPZ 10, Z. 211-213)

Um dem interprofessionellen Konflikt bei Therapieentscheidungen entgegenzuwirken, wurde auch vorgeschlagen, objektive Beurteilungsverfahren, wie z. B. Schmerzskalen einzuführen, um die Entscheidungen zu objektivieren. Einige Mitarbeiter schätzten es besonders, wenn Vorgesetzte Verantwortung übernahmen und dadurch Ruhe und Sicherheit vermittelten, aber auch, wenn sie sich für die Interessen ihrer Mitarbeiter aktiv einsetzten. Es kam allerdings vor, dass sich Mitarbeiter selbst aus dem Team ausschlossen, weil ihnen vielleicht die Umgangsform missfiel oder sie sich nicht ausreichend integriert fühlten. Dies wurde nicht alles unmittelbar durch das KPZ bedingt, sondern manche Konflikte bestanden schon zuvor:

„also so is es nich ne, aber es is schon so nen bisschen Einzelkämpfer is man dann schoon" (KPZ 03, Z. 648-649) 
Generelle Vorbedingungen für Handlungsstrategien des Personals war die Vermutung besonders aus den Reihen des Pflegepersonals - dass die Ärzte stärker einer tatsächlichen oder vermeintlichen Hierarchie unterliegen, von der sie abhängig sind. Es wurde zudem vermutet, dass sie sich deshalb weniger für Stationsinteressen einsetzten und kaum öffentlich kritisierten, weil sie meist nicht allzu lange Zeit auf einer Station bleiben. Angehörige des Pflegepersonals arbeiten teils über Jahrzehnte auf einer Station und kämpfen deshalb wohl viel intensiver gegen Veränderungen am Arbeitsplatz, die ihnen missfallen:

\section{„die Kulturen sind da anders im äratlichen Bereich ist ne ganz, andere Betriebskultur irgendwie Assistenzärz̧te oder auch Oberärzte die Hierarchie ist da viel ausgeprägter und die wissen was der Chef sagt das wird gemacht und da wird ja auch viel weniger gekämpft weil die alle Karriere machen wollen" (KPZ 07, Z. 352-356)}

Als Konsequenz aus den inter- und intraprofessionellen Konflikten wurde deutlich, dass sich einige Mitarbeiter ausgeschlossen fühlten bzw. vom Team ausgeschlossen wurden. Dies zeigte sich z. B. darin, dass eine Verunsicherung entstand, wenn man mit seinen Sorgen und Ängsten scheinbar alleine dastand, weil die Bedenken von den Kollegen nicht geteilt wurden. Doch entwickelte sich das Gefühl des ,Ausgeschlossen-Seins“ auch, wenn man nicht dabei war, wenn scheinbar wichtige Informationen verkündet wurden. Und auch Mitarbeiter, die ihre Bedenken bzw. Kritik am KPZ offen äußerten, fühlten sich danach häufig vom Team ausgeschlossen, weil viele sich von offen geäußerten Ängsten und Bedenken distanzierten:

„ich war schon überrascht auch das angeblich so viele Koll=Kollegen doch das positiv auch fanden " (KPZ 01, Z. 25-26)

Neben dieser, durch strukturelle und organisatorische Schwierigkeiten verursachten distanzierten Haltung gegenüber des KPZs zeigten sich auch emotionale Gründe, die den Implementierungsprozess belasteten. Die Betreuung und Arbeit mit schwerstkranken und sterbenden Patienten stellt für das Personal insgesamt eine große Herausforderung dar.

\subsubsection{Sterben als professionelle Herausforderung}

In den Interviews kam zum Ausdruck, dass die Konfrontation mit sterbenden Patienten für Mitarbeiter eine große emotionale Belastung und eine Herausforderung im Arbeitsalltag darstellen kann. Ersichtlich wurde dieses Phänomen an folgender Stelle, an der eine der interviewten Personen sich fragte, ob sie/er ein „Todesengel“ sei, nachdem wiederholt Kinder in der eigenen Schicht verstorben waren: 
„zu Beginn meines Berufslebens da hab ich mich echt gefragt ob ich ein Todesengel" bin [...] eigentlich immer wenn da Kinder gestorben sind wars da immer eigentlich auch in meinen Diensten also wenige Ausnabmen wirklich ganz wenige [...] ich bab mich auch gefragt mach ich irgendwie was was die instabiler macht" (KPZ 07, Z. 422429 und 446-447)

An dieser Textstelle wird deutlich, wie belastend der Tod eines Patienten sein kann und dass Selbstzweifel durch wiederholte Todesfälle ausgelöst werden können. Dies zeigt sich besonders bei der Bezeichnung als „Todesengel“ (vgl. Fußnote 11 Seite 65). Es steht außer Frage, dass die Interviewpartnerin bzw. der Interviewpartner nicht den Tod eigener Patienten herbeigeführt hat. Die Betitelung als „Todesengel“ kann vielmehr als Ausdruck dafür verstanden werden, wie beängstigend eine gefühlte Häufung von Todesfällen während der eigenen Dienste wirken kann. Diese Angst kann Zweifel am eigenen Handeln aufkommen lassen („mach ich irgendwie was was die instabiler macht") und für Verunsicherung sorgen. Diese professionelle Herausforderung steht im Kontext einer allgemeinen Schwierigkeit den Tod ins Leben zu integrieren. Damit ist gemeint, dass manche Mitarbeiter versuchten, den Tod aus der alltäglichen Vorstellung auszublenden. Er wird als etwas ,Furchtbares' wahrgenommen, worüber nur nachgedacht wird, wenn dies unbedingt erforderlich ist. Des Weiteren zeigte sich, dass der Sterbeort eines Kindes einen besonderen Einfluss auf die Trauerbewältigung des Personals hat. So empfanden es manche Mitarbeiter als weniger belastend, wenn ein Kind Zuhause verstarb und sie nur über den Tod informiert wurden, als wenn sie unmittelbar und direkt in das Sterben involviert waren. Solche Empfindungen können eine Begründung für die ablehnende Haltung von Mitarbeitern gegenüber dem KPZ sein:

\section{,für mich für meine Tätigkeit eine Entlastung wenn die Kinder Zubause versterben können ja weil ich nicht unmittelbar damit konfrontiert bin nicht unmittelbar sehend ja tätig werdend sondern mit Abstand über Telefonaate ja" (KPZ 08, Z. 270-} 273)

\footnotetext{
${ }^{11}$ Der Begriff „Engel“ beschrieb ursprünglich einen „Boten zwischen Gott und der Menschenwelt“. Im Verlauf der Geschichte wurde er in den monotheistischen Religionen zu einem „Gattungsbegriff für alle Arten von Geisterwesen“ (Krauss 2002, S. 7). Ein Engel, der den Menschen den Tod bringt (=Todesengel), wird in der Bibel nicht erwähnt. Unter dem Einfluss der griechischen Philosophie entwickelt sich im Christentum aber die Vorstellung eines Engels, der die Seele des Menschen von seinem Leib trennt. Im Koran wird ein Engel des Todes - Azrail - ausdrücklich erwähnt (Krauss 2002). In der Journalistik findet die Bezeichnung „Todesengel“ z. T. auch Verwendung für medizinisches Personal, welches den Tod eigener Patienten herbeigeführt hat.
} 
Anderen Interviewpartnern fiel es jedoch deutlich leichter, den Tod eines Patienten zu verarbeiten, wenn sie direkt mit dem Tod konfrontiert waren:

„manchmal kommt dann nur son Anruf ja die ist gestorben das ist manchmal viel schlimmer also man das ist dann so abstrakt und man weiß gar nicht wohin mit seiner Trauer und wenn man selbst da aktiv beteiligt war also mir gehts jedenfalls so ich kann das dann besser verarbeiten" (KPZ 07, Z. 438-441)

Es gab jedoch auch Mitarbeiter, für die der Todesort eines Kindes und die Frage ihrer Anwesenheit während des Sterbens keinen Einfluss auf die persönliche Trauerbewältigung hatten. Es wird somit deutlich, dass der Einfluss des Sterbeortes auf die Trauerbewältigung von den Mitarbeitern sehr unterschiedlich empfunden wird.

Das Sterben eines Patienten bzw. die Sterbebegleitung stellte häufig eine psychische Belastung für die beteiligten Mitarbeiter dar. Diese Belastung war ursächlich für die professionelle Herausforderung, die das Sterben eines Patienten bedeutete. Es entstand ein Gefühl der Macht- und Hilflosigkeit gegenüber den Patienten und Eltern, zu denen im (meist) langen Krankheitsverlauf eine zum Teil enge Beziehung entwickelt wurde und die man trotz allem nicht vor dem Tod bewahren konnte:

„aber dass es schon was ist was auch an die Substanz,geht 'bei allen'“(KPZ 03, Z. 94)

Es entstanden teilweise Selbstzweifel, dass vielleicht nicht alles getan wurde, um den Patienten zu vor dem Tod zu bewahren. Jeder Mitarbeiter musste Strategien und Wege finden, um diese psychische Belastung zu bewältigen. Gleichzeitig verdeutlichten einige Mitarbeiter ihre Angst vor der Präsenz von Tod und Sterben, welche das KPZ hervorrief. Es wirkte wie eine Angst vor einem diffusen Gegner. Es ging hierbei weniger darum, dass das Sterben eines Patienten emotional belastend war, sondern eher um eine Angst vor dem Sterben und dem Tod an sich. Allein die Möglichkeit, dass im KPZ Kinder versterben könnten, löste Beklommenheit aus:

„ja und dass man so diese Angst äh vor dem direkten Sterben “ (KPZ 18, Z. 692-693)

Für einzelne Mitarbeiter ging vom KPZ eine so starke Bedrohlichkeit und Störung der beruflichen Zufriedenheit aus, dass ein Arbeitsplatzwechsel in Erwägung gezogen wurde:

„das Pallizimmer das ist für mich schon ne Hürde ich also, wenn mich das zu sebr belastet wär das für mich ein Grund die Station zu verlassen" (KPZ 01, Z. 338-339) 
Das Personal hatte verschiedene Strategien entwickelt, um mit dieser Herausforderung umzugehen, indem sie z. B. auf ein kollegiales Netz zurückgriffen und fachliche und emotionale Unterstützung bei Kollegen fanden:

$$
\begin{aligned}
& \text { „im Team spricht man natürlich dann schon auch mal einfach im Pausenraum drüber, } \\
& \text { wie gehts dir und so, es trifft einen ja mal mehr oder weniger dann persönlich aber man } \\
& \text { geht ja schon in Kontakt auch" (KPZ 04, Z. 374-376) }
\end{aligned}
$$

Es wurde aber auch versucht, Abstand zu gewinnen, indem z. B. weniger schwer erkrankte Kinder versorgt oder sich Freiräume z. B. durch Stundenreduzierung geschaffen wurden:

$$
\begin{aligned}
& \text { „ja dass man einfach nen bisschen Abstand gewinnt dass man dann einfach mal wieder } \\
& \text { normale Patienten betreut wo wirklich nur Diagnostik is wo, der normale Ablauf läuft } \\
& \text { so obne dass man tiefschürfend sich reindenken muss" (KPZ 18, Z. 737-740) }
\end{aligned}
$$

In diesem Zitat ist die Bezeichnung „normale Patienten“ als Definition für nicht-sterbende Kinder und somit die Beschreibung von sterbenden Kindern als ,unnormale Patienten“ auffallend. Dies verdeutlicht wieder die emotionale Belastung, die die Versorgung sterbender Kinder bedeutet. Das Personal versuchte auch einen versöhnlichen Abschluss mit dem Tod eines Patienten zu finden. Zum einen emotional, indem eigene Rituale geschaffen wurden, die es ermöglichten, mit dem Tod eines Patienten abzuschließen. Zum anderen eher kognitiv, indem der Tod eines Kindes nach langer und schwerer Krankheit als „Erlösung“ definiert wurde:

$$
\begin{aligned}
& \text { „also ich glaub dass die Verarbeitung von nem Patient der stirbt, in ner } \\
& \text { Palliativsituation (2) oft oder fast immer als Erlösung wahrgenommen wird" (KPZ 11, } \\
& \text { Z. 426-427) }
\end{aligned}
$$

Eine fehlende Distanz zwischen Mitarbeitern und Patienten und Angehörigen konnte wahrgenommen werden, wenn sich Mitarbeiter über den beruflichen Kontext hinaus engagierten, z. B. indem sie einen persönlichen Kontakt zu den Eltern eines verstorbenen Kindes aufrechterhielten oder einem Kind und seiner Familie Geschenke machten. Diese Strategien des Personals, mit dem Tod als professionelle Herausforderung umzugehen, wurden durch verschiedene Vorbedingungen beeinflusst: Sterbende Kinder stellen heute aufgrund des medizinischen Fortschritts - eine gefühlte Umkehr der ,natürlichen Ordnung dar und sind aus der Perspektive einer interviewten Person auch gesellschaftlich nur schwer akzeptiert. Dies erschwert die emotionale Bewältigung der eigenen Arbeit zusätzlich: 
„ich mein (4) Sterben is sowieso immer $n$ Tabuthema und wenn $n$ Kind stirbt wenn $n$ Jugendlicher stirbt is das ja für die meisten Leute kaum auszuhalten" (KPZ 11, Z. 659661)

Daneben ist die kurative Zielsetzung eines Krankenhauses mit Ausschöpfung aller medizinischer Optionen offenbar stark präsent:

„,ich glaub bier im Krankenhaus füblt man sich auch immer so in der Verpflichtung dem Patienten noch irgendwie belfen zu müssen" (KPZ 16, Z. 432433)

Die Mitarbeiter haben, wie es scheint, auch den Eindruck, dass ein gesellschaftlicher Anspruch an ihr Handeln besteht, mit der Forderung eines professionell-distanzierten Verhaltens. Der Tod eines Menschen darf berühren, jedoch nur in einem solchen Maß, dass die Arbeit trotzdem noch professionell ausgeübt werden kann. Allerdings konnten auch traumatische Vorerfahrungen, wie z. B. ein sehr belastender erster Kontakt mit einem sterbenden Kind oder die unzureichende Vorbereitung auf Sterbebegleitung in Ausbildung und Studium die Handlungsstrategien beeinflussen. Einzelne Interviewpartner hatten den Eindruck, dass das Geschlecht den Umgang mit psychisch belastenden Situationen beeinflusst:

„es gibt ja unterschiedliche Verarbeitungs- formen erfahrungsgemäß sprechen Frauen hilft es ibnen mebr darüber zu sprechen und Männer machen Sachen mehr mit sich ab" (KPZ 04, Z. 274-276)

Dieser verschiedenartige Umgang wurde zum einen bei Kollegen wahrgenommen (professionsunabhängig) und zum anderen auch bei betroffenen Vätern und Müttern. Dies bedingte deren Umgang mit psychisch fordernden Gegebenheiten und konnte zusätzlich $\mathrm{zu}$ Kommunikationsschwierigkeiten und Unstimmigkeiten im Team und zwischen Mitarbeitern und Eltern führen.

Des Weiteren konnte es durch die Arbeit mit schwerstkranken Kindern zu einer veränderten Sicht auf Krankheit und Gesundheit im Privaten bei den Mitarbeitern kommen. Die Analyse ergab, dass berufliche Erfahrungen mit ins häusliche Umfeld genommen wurden, beispielsweise konnte der tägliche Umgang mit erkrankten Kindern dafür sorgen, dass die Angst entstand, auch die eigenen Kinder könnten schwer erkranken:

„wenn sie dann anfangen Ihr eigenes Kind zehnmal am Tag zu überprüfen ob die blauen Flecken irgendwie Lenkämie sein können oder nich wenn man als Kind grade vom Spielplatz kommt dann wirds schwierig" (KPZ 08, Z. 570-573) 
Die veränderte Sicht auf Krankheit und Gesundheit konnte sich aber auch positiv äußern, indem man die eigene Gesundheit oder die der eigenen Kinder viel mehr zu schätzen wusste, wenn einem bekannt war, an welchen Krankheiten Kinder leiden und versterben könnten. Die veränderte Wahrnehmung von Krankheit und Gesundheit wurde nicht durch das KPZ selbst, sondern durch die Arbeit mit schwerstkranken Kindern auf der Station an sich beeinflusst. Zusätzlich schien diese Arbeit einen Einfluss auf den Umgang mit der eigenen Spiritualität und Sterblichkeit zu haben:

„und das es mir wenns bei mir mal soweit ist helfen wird da bin ich ganz sicher" (KPZ 7, Z. 635-636)

Es existierten einvernehmliche Rituale der Sterbesituation unter den Mitarbeitern der Stationen, wie z. B. der Stationsalltag in einer Sterbesituation organisiert wurde (Aufgabenverteilung, Betreuungsschlüssel, Kontaktpflege nach Tod des Kindes). Dies konnte dem einzelnen Mitarbeiter Sicherheit und Halt in der belastenden Situation geben. Es gab allerdings Mitarbeiter, die dieser psychischen Belastung der Sterbebegleitung nicht dauerhaft gewachsen waren und das Arbeitsfeld wechselten. Dies fand nicht im Zusammenhang mit der KPZ-Entstehung statt, sondern wurde als Erfahrung aus der Vergangenheit berichtet. Dies verdeutlicht jedoch die mit dem Sterben verbundene Anspannung, die durch das KZP noch einmal stärker an Präsenz gewinnen und die Belastung erhöhen könnte.

Einige Mitarbeiter hingegen schätzten die Arbeit mit sterbenskranken Kindern und die Sterbebegleitung selbst, weil sie es für eine wertvolle Arbeit hielten für die sie, wenn sie gut erledigt würde, auch Wertschätzung z. B. von den Eltern erhielten:

„zum Teil man kriegt en ja typischerweise so wenn der Todeszeitpunkt eines Kindes sich nähert jährt so kriegt man dann ab und zu nochmal en Brief von Eltern also nicht oft aber gelegentlich war das wo dann drinstand wie gut das gelaufen ist und wie froh sie warn das man da war und (2) das bestärkt einen natürlich das gibt unbeimlich viel fürs Ego" (KPZ 07, Z. 472477)

\subsection{Konsequenzen der Kernkategorie}

Die Handlungsstrategien hatten verschiedene Konsequenzen zur Folge: Zum einen wurde eine andere medizinische Versorgung und Genesung bei Unterbringung im KPZ antizipiert. Einige Mitarbeiter hatten das Gefühl, dass sich die Versorgung durch das KPZ 
insofern verbesserte, als dass sie sich auch als Behandler dort lieber aufhalten und der Patientenversorgung mehr Zeit widmen würden:

„is jetat ne reine Phantasie von mir aber wenn ich da reinkomme und und fübre ein Gespräch nebm ich mir vielleicht auch inst- unbewusst nich bewusst aber unbewusst en bisschen mehr Zeit weil ich den Ort einfach angenehm finde da halte ich mich einfach gerne auf, ja wenn, in nem Ort in nem Zimmer der jetzt was eng ist wo ich sach ma ,n schreiendes Kind nebendran is sowieso nich da hab ich ob Gott jetzt muss ich hier schnell wieder raus damit ich irgendwie nen klaren Gedanken fassen kann und so also das sind so kleine Faktoren die ne Rolle spielen" (KPZ 15, Z. 381-388)

Aber auch Therapiemöglichkeiten schienen durch das ausgedehntere Platzangebot und die moderne Ausstattung vergrößert zu sein. Obgleich für alle Eltern Raum und Zeit für Gespräche zur Verfügung steht, wurde antizipiert, dass dies im Aufenthaltsbereich des KPZs für die betroffenen Eltern problemloser ist, da Raum und Ruhe gegeben ist und das Kind trotzdem nicht allein gelassen werden muss. Nach Einschätzung des Personals wollten Eltern ihre Kinder während solcher Gespräche nur ungern im Zimmer allein lassen, was bei einem Gespräch im Arztzimmer notwendig wäre. Es wurde auch die Hypothese aufgestellt, dass sich die Genesung bzw. der Gesundheitszustand bei Versorgung im KPZ stabilisieren oder sogar verbessern würde. Dies wurde zurückgeführt auf die angenehme und privatere Atmosphäre im KPZ, sowie die neue und behindertengerechte Ausstattung, die die Arbeit des Personals zu erleichtern und das Versorgungsniveau scheinbar steigen ließ:

„in den Gesprächen die man dort fübrt, mehr Hoffnung geben kann kann sagen okay das äh ist sieht doch alles nich so schlimm aus es gibt doch ne Perspektiive und es geht weiter ja also ich glaube einfach in sonem Raum lassen sich diese Sätze leichter formulieren als in einem Raum der eng ist wo man das Gefühl hat ja eigentlich möchte ich hier so schnell wie möglich raus ja? das ist beengt es ist laut es ist nich komfortabel" (KPZ 15, Z. 346-351)

Bei all den Vorzügen stellte sich für das Personal die Frage, ob aus der „Klassengesellschaft“ auf der Station eine ,Zwei-Klassen-Medizin“ resultierten könnte. Folgende Aussage brachte die Frage nach einem menschenwürdigen Tod als zusätzliche Konsequenz ins Spiel:

„ich glaube eher es is auch'n Zimmer da wo man menschenwürdig sterben kann" (KPZ

15, Z. 649-650) 
Dies warf die Frage auf, was genau ein „menschenwürdiger“"12 Tod ist und ob er Kindern in anderen Zimmern verwehrt bleibt. Einige Mitarbeiter hatten zudem die Vision einer geteilten Station. Sie stellen sich vor, dass eine Kinderpalliativstation entstehen würde, die genau wie die Palliativstation für Erwachsene, eine eigene, abgetrennte Station und nicht in eine Normalstation integriert ist. Sie sahen darin die Möglichkeit, die gefühlte Entstehung einer „Zwei-Klassen-Station“ zu verhindern:

„dass das dann der Palliativbereich ist und es gibt natürlich den Punkt an dem man das dann abgrenzen muss das man das die Eltern oder das die Kinder und Eltern auf Station nicht das Gefühl haben das ist ne Zwei Klassen Station bier" (KPZ 07, Z. 1267-1270)

Die Distanzierung des Personals vom KPZ hatte eine Resignation zur Folge, welche bei einigen Personen zu Überforderung und Selbstunsicherheit führte.

\subsection{1 Überforderung und Selbstunsicherheit}

Die Analyse hat gezeigt, dass unter den Mitarbeitern ein unsicheres Wissen über die Aufgaben und Ziele von Palliativmedizin besteht. Für viele der interviewten Mitarbeiter war „palliativ“ gleichgesetzt mit Sterben, was in dem folgenden Zitat sehr gut zum Ausdruck kommt:

$$
\begin{aligned}
& \text { „ich muss mich noch von diesem Gedanken verabschieden für mich ist Palliativzimmer } \\
& \text { und Palliativkind ist halt wirklich das Kind was jetzt akut lebensbedrohlich ist ja also } \\
& \text { ähm und nicht mein Kind wo ich weiß ok das wird } 14 \text { Jahre alt nicht werden ja" (KPZ } \\
& \text { 01, Z. 375-378) }
\end{aligned}
$$

Diese Begriffsunsicherheit stellte ein großes Problem für die Arbeit der Mitarbeiter im und mit dem KPZ und den dort behandelten Kindern dar. Bei dieser Begriffsunsicherheit zeigte sich auch, dass Patientengruppen von einzelnen Mitarbeitern fast schon stigmatisiert wurden. Dies wird an den verwendeten Begrifflichkeiten („sabbern“, „,verkrüppelt“) im nachfolgenden Zitat deutlich. Diese abwertende Sprache betraf besonders

12 Artikel 1 des Grundgesetztes besagt, dass die Würde des Menschen unantastbar ist. Doch was genau ist nun menschenwürdiges Sterben? Mit dieser Frage haben sich Jens und Küng (1995) beschäftigt. Für sie ist z. B. der Tod eines Menschen durch Krieg, Hunger oder Epidemien ein menschenunwürdiger Tod. Ebenso wie das Fehlen sozialer und zwischenmenschlicher Kontakte, also ein Sterben in Einsamkeit, ohne körperlichen Kontakt und seelische Geborgenheit. Einsamkeit entsteht ihrer Meinung nach auch, wenn die Kommunikation zwischen Patient und Behandlern und Angehörigen beendet wird und mit dem Patienten nicht offen über seine Krankheit und das Sterben geredet wird. Sie führen an, dass auch ein Sterben in einem schönen Einzelzimmer nicht menschenwürdig ist, wenn Fürsorge durch Personal und Angehörige fehlen. Bedenkt man all dies so wird klar, dass das KPZ allein das Sterben nicht menschenwürdig macht. Eine liebevolle Betreuung des Patienten von Personal und Angehörigen und eine offene Kommunikation, die den Patienten mit seinen Ängsten nicht alleine lässt, kann im KPZ stattfinden, doch ist das KPZ keine Bedingung dafür. 
schwerstbehinderte Kinder, welche auf der Neuropädiatrie häufig behandelt werden und im Sinne der Einteilung der Erkrankungen nach der ACT (vgl. Kapitel 2 Einleitung) eine palliative Versorgung benötigen. Bei ihnen handelte es sich folglich häufig um Palliativpatienten (im Sinne der Definition), welche vom Zimmer profitieren sollten. Werden behinderte Patienten auch nur von einzelnen Mitarbeitern sehr distanziert behandelt, lässt dies dennoch den Schluss zu, dass eine umfassende Aufklärung zur Definition und Details von Palliativmedizin dringend erforderlich ist:

„auch so der Umgang mit Schwerstbehinderten die ja auch nicht immer nett freundlich, lieblich sind gehört ja auch daحu viele Kinder sabbern viele Kinder haben Krampfanfälle, ja sind ver-verkrüppelt hört sich so doof an aber mir fällt grad kein anderes Wort ein ne also da gehört auch was daحu" (KPZ 18, Z. 572-575)

Im Laufe der Interviews wurde ferner deutlich, dass eine Veränderung des Patientenklientels durch das KPZ nicht unmittelbar erwartet wurde. Schon vor der Erweiterung der Versorgungsstrukturen für Palliativkinder an der UMG waren - nach Einteilung der ACT (vgl. Kapitel 2 Einleitung) - Kinder, die eine palliativmedizinische Versorgung benötigen, Patienten in dieser Klinik. Dies schien zwar einigen, aber nicht allen Mitarbeitern bewusst zu sein:

„,da ich glaube das die , 'Station' sowieso sebr häufig Palliativkinder hat und jetżt gibt man einem Zimmer einfach nur noch nen anderen Naamen dafür und, hat andere Rahmenmöglichkeiten die man diesen Kindern und der Familie dafür bieten kann, weil das Klienteel glaub ich ändert sich dadurch nicht? (KPZ 02, Z. 57-62)

An dieser fragenden und unsicheren Art in diesem Zitat zeigte sich jedoch, dass einige Mitarbeiter befürchten, zukünftig doch mit anderen Patienten konfrontiert zu sein. Diese Vorstellung schien manche Mitarbeiter zu verunsichern.

Ursächlich für die auftretende Überforderung und Selbstunsicherheit war zunächst die für die Mitarbeiter der Neuropädiatrie neu hinzukommende Auseinandersetzung mit onkologischen Krankheitsbildern. Da das KPZ auch Patienten der pädiatrischen Hämatologie und Onkologie zur Verfügung steht, hatten viele Mitarbeiter der Neuropädiatrie Angst mit - ihnen nicht vertrauten - Krankheitsbildern und neuen fachlichen Anforderungen konfrontiert zu werden. Dieser hinzukommenden fachlichen Anforderung fühlten sich nicht alle Mitarbeiter gewachsen: 
„ich hab auch nich genug onkologische äh Erfahrung auch auch Wissen um das jetrt dem Patienten wirklich grad für spezielle Fragestellungen richtig zu betrenen" (KPZ 15, Z. $555-557)$

Daneben herrschte aber auch eine Unklarheit über das Versorgungskonzept des KPZs und die inhaltlichen Arbeitsanforderungen, die vermutlich an das Personal gestellt werden würden. Dies betraf vor allem die Frage nach der Zimmerbelegung und den neuen Erwartungen des Arbeitgebers, die als Fortsetzung der schon während der KPZEntstehung wahrgenommenen Intransparenz (vgl. Kapitel 5.5.1 KPZ als organisatorische Herausforderung) angesehen wurde. Nach Beendigung der Bauarbeiten und Eröffnung des Zimmers waren vielen Mitarbeitern die inhaltlichen Arbeitsanforderungen noch immer nicht genau genug definiert, sie mussten jedoch mit der Arbeit beginnen. Sorgte die Unklarheit während der Bauphase noch für Unmut, so entstand nach Zimmereröffnung Überforderung, weil der Arbeitsauftrag nicht eindeutig erschien:

„ich finde das nich so nen richtiges Konzept gibt oder jedenfalls dass es nich so bekaannt is was jetzt- es gibt keine klaren Regeln sagen wir mal so ähm keiner weiß so richtig wie das jetzt funktionieren soll oder $w$ - ja welche Patienten da jetz̨t rein sollen ob das jetz̨t auch mit normalen Patienten belegt werden soll obs freigehalten werden soll" (KPZ 10, Z. 45-49)

Viele Mitarbeiter fühlten sich zudem fachlich nicht ausreichend ausgebildet und vorbereitet für die Arbeit im KPZ und sahen besonders dort noch Nachbesserungsbedarf: „und das hätte ich mir gewünscht dass das besser vorbereitet wird dass die die Ängste genommen werden dass eben da schon mal, einfach ne Fortbildung stattfindet zu diesem Thema und zwar, weit vorher schon dass einfach die Ängste genommen werden " (KPZ 10, Z. 296-299)

Zahlreiche Mitarbeiter sahen deshalb in einer fachlichen Weiterbildung (Pädiatrische Palliative Care Weiterbildung oder klinikinterne Fortbildungen zum Thema Sterbebegleitung u. ä.) die nahezu einzige Möglichkeit, der Überforderung entgegenzuwirken:

,ja und das kann man halt find ich auch nur erreichen durch Fortbildungen deswegen also die müssen auf jeden Fall auch noch stattfinden aber da würd ich auch dran bleiben also da würd ich auch echt äh sonst ((lacht)) also da würd ich mich auch gegen wehrn also das muss auch sein. das wir da wenigstens Fortbildungen bekommen." (KPZ 01, Z. 181 185) 
Wichtig erschienen einigen Mitarbeitern auch regelmäßige Weiterbildungsangebote, um eine Überforderung und Unsicherheit dauerhaft zu verhindern:

„von einer Fortbildung kann maan nicht profitieren da muss man wirklich am Ball bleiben und mehrere und regelmäßig an Fortbildungen teilnehmen" (KPZ 01, Z. 618-

Während manche Mitarbeiter die Bringschuld beim Arbeitgeber sahen, versuchten sich einzelne auch eigenständig fachlich weiterzubilden:

„ich werd vielleicht noch Fortbildungsangebote nutzen oder zumindest mich in der Literatur so'n bisschen da noch informieren "(KPZ 03, Z. 559-561)

Trotz der subjektiven Überforderung wird hier deutlich, dass augenscheinlich ein „bisschen“ Information ausreichen würde, um auf das neue Tätigkeitsfeld vorbereitet zu sein. Dies zeigt einmal mehr, wie wenig Kenntnis in diesem Versorgungsbereich offenbar bereits besteht. Einige versuchten allerdings auch, durch eine aktive Auseinandersetzung mit dem Thema KPZ und Palliativmedizin im Allgemeinen ihrer Überforderung und Unsicherheit entgegen zu wirken. Sie setzten sich bewusst mit dem Zimmer auseinander, z. B. indem sie Ausklärungsarbeit bei Eltern, deren Kinder auf der Station untergebracht waren oder deren Kinder in das KPZ verlegt werden sollten, leisteten. Einzelne Mitarbeiter begannen auch, Zukunftsvisionen für den Kinderpalliativbereich in Göttingen zu entwickeln. So überlegten sie beispielsweise, ob in den nächsten Jahren der Bereich immer stärker erweitert werden und mehr Kollegen eine Weiterbildung in Palliativ Care haben würden.

In den Erzählungen der Interviews war auffällig, dass einige Mitarbeiter Euphemismen verwendeten, als sie über das KPZ sprachen. So wurden Begriffe wie „Hotelzimmer“ oder „Luxussuite“ verwendet, welche allesamt sehr positiv assoziiert sind und nicht an Krankheit, Sterben oder Tod denken lassen. Dies konnte als eine Strategie verstanden werden, um mit dem Schrecken, den der Begriff „palliativ“ augenscheinlich bei vielen auslöste, umzugehen:

„und ich sehs einfach als, ja Luxussuite bei uns äh wirklich als kleine, als nice to bave" (KPZ 03, Z. 32-33)

Bedingt werden diese Handlungsstrategien des Personals durch eine Unerfahrenheit beim Umgang mit Tod und Sterben. Viele der interviewten Mitarbeiter hatten in ihrem bisherigen Berufsleben wenig Berührung mit sterbenden Patienten und fühlten sich deshalb überfordert mit diesem Thema und drängten auf Fort- und Weiterbildungen: 
„,ich muss natürlich auch sagen dass in meinem ganzen beruflichen Leben und das ist wirklich wenig erst ein Kind bei mir verstorben ist" (KPZ 18, Z. 491-492)

Als eine der Konsequenzen aus der Überforderung ergab sich die Befürchtung vor emotionaler Überlastung und psychischer Überanstrengung, die man durch Gespräche im Kollegenkreis zu bewältigen versuchte:

„ja viele hatten Angst? davoor und und wussten nicht ob sie das schaffen so ein Kind zu betreuen und hatten Ängste das denen das ähmm also emotionaal zu viiiel wird da ham wir bäufig drüber gesprochen " (KPZ 02, Z. 102-104)

Zusätzlich entstand auch die Frage nach dem Wohlsein des „Palliativkindes“. Zum einen war sich das Personal nicht sicher, ob die Patienten der Onkologie in schweren Phasen der Erkrankung oder am Lebensende gerne auf eine andere Station verlegt werden wollen oder ob sie nicht lieber auf der Onkologie verbleiben würden, wo sie Personal und Umgebung kennen:

„möchten die wirklich verlegt werden? (5) mit anderem Personal? andere Umgebung?" (KPZ 03, Z. 571-572)

Dies zeigt viel Einfühlungsvermögen in die Patientensituation.

Zum anderen wurde eine medizinisch kompetente Betreuung der Palliativkinder vom Pflegepersonal angezweifelt, weil die Sorge bestand, dass ärztlicherseits eine unzureichende Ausbildung im schmerztherapeutischen Bereich bestand ${ }^{13}$ :

„,das dieses Kind auch wirklich Schmerzmittel in einer ausreichenden Medikation bekommt und nicht, wir sagen bier manchmal so kleine Dosen die halt nichts bringen weil sie sich halt nicht trauen ähm ordentlich zu dosieren weil sie wenig Erfahrung haben das seh ich halt auch die Versorgung des palliativen Kindes seh ich halt auch eher kritisch weil unsere Ärzte nicht gut ausgebildet sind dafür" (KPZ 01, Z. 125-130)

Zusätzlich waren manche Mitarbeiter nicht der Ansicht, dass das KPZ eine gleichwertige Alternative zu einer Versorgung des Kindes zu Hause oder im Hospiz darstellte - auch dies verdeutlicht die Wissensdefizite des Personals über Inhalte palliativer Versorgung im Allgemeinen und Ziel des KZP im Besonderen:

${ }^{13}$ Hier sei angemerkt, dass in der Klinik mehrere Pädiater angestellt sind, die durch eine Weiterbildung in Palliativ Care die Zusatzbezeichnung „Palliativmedizin“ tragen. Sowohl sie als auch Ärzte der Klinik für Palliativmedizin können und sollen konsiliarisch hinzugezogen werden, sodass eine kompetente medizinische Versorgung der Kinder gewährleistet ist. 
„also ich möchte halt nicht das dieses Palliativzimmer ein Ersatz für ein Hospiz. wird das würd ich ganz schrecklich finden so für die Familie" (KPZ 01, Z. 553-554)

Bei allen Bedenken, die seitens des Personals gegenüber dem KPZ bestehen, gab es doch auch Mitarbeiter, die in der Entstehung des KPZs große Vorteile für sich persönlich und ihre berufliche Zukunft erkannten.

\subsubsection{KPZ als Chance zur Kompetenzerweiterung}

Einige Mitarbeiter sahen im KPZ eine Chance zur persönlichen und beruflichen Kompetenzerweiterung.

In den Interviews zeigte sich, dass sich nur wenige Mitarbeiter in den Entstehungsprozess des KPZs integriert fühlten. Sie hatten nicht das Gefühl, dass die Entscheidung von oben auferlegt war, sondern hatten den Eindruck, dass ihre Meinung erfragt und auch geschätzt wurde:

"und die haben uns im Team immer gefragt was wir denken was wir uns vorstellen"

(KPZ 12, Z. 36-37)

Zusätzlich waren manche Mitarbeiter der Meinung, dass das KPZ einen „Raum“ fürs Sterben schafft. Diese Auffassung teilten Mitarbeiter, die sich in den Entstehungsprozess integriert gefühlt hatten mit jenen, die dies nicht taten. Dieser „Raum“ entstand - nach Auffassung dieser Mitarbeiter - sowohl im wahrsten Sinne des Wortes, indem ein Zimmer entstand, als auch im übertragenen Sinne, dass dem Sterben in der Kinderklinik „Raum“ gegeben wurde und nahmen dies positiv wahr. Bemerkenswert ist, dass beide Perspektiven (sowohl die weiter oben geäußerte negative, wie auch hier die positive) das KZP als Ort des Sterbens wahrnehmen - und dennoch sind gegensätzliche Konnotationen möglich: ,ja weil ich finde dass es halt keinen Raum hatte in der Kinderklinik oder also weder im übertragenen Sinne noch richtig als Raum als Zimmer" (KPZ 10., Z. 447-448)

Beeinflusst wurde diese Wahrnehmung des Zimmers als Chance durch die persönlichen Vorstellungen der Mitarbeiter, was gute Palliativversorgung ausmacht. Dies betraf die medizinische, pflegerische und emotionale Betreuung des Kindes, aber auch die seiner Familie:

„was is für mich Palliativpflege? das bedeutet einfach Zeit für die Eltern zu haben Zeit für den Patienten vielleicht wirklich nur diesen Patienten betreun zu müssen und nich noch fünf andere" (KPZ 18, Z. 275-277) 
In diesem Zitat zeigte sich auch, welch große Bedeutung „Zeit haben“ für die Mitarbeiter hatte. Zeit schien Grundbedingung für eine gute Patientenversorgung und besonders für eine ,gute palliativmedizinische Betreuung zu sein. Dies zeigt sich auch im erhofften höheren Betreuungsschlüssel. Einige Mitarbeiter hielten eine Eins-zu-eins-Betreuung als notwendige Voraussetzung für eine, gute' Palliativversorgung. Zusätzlich wurde deutlich, dass der Elternbetreuung ein großer Stellenwert in der Kinderpalliativmedizin zukommt. Dies ist ganz im Sinne der WHO-Definition für Palliativmedizin im Kindesalter (vgl. Kapitel 3 Stand der Forschung). Einige der Mitarbeiter, die das KPZ als Chance zur Kompetenzerweiterung ansahen, hatten bereits vor der Eröffnung des KPZ ein Interesse an Palliativmedizin und entschlossen sich z. B. schon vor Beginn der Planungsphase eine Weiterbildung zu absolvieren. Sie sahen in dieser Weiterbildung eine Möglichkeit, die eigenen fachlichen Kompetenzen zu erweitern, ihre Fähigkeiten zu verbessern und auch für sich selbst den Umgang mit sterbenden Patienten zu erleichtern. Unabhängig vom KPZ sahen diese Mitarbeiter damals eine fachliche Weiterbildung als Möglichkeit, die Arbeit mit sterbenden Kindern für sich selbst subjektiv zu vereinfachen. Interessanterweise erwarten jene Mitarbeiter, die durch das KPZ eine verstärkte Konfrontation mit sterbenden Patienten befürchten, die gleiche subjektive Verringerung der emotionalen Beanspruchung durch eine fachliche Weiterbildung:

„in den letaten Jahren waren 3 Patienten verstorben auch und dann hab ich so gedacht Mensch das interessiert mich schon wie man dann auch so weiter damit umgeht oder besser damit umgehen kann einfach" (KPZ 16, Z. 109-112)

Einige Mitarbeiter, die zuvor nicht den Bedarf einer Weiterbildung in Palliative Care gesehen hatten, betrachteten das KPZ als Impuls dafür, ihre Fähigkeiten in diesem Bereich zu verbessern und etwas Neues zu erlernen. Bei diesen schien es, als hätte sie das KPZ erst auf die Idee gebracht, dass Kinderpalliativmedizin ein mögliches und nun nach KPZ Eröffnung ein sinnvolles Weiterbildungsgebiet darstellt:

$$
\begin{aligned}
& \text { „ja vorher wär ich nich auf die Idee gekommen ne Palliativweiterbildung zu machen, } \\
& \text { warum ((lacht)) (KPZ 17, Z. 285-286) }
\end{aligned}
$$

Man kann also im Zusammenhang von dem Bedürfnis nach fachlicher Weiterbildung von drei Gruppen von Mitarbeitern sprechen: Die erste Gruppe interessierte sich, unabhängig vom KPZ, für dieses Fachgebiet und sah durch die tägliche Arbeit mit schwerstkranken und zuweilen sterbenden Kindern einen persönlichen Bedarf, solch eine Fortbildung zu absolvieren. Die zweite Gruppe hatte vor Initiierung des KPZs keinen Bedarf für eine persönliche Weiterbildung im Bereich Palliativmedizin gesehen. Die neuen 
Arbeitsanforderungen, die das KPZ mit sich brachte, verursachten bei ihnen allerdings Unsicherheit und Überforderung. Als Ausweg sahen diese Mitarbeiter nun eine fachliche Fortbildung. Die dritte Gruppe von Mitarbeitern schien vor Initiierung nie den Gedanken an eine entsprechende Weiterbildung gehabt zu haben, empfand das KPZ aber nun als einen Anreiz. Hier wurde es weniger als ein Bewältigungsmechanismus für Überforderung dargestellt, sondern vielmehr als eine sinnvolle Maßnahme. Dies mag auf einen rationalen und sachlicheren Umgang mit der eigenen empfundenen Überforderung zurückzuführen sein. Es lässt sich aber festhalten, dass in fast allen Interviews das Bedürfnis nach fachlicher Weiterbildung thematisiert wurde. Entweder war dieses bereits befriedigt worden oder die Interviewpartner drängten darauf. Das Thema Weiterbildung ist aber auf jeden Fall von großer Bedeutung für die befragten Mitarbeiter.

Für diese Lösungsstrategie existierten verschiedene Vorbedingungen: Zum einen erfolgte eine Anerkennung der palliativen Versorgung von Kindern von außen. Besonderen Einfluss hatte hier die mediale Präsenz, die das Kinderpalliativzimmer im Raum Göttingen während seiner Entstehung hatte. Es fanden öffentliche Spendenaktionen, z. B. in Supermärkten und Spielzeuggeschäften statt. Innerhalb der Klinik wurden Informationsplakate aufgehängt. Diese öffentliche Präsenz regte einerseits dazu an, sich mit dem Thema auseinanderzusetzen. Zum anderen hatten sie das Gefühl, als Mitarbeiter der beteiligten Station somit auch eine gewisse Aufmerksamkeit und Wichtigkeit zu erlangen:

„genau dann hab ich natürlich auch viel in der Öffentlichkeit gesehn dass gesammelt wurde dafür in Rewe Märkten und so wo das balt aushing" (KPZ 14, Z. 21-23)

Zusätzlich erlebte die Kinderpalliativmedizin in den vergangenen Jahren einen „Boom“, wie ein Interviewpartner anmerkte. Damit bezog dieser sich darauf, dass in diesem Bereich in den letzten Jahren viele Entwicklungen stattgefunden hatten (vgl. Kapitel 3 Stand der Forschung) und Kinderpalliativmedizin sehr viel stärker ins Bewusstsein der Bevölkerung gelangt war:

$$
\begin{aligned}
& \text { „ich glaub das Thema mehr in aller Munde und zunehmend stellen sich viele auch die } \\
& \text { Fraagen? und dann hat man jetzt den Vorteil dass man Leute hat die man ansprechen } \\
& \text { kann dies dann mit helfen umzusetzen und das gabs halt vor fünfzehn Jahren noch nich } \\
& \text { da hat sich was getaan" (KPZ 12, Z. 544-548) }
\end{aligned}
$$

Die Begleitung eines Menschen während des Sterbens wurde von manchen Mitarbeitern als eine Form der Hilfe und Nächstenliebe wahrgenommen. Erledigte man diese Aufgabe ,gut', d. h. zur Zufriedenheit des Patienten bzw. seiner Angehörigen, so leistete man einen 
wichtigen Betrag zum Leben dieser Menschen. Einige Mitarbeiter empfanden es auch als „Privileg“, Menschen beim Sterben begleiten und unterstützen zu dürfen:

„,das sind ja sehr existentielle Dinge den Tod eines Menschen miterleben die Trauer der Angehörigen und so und das ist natürlich emotional auch sehr anspruchsvoll und auch sehr traurig und gleichzeitig hab ichs wie soll es immer als ne Art Privileg empfunden in so existentiellen Situationen in die Familien involviert sein zu dürfen also so daran teilhaben zu dürfen" (KPZ 07, Z. 394-399)

Durch dieses ,Privilegiert-Sein` hatten diese Mitarbeiter das Gefühl, sich von der Masse abzuheben und etwas ,Besonderes` zu sein bzw. eine Sonderstellung in der Alltagsroutine zu genießen. Diese Mitarbeiter waren der Meinung, dass sich Sterbebegleitung vorteilshaft auf ihren Charakter auswirkte und sie im positiven Sinne prägte:

„ich habe gemerkt das es mich einfach auch charakterlich hat reifen lassen, man reift daran ja auch man entwickelt sich damit ja auch" (KPZ 07, Z. 478 479)

Es zeigte sich, dass eine Palliativweiterbildung die Berufsinterpretation der Mitarbeiter scheinbar stärken konnte, weil dadurch Handlungsweisen bestätigt werden konnten. Manche Arbeitsweisen, wie sie z. T. seit Jahren praktiziert wurden, konnten im Rahmen einer Weiterbildung als richtige Handlungsweise bejaht werden:

„einfach vielleicht auch so ne Bestätigung von dem was man vielleicht schon immer unter Patientenversorgung und auch unter Palliativversorgung verstebt" (KPZ 18, Z. 104-105)

Des Weiteren bestand bei einigen Mitarbeitern die Hoffnung, dass der Kinderpalliativbereich an der UMG weiter ausgebaut werden würde. Es existierten unter den Mitarbeitern verschiedene Visionen, wie dieser Ausbau aussehen könnte: Manche sahen eine eigene abgetrennte Kinderpalliativstation als erstrebenswertes Ziel. Einzelne hofften auf eine Vergrößerung des Bereiches, jedoch weiterhin integriert in den momentanen stationären Kontext:

„ich hoffe auch dass sich das weiter entwickelt dass es vielleicht noch en zweites Zimmer gibt und vielleicht noch en drittes und dass sich daraus über die Jahre vielleicht auch ne Station Palliativstatioon entwickelt, aber das muss wachsen das kann man nich irgendwie so aus dem Boden stampfen so das muss wachsen, und, ich glaub das is ne ganz, gute Keimzelle jetżt dieses Zimmer und hoffe dass das weiter das gut angenommen wird und weiter wächst" (KPZ 05, Z. 442-447)

Und es setzen auch viele Mitarbeiter - besonders aus dem Pflegebereich - ihre Hoffnung in die ärztliche Behandlung der Kinder im Palliativzimmer durch speziell weitergebildete 
Pädiater. Diese sollten die medizinische Betreuung der Kinder von den Stationsärzten übernehmen:

„und was noch ne Veränderung sein wird, die ich mir erhoffe? die babnt sich an aber ich bin noch nicht sicher ob sich das durchsetzt ist eben das diese Palliatiivkinder ärəttlich von den Palliatiivärzten betreut werden und nicht vom Stationsoberarz̨" (KPZ 07, Z. 1237-1240)

Dies schien in direktem Zusammenhang zu stehen mit den Uneinigkeiten zwischen Ärzten und Pflegenden bei Therapieentscheidungen (vgl. Kapitel 5.7.2 Intra- und interprofessionelle Konflikte belasten Zusammenarbeit) und der angezweifelten Kompetenz der Stationsärzte seitens des Pflegepersonals (vgl. Kapitel 5.8.1 Überforderung und Selbstunsicherheit). 


\section{Diskussion}

In dieser Studie wurde untersucht, wie der Implementierungsprozess eines Kinderpalliativzimmers in der Klinik für Kinder- und Jugendmedizin Göttingen von den beteiligten Mitarbeitern erlebt wurde und welche Erwartungen sie an das Zimmer hatten. Es sei nochmals darauf hingewiesen, dass diese Studie sich nicht zum Ziel gesetzt hatte zu untersuchen, welche Vorteile ein KPZ als Versorgungsstrukturerweiterung für Kinder mit LBE und LLE bringt. Es sollte vielmehr erfahren werden, wie das Personal die Entstehung des KPZs erlebt hat.

Die Befragung der Mitarbeiter ergab, dass manche Gegebenheiten während der Planung und Entstehung des Zimmers verhaltene erste Reaktionen der Interviewpartner auf das KPZ zur Folge hatten. Die Implementierung des KPZs auf der Station Neuropädiatrie der UMG führte bei den Mitarbeitern zur gefühlten Entstehung einer „Klassengesellschaft“ auf der Station. Verursacht wurde diese Wahrnehmung vor allem durch organisatorische Schwierigkeiten im Rahmen der Planung und Entstehung des Zimmers. Aber auch die beschriebene Abgrenzungsproblematik des Aufgaben- und Verantwortungsbereiches der einzelnen Mitarbeiter spielte eine Rolle für die subjektive Entstehung einer „Klassengesellschaft“. Aufgrund dieser Empfindung reagierte ein Großteil der befragten Mitarbeiter distanziert auf das KPZ während seiner Entstehungs- und Anlaufphase. Bedingt wurde die Distanzierung durch eine fehlende Einbeziehung des Personals in den Implementierungsprozess und der Herausforderung, die der Kontakt mit sterbenden Patienten als solcher darstellte. Es kam bei einigen Mitarbeitern zu einem Gefühl der Überforderung, aber manche sahen das KPZ auch als Chance zur eigenen Kompetenzerweiterung.

\subsection{Gefühl einer entstandenen „Klassengesellschaft“ und Zwei- Klassen-Medizin}

Mit der Errichtung des KPZs wurde ein schöner Raum in der Klinik für Kinder- und Jugendmedizin Göttingen geschaffen, der für schwerkranke Kinder und ihre Familien eine Verbesserung der stationären palliativmedizinischen Versorgung darstellen kann. Da jedoch nur ein Stationszimmer aufwändig renoviert wurde und die Ausstattungsunterschiede signifikant sind, resultierte eine Ungleichheit bei der Patientenunterbringung auf der Station. Eine Interviewpartnerin/ein Interviewpartner beschrieb dies mit dem Begriff 
„Klassengesellschaft auf der eigenen Station“ (vgl. Kapitel 5.3 Kernkategorie: Durch KPZ entsteht „Klassengesellschaft“ auf der Station, S. 38). Auch in zwei weiteren Interviews tauchte der „Klassen-Begriff“ auf. Hier war von „Zwei-Klassen-Station“ bzw. „erster Klasse und zweiter Klasse“ die Rede. Die Verwendung dieser Bezeichnung scheint im vorliegenden Zusammenhang ungewöhnlich. Es existieren viele verschiedene Definitionen des Klassen-Begriffs in der Literatur und die wohl bekannteste Definition sei beispielhaft angeführt: Der Begriff Klasse beschreibt Menschen, die sich aufgrund gemeinsamer ökonomischer Merkmale in einer vergleichbaren sozialen Lage befinden. Die ähnliche ökonomische Situation hat ein „Gemeinsamkeitsgefühl“ zur Folge. Karl Marx bezeichnete dies als „Klassenbewusstsein“. In einer „Klassengesellschaft“ besteht aufgrund „fehlender Mobilitätschancen zum Aufstieg in höhere Statusgruppen eine weitgehende „Geschlossenheit“ des sozialen Ungleichheitsgefüges“ (Korte 2000, S. 182).

In der Bundesrepublik Deutschland taucht der Klassen-Begriff im medizinischen Kontext auf, wenn von einer Zwei-Klassen-Mediz̨in die Rede ist. Dieses politische Schlagwort löst in der Gesellschaft Diskussionen aus. Der - nicht präzise definierte - Begriff beschreibt eine medizinische Versorgung, deren Qualität scheinbar von der Krankenversicherung (gesetzliche Krankenversicherung [GKV] oder privaten Krankenversicherung [PKV]) des Patienten abhängig ist. Frank Schulze Ehring und Christian Weber erklären die negative öffentliche Wahrnehmung des Begriffs mit der Angst des Einzelnen, „zukünftig im Bedarfsfall nicht mehr das Notwendige, sondern nur noch das Notwendigste zu bekommen“ (Schulze Ehring und Weber 2009, S. 140). Bedingung für das Bestehen einer Zwei-Klassen-Mediz̨in wäre eine Leistungs- und Qualitätsdifferenzierung, welche zu Lasten Dritter gehen würde. Dies würde bedeuten, dass die Entscheidung einer Bevölkerungsgruppe (PKV-Versicherte) für eine erstklassige medizinische Versorgung dazu führen würde, dass andere (GKV-Versicherte), für die diese Behandlung keine Option darstellte bzw. darstellen würde, eine schlechtere medizinische Versorgung erhalten würden. Eine solche Differenzierung zu Lasten Dritter ist - nach Meinung der Autoren in Deutschland nicht gegeben (Schulze Ehring und Weber 2009). Eine gegensätzliche Meinung vertritt der Autor Karl Lauterbach (Lauterbach 2008).

Die vorangegangenen Begriffserläuterungen lassen vermuten, dass die befragten Mitarbeiter mit der Verwendung des Klassen-Begriffes weder unterschiedliche ökonomische Merkmale von Patienten, noch eine differierende medizinische Versorgung aufgrund der Krankenversicherung der Patienten beschreiben wollten. Das KPZ wurde nicht für PKV-Patienten, sondern für Kinder und Jugendliche mit lebenslimitierenden Erkrankungen zur Behandlung von belastenden körperlichen Symptomen oder 
psychosozialen Belastungen, bei akuter Symptomverschlechterung oder am Lebensende initiiert. Im Folgenden soll deshalb ein anderer Erklärungsansatz für die Verwendung des Klassen-Begriffs im Zusammenhang mit dem KPZ gegeben werden:

\subsubsection{Die Antizipation von Neid}

Eine Unterbringung im KPZ liefert sehr gute räumliche Rahmenbedingungen für die stationäre Versorgung eines lebensbedrohlich oder lebenslimitierend erkrankten Kindes. Es mag zu einer ,Besserbehandlung“ von Kindern und Jugendlichen in schwierigen Phasen ihrer Erkrankung oder am Lebensende kommen. Es muss an dieser Stelle differenziert werden zwischen einer Besserbehandlung im Sinne des Genusses eines größeren und umfangreich ausgestatteten Zimmers und einer medizinischen Vorzugsversorgung. Es gibt keine objektiven Hinweise, dass Kinder im KPZ eine bessere medizinische Behandlung bekommen, als in jedem anderen Zimmer der UMG. Dies ist auch nicht der Anspruch oder das Ziel des KPZs. Es gab jedoch einzelne Mitarbeiter, die eine unbewusste Vorzugsbehandlung der Patienten im KPZ für möglich hielten (vgl. 5.7.3 Sterben als professionelle Herausforderung S. 64). Aus den ungleichen Rahmenbedingungen für die Patienten der Station entstand bei einigen Mitarbeitern die Befürchtung, dass Neid auf die Unterbringung im KPZ bei den Eltern entstehen könnte, deren Kinder nicht im KPZ versorgt werden und es deshalb zu Konflikten auf der Station kommen könnte. Die Emotion Neid wird in der Literatur vielfach beschrieben und untersucht. Sie beschreibt die Empfindung, die entsteht, wenn man sich mit Anderen vergleicht und feststellt, dass jene etwas besitzen was einem selbst fehlt und man auch sein Eigen nennen möchte (Parrott und Smith 1993; Smith et al. 1996). Menschen empfinden vor allem Neid, wenn sie sich mit Personen vergleichen, die ihnen ähnlich sind (Smith 2008). In der Literatur zeigte sich Neid auf Statussymbole (z. B. Sportwagen, Designerhandtaschen etc.) oder Eigenschaften (z. B. Schönheit, Intelligenz, Humor) einer anderen Person (Bucher 2012). Georg Foster kam zu dem Ergebnis, dass die meisten Menschen Neid nicht offen zugeben wollen. Zu diesem Schluss kam er durch eine Befragung von Studenten, bei der 50\% der Studienteilnehmer angaben nie neidisch zu sein. Seine Neid-Forschung ergab jedoch, dass der Neid in jeder Gesellschaft und bei jedem Menschen existent ist (Foster 1972). In der vorliegenden Studie schien einigen Mitarbeitern der Neid bei Eltern von Patienten wahrscheinlich zu sein, v. a. als Reaktion auf die - als ungerecht empfundene Zimmerausstattung (vgl. 5.5.2 Abgrenzungsproblematik des Aufgaben- und Verantwortungsbereiches, S. 51). Würde es sich beim KPZ um ein renoviertes Zimmer ohne den bestehenden palliativen Versorgungsanspruch handeln, so wäre es durchaus 
vorstellbar, dass Eltern die stationäre Unterbringung ihres Kindes in einem regulären Zimmer mit der eines Patienten im KPZ vergleichen und auf die wahrgenommenen Unterschiede neidisch reagieren könnten. Im vorliegenden Fall wurde jedoch Neid von Eltern auf die Privilegien, die ein lebenslimitierend erkranktes Kind genießen darf, von einigen Mitarbeitern antizipiert. Für die Unterbringung im KPZ und den Genuss der Zimmerausstattung liegt meist eine Erkrankung vor, die zu einem vorzeitigen Tod führen wird. Die Verknüpfung dieser Faktoren: Das Kind leidet an einer lebensbegrenzenden Erkrankung, befindet sich in einer Krankheitskrise und wird deshalb in einem Zimmer mit besseren räumlichen Rahmenbedingungen versorgt, kann schwerlich als beneidenswertes Statussymbol aufgefasst werden. Die vermeintliche Entstehung von Neid scheint deshalb an dieser Stelle ungewöhnlich. Es ist wichtig, sich vor Augen zu führen, dass jene Mitarbeiter annahmen bzw. befürchteten, dass Eltern, deren Kinder in einem regulären Stationszimmer untergebracht sind, neidisch reagieren und eine Verlegung ihres eigenen Kindes ins KPZ fordern könnten. Ob bei den besagten Eltern tatsächlich Neid entstehen würde, war zum Zeitpunkt der Interviews noch offen und konnte in der vorliegenden Studie nicht festgestellt werden, da keine Befragung der Eltern stattfand. Die geschilderte Szene der Verlegung eines Kindes ins KPZ (vgl. 5.6.1 Ablehnung des KPZs, S. 55) zeigte allerdings keine Freude bei den Eltern, wie es zu erwarten gewesen wäre, wenn das KPZ tatsächlich, wie angenommen, das ,Objekt der Begierde` wäre. Die betreffenden Eltern reagierten vielmehr abwehrend und ängstlich und mussten zu einer Verlegung geradezu überredet werden. Erst die Aufklärung durch die Mitarbeiter konnte ihnen verdeutlichen, was der Anspruch und das Ziel des Göttinger KPZs ist. Hier wird deutlich, wie wichtig gut informierte Mitarbeiter sind, damit kompetente Aufklärungsarbeit geleistet werden kann. Für die Eltern in der beschriebenen Szene war die Angst, dass eine Verlegung ihres Kindes ins KPZ mit dessen Sterben gleichzusetzen sei, so groß, dass sie sich nicht über die Aussicht auf ein besser ausgestattetes Zimmer freuten. Trotz dieses Erlebnisses schien es zu einer Identifikation von vielen Mitarbeitern mit den Eltern zu kommen, deren Kinder nicht im KPZ untergebracht waren und dem Gefühl, dass diese Familien mit der Unterbringung in einem regulären Zimmer ungerecht behandelt werden würden. Einen möglichen Erklärungsansatz für dieses Empfinden bei den Mitarbeitern liefert Edmond Cahn: Der Mensch empfindet Unrecht, welches anderen wiederfährt, als einen Angriff auf sich selbst (Cahn 1964). Fraglich bleibt, ob man an dieser Stelle von Ungerechtigkeit sprechen kann, wenn Patienten in den regulären Zimmern eines Krankenhauses untergebracht werden. Es stellt sich weiterhin die Frage, weshalb die Ausstattungsunterschiede zwischen dem KPZ und den regulären Stationszimmern so viel 
Raum in den Interviews einnahmen und der Begriff „Klassengesellschaft“ verwendet wurde. Es könnte sich bei den wiederholten Verweisen auf die Ansichten von Eltern um Vorwände der Mitarbeiter handeln. Es schien bei vielen Mitarbeitern eine Unzufriedenheit z. B. über die subjektive fehlende Einbeziehung in den Entstehungsprozess des Zimmers und die unzureichende Information über das Konzept des KPZs und die neuen Arbeitsanforderungen $\mathrm{zu}$ herrschen. Die vermeintlichen Bedenken Anderer (Eltern) anzuführen, könnte eine vordergründige Begründung für die eigene ablehnende Haltung gegenüber dem KPZ und die eigene Kritik am Implementierungsprozess darstellen. So könnten es die besagten Mitarbeiter als einfacher empfinden, vermeintliche Bedenken von Angehörigen und sich entwickelnde Konflikte auf der Station zu problematisieren, als über ihre eigene Unzufriedenheit am Arbeitsplatz zu sprechen. Somit wäre die gefühlte Entstehung einer „Klassengesellschaft“ auf der Station eine Beschreibung der Unzufriedenheit der Mitarbeiter mit Aspekten des Implementierungsprozesses des KPZs.

\subsection{Ressourcenmangel als große Herausforderung}

Bei der Interviewauswertung offenbarte sich zusätzlich ein subjektiver Ressourcenmangel in der Klinik für Kinder- und Jugendmedizin Göttingen, der organisatorische Schwierigkeiten verursachte. Besonders hervorzuheben sei an dieser Stelle der beschriebene Personalmangel im Pflegebereich. Die Mitarbeiter schilderten hier eine gefühlte personelle Unterbesetzung. Diese, teils schon vor Implementierung des KPZs bestehenden Engpässe, wurden - subjektiv - durch das KPZ verstärkt. Das Pflegepersonal fühlte sich für eine adäquate Patientenversorgung nicht ausreichend besetzt. $\mathrm{Da}$ das KPZ die Arbeitsanforderungen in den Augen vieler Mitarbeiter erhöhte, aber die Station nur eine zusätzliche Stelle bekam, schien dem Pflegepersonal eine Kompensation des gestiegenen Arbeitsaufwandes nicht möglich. In der Literatur zeigt sich, dass eine personelle Unterbesetzung gerade im Krankenpflegebereich ein großes Problem ist Janiszewski Goodin 2003; Hassmiller und Cozine 2006), womit Krankenhäuser weltweit zu kämpfen haben (Booth 2002). Immer weniger junge Frauen und Männer entscheiden sich für eine Ausbildung in der Gesundheits- und Krankenpflege. Ursächlich hierfür könnte das schlechte Image sein, mit dem die Gesundheits- und Krankenpflege zu kämpfen hat („Frauenberuf“, geringe Karrierechancen, harte körperliche Arbeit, schlechte Bezahlung u. ä.) , weshalb sich heute weniger junge Menschen für diese Ausbildung entscheiden (Hemsley-Brown und Foskett 1999; Janiszewski Goodin 2003). Diese Entwicklung betrifft auch die UMG. Es stellte sich nämlich als schwierig heraus, die neu geschaffene Stelle zu 
besetzen. In den Interviews wurde deutlich, dass auch die Mitarbeiter in Göttingen mit einem hohen Arbeitspensum und personeller Unterbesetzung zu kämpfen hatten (vgl. Kapitel 5.5.1 KPZ als organisatorische Herausforderung, S. 48). Es schien, dass sich das Personal eine Optimierung und Anpassung der Ressourcen gewünscht hätte, bevor der neue Behandlungsschwerpunkt Kinderpalliativmedizin initiiert wurde. Es konnte im Rahmen dieser Studie nicht geklärt werden, ob die Bewältigung der Arbeitsanforderungen mit dem vorhandenen Personal möglich oder ob eine Ergänzung des Personals notwendig ist. Dies sollte überprüft werden, um im Zweifel eine Überlastung von Mitarbeitern zu verhindern.

\subsection{Rufbereitschaft als Hindernis}

Ein Grund, warum manche Mitarbeiter aus dem Pflegebereich ablehnend auf das KPZ reagierten, war die Umstrukturierung des ambulanten Palliativdienstes für Kinder in Göttingen. Auch Mitarbeiter aus der Gesundheits- und Kinderkrankenpflege sollten fortan an der spezialisierten ambulanten Palliativversorgung mitwirken (vgl. 5.6.1 Ablehnung des KPZs). In der Literatur zeigte sich, dass Rufbereitschaft bei Mitarbeitern Schlafstörungen und eine Beeinträchtigung des Privatlebens verursachen kann, was wiederum zu einer Verringerung der Arbeitszufriedenheit, einer Senkung der Arbeitsfähigkeit und zu großer Erschöpfung führen kann (Heponiemi et al. 2014). Auch viele der befragten Mitarbeiter lehnten eine Teilnahme am ambulanten Palliativrufdienst ab, weil sie durch ihn besonders Beeinträchtigungen in ihrem Privatleben befürchteten, wie z. B. weniger Zeit mit ihrer Familie oder Störung bei Freizeitaktivitäten. Es zeigte sich, dass nach 18 Monaten KPZ der ambulante 24-Stunden-Rufdienst von vier Mitarbeitern bewältigt werden muss. Darin ist abzulesen, dass die Ablehnung bezüglich dieses Dienstes und der damit verbundenen Rufbereitschaft scheinbar nicht abgenommen hat. Die Aufrechterhaltung eines 24Stunden-Rufdienstes mit vier Mitarbeitern bedeutet eine sehr starke Belastung der Beteiligten. Langfristig kann dieses Konzept mit dieser geringen Teilnahmezahl nicht aufrechterhalten werden, ohne dass eine Überlastung der Akteure befürchtet werden muss.

\subsection{Fehlende Integration des Personals}

Ein großes Problem stellte der empfundene Ausschluss des Personals vom Entstehungsprozess des KPZs dar. Dies wurde in den meisten Interviews thematisiert und scheint die distanzierte Haltung gegenüber des KPZs maßgeblich beeinflusst zu haben. Stefanie Humburg identifizierte unzureichende Information und einen fehlenden Überblick 
über Zusammenhänge als Ursachen für Konfliktentstehung bei Krankenhausmitarbeitern. Ihrer Ansicht nach ist die Befriedigung des Informationsbedürfnisses entscheidend, um Ängste zu bekämpfen und dem einzelnen Mitarbeiter Verhaltenssicherheit zu geben (Humburg 2001). Auch Leuzinger und Luterbacher kamen zu dem Ergebnis, dass fehlende Information der Mitarbeiter zu Ungewissheit, Verwirrungen, Misstrauen und Angst führen kann. Ihre Beobachtungen zeigten weiterhin, dass der Informationsfluss in Krankenhäusern - aufgrund der hierarchischen Strukturen - gehäuft von oben nach unten stattfindet. Erfolgt der Informationsfluss über mehrere Stufen, so besteht die Tendenz, dass v. a. Hintergrundinformationen auf einzelnen Stufen zurückgehalten werden und so nicht jeden Mitarbeiter erreichen (Leuzinger und Luterbacher 2000). Dies war auch im Rahmen der Implementierung des KPZ an der UMG zu beobachten. Viele Mitarbeiter reagierten verunsichert oder verärgert, weil sie subjektiv über zu wenig Informationen über das KPZ und die neuen inhaltlichen Arbeitsanforderungen verfügten. Es entstand allerdings der Eindruck, dass wenige Mitarbeiter Eigeninitiative zeigten, um Informationen bezüglich des KPZs zu erhalten. Zu diesem Thema führen Leuzinger und Luterbacher (2000) an, dass dem Arbeitgeber nicht die alleinige ,Bringschuld‘ zugeschoben werden darf. Der einzelne Mitarbeiter ist auch in der Verpflichtung, sich zu informieren und darf nicht nur darauf warten, Informationen zu erhalten. Eine Informiertheit über wichtige Belange liegt somit im Verantwortungsbereich aller Beteiligten. Jene Mitarbeiter, die das Gefühl unzureichender Informiertheit hatten, reagierten mit Kritik an der Umsetzung des Zimmers und wurden zunehmend skeptisch gegenüber dem Projekt KPZ. Wenige Mitarbeiter fühlten sich vom Entstehungsprozess nicht ausgeschlossen und äußerten weniger Kritik bezüglich des KPZs. Es wurde deutlich, dass die Verärgerung über den Ausschluss besonders bei den Mitarbeitern der Neuropädiatrie zu beobachten war. Durch die Verortung des KPZs auf der eigenen Station schien ihnen eine lückenlose Informationsweitergabe deutlich wichtiger zu sein als jenen Mitarbeitern, die nicht auf dieser Station arbeiteten.

\subsection{Arbeit mit Palliativkindern kann belastend sein}

Es zeigte sich, dass die aus der gefühlten „Klassengesellschaft“ resultierende Überforderung und Selbstunsicherheit eine große Rolle für das Erleben des Implementierungsprozesses durch die Mitarbeiter spielte. Von besonderer Bedeutung schien die Befürchtung vor emotionaler Überlastung zu sein. Die Arbeit mit sterbenden Patienten und ihren Angehörigen ist für Mitarbeiter sehr fordernd. Zahlreiche Studien 
zeigen, wie groß gerade die psychische Beanspruchung von Mitarbeitern der Kinderonkologie oder der Kinderintensivstation ist (Spencer 1994; Davies et al. 1996; Granek et al. 2015). Die psychische Belastung kann im Burnout enden (Whippen und Canellos 1991). Granek et al. (2015) kamen zu dem Ergebnis, dass die Mitarbeiter nach dem Tod eines Patienten mit Trauerreaktionen (z. B. Traurigkeit, Schlaflosigkeit, Gefühl eines persönlichen Verlustes) zu kämpfen hatten, aber auch Auswirkungen auf das Privatleben und die Arbeit konnten die Folge sein. Die Studie beschäftigte sich mit Kinderonkologen, die im Schnitt mit 1,5 Todesfällen pro Monat konfrontiert waren. Dies ist eine deutlich stärkere Konfrontation, als es in der Abteilung Neuropädiatrie der UMG der Fall ist. Doch viele Mitarbeiter befürchteten eine deutliche Zunahme der Sterbefälle in ihrem Berufsalltag durch das KPZ und sahen genau diese Formen der psychischen Überlastung durch das Zimmer auf sich zukommen. Betrachtet man die Patientenstatistik nach 18 Monaten Nutzung des KPZs an der UMG, so zeigt sich, dass die befürchtete stärkere Konfrontation mit sterbenden Patienten nicht eingetreten ist. Es verstarb in diesem Zeitraum ein Kind im KPZ. Löste vor allem die Befürchtung vor einer zunehmend erforderlichen Auseinandersetzung mit sterbenden Patienten die Überforderung aus, so ist es möglich, dass die Überforderung aufgrund der nicht eintreffenden Annahme bereits nachgelassen hat. Darüber könnte nur eine erneute Mitarbeiterbefragung Auskunft geben.

\subsection{Begriffsunsicherheit unter den Mitarbeitern}

Es stellte sich heraus, dass bei vielen Mitarbeitern eine große Unsicherheit bezüglich der Aufgaben und Ziele von Palliativmedizin bestand. Welche Patienten eine palliativmedizinische Versorgung benötigen, schien für viele Mitarbeitern kaum zu beantworten zu sein (vgl. Kapitel 5.8.1 Überforderung und Selbstunsicherheit). Dies mag darauf zurück zu führen sein, dass Beschreibungen, Definitionen und Begrifflichkeiten sehr unterschiedlich verwendet werden. Die European Association for Palliative Care (EAPC) Task Force kam zu dem Ergebnis, dass in Europa eine sehr heterogene Terminologie herrscht (Centeno et al. 2007). Zusätzlich sind die Definitionen verschiedener Fachgesellschaften nicht deckungsgleich. Einige Definitionen seien im Folgenden angeführt: Laut WHODefinition für Palliative Care dient Palliativmedizin dazu, die Lebensqualität von Patienten und ihren Familien, die mit Problemen konfrontiert sind, welche mit einer lebensbedrohlichen Erkrankung verbunden sind, zu verbessern (WHO Definition of Palliative Care). Die WHO-Definition beinhaltet außerdem, dass Schmerzlinderung und die Behandlung belastender Symptome im Mittelpunkt stehen. Eine derartige Behandlung 
sollte - so fordert es die WHO - frühzeitig ins Therapiekonzept integriert werden. Die Deutsche Gesellschaft für Palliativmedizin (DGP) definiert Palliativmedizin ähnlich und doch werden Unterschiede deutlich: Es ist von „Patienten mit einer nicht heilbaren progredienten und weit fortgeschrittenen Erkrankung mit begrenzter Lebenserwartung“ die Rede. In dieser Definition wird, mit dem Verweis auf „weit fortgeschrittene Erkrankung“ und „begrenzte Lebenserwartung“, ein engerer zeitlicher Rahmen abgesteckt. Zwar fordert auch die DGP eine frühzeitige Integration der Palliativmedizin ins Therapiekonzept, auch begleitend zur kausalen Therapie, aber es kann der Eindruck entstehen, dass die Palliativmedizin vor allem am Lebensende zum Einsatz kommt (Radbruch et al. 2005, S. 16). Das Bundesgesundheitsministerium versteht unter einer Palliativversorgung eine „Versorgung schwerstkranker und sterbender Menschen“. In dieser Definition werden sterbende Patienten als Zielgruppe explizit genannt. Untersuchungen zeigen ferner, dass in Europa besonders Erwachsene mit fortgeschrittenen Tumorerkrankungen eine Palliativversorgung erhalten (Radbruch und Payne 2011). Die erwähnten Definitionen können eine Erklärung liefern, warum Palliativmedizin von vielen Mitarbeitern mit sterbenden Patienten assoziiert und am Lebensende verortet wurde. Diese Vorstellungen von Palliativmedizin schienen dann auf die pädiatrische Palliativversorgung übertragen worden zu sein. Das Spektrum an Erkrankungen, die eine Palliativversorgung bei Kindern erforderlich macht, ist deutlich heterogener als bei Erwachsenen (vgl. Kapitel 2 Einleitung). Nicht-onkologische Erkrankungen machen einen höheren Prozentsatz als in der Palliativmedizin für Erwachsene aus (vgl. Kapitel 3.3 Unterschiede in der Palliativversorgung von Erwachsenen und Kindern). Viele betroffene Kinder und Jugendliche leiden über viele Jahre an ihrer Erkrankung und auch für sie fordert die WHO eine Palliativversorgung ab Diagnosestellung. Viele Göttinger Mitarbeiter verstanden unter einem „Palliativkind“ jenes, bei dem das Sterben unmittelbar bevorstand. Diese, in den Köpfen der Mitarbeiter, irrtümliche Begriffsdefinition spielt eine große Rolle bei der Mitarbeiterüberforderung und erschwerte scheinbar auch die Auswahl, welches Kind ins KPZ verlegt werden soll. Hier scheint Aufklärungsarbeit bei den Mitarbeitern erforderlich zu sein.

\subsection{Fachliche Weiterbildung als Ausweg}

In den Interviews zeigte sich auch, welchen großen Stellenwert eine fachliche Weiterbildung als Mittel gegen Überforderung hat. In der Literatur finden sich verschiedene Kompetenzen, die das Pflegepersonal für die Pflege am Lebensende 
bedeutsam findet. In einer amerikanischen Studie $\mathrm{zu}$ diesem Thema wurden Symptommanagement, Kommunikation über Tod und Sterben mit Patient und Angehörigen sowie Aufgaben und Ziele der Palliativmedizin zu kennen, als wichtigste Kompetenzen genannt (White et al. 2001). Nahezu alle Göttinger Mitarbeiter, die sich überfordert fühlten, sahen vor allem in einer fachlichen Weiterbildung eine Lösungsmöglichkeit (vgl. Kapitel 5.8.1 Überforderung und Selbstunsicherheit). Die befragten Mitarbeiter hatten das Bedürfnis, ihre Kommunikationsfähigkeiten bezüglich Tod und Sterben mit Patienten und Angehörigen zu verbessern. Symptom- und besonders Schmerzkontrolle wurden in den Interviews thematisiert. Hier sahen besonders die befragten Pflegekräfte Verbesserungsbedarf. Allerdings verorteten sie Schmerzkontrolle im Kompetenzbereich der Ärzte und sahen hier noch Weiterbildungsbedarf (vgl. Kapitel 5.8.1 Überforderung und Selbstunsicherheit). Auch die Mitarbeiter, die schon vor Initiierung des KPZs eine Weiterbildung in Palliative Care gemacht hatten, taten dies häufig, weil sie sich mit etwas Unbekanntem (sterbenden Patienten) konfrontiert sahen und Mittel und Wege lernen wollten, die dadurch entstehende Unsicherheit zu bekämpfen. In der Ausbildung von Ärzten und Pflegepersonal nimmt die Versorgung sterbender Patienten bisher wenig Raum ein. Hier besteht noch Verbesserungsbedarf (Jors et al. 2016). Die Göttinger Mitarbeiter beklagten eine mangelnde fachliche Vorbereitung auf das Thema Palliativmedizin vor Eröffnung des KPZs und wünschten sich Fortbildungen.

\subsection{Bedeutung der kollegialen Gemeinschaft}

Eine weitere große Handlungsstrategie gegen Überforderung und den Umgang mit neuen Herausforderungen zeigte sich, dass viele Mitarbeiter Unterstützung bei ihren Kollegen suchten. Zahlreiche Mitarbeiter schienen Halt in der kollegialen Gemeinschaft zu finden. Diese Handlungsstrategie wird auch in der Literatur deutlich (Spencer 1994). Damit diese Strategie weiterhin funktionieren kann, ist es wichtig, dass die intra- und interprofessionellen Konflikte nicht weiter zunehmen. Solche Schwierigkeiten der Zusammenarbeit finden sich auch in der Literatur und belasten bzw. komplizieren das gemeinsame Arbeiten der einzelnen Professionen (Lancaster et al. 2015; Liberati et al. 2016). Ursächlich ist häufig mangelnde bzw. fehlerhafte Kommunikation. Besonders zwischen Ärzteschaft und Pflegepersonal kommt es immer wieder zu Konflikten. In den Interviews wurde deutlich, dass die Teamarbeit insgesamt verbesserungswürdig ist. Das Pflegepersonal äußerte fast einstimmig Kritik am Verhalten der Ärzte. Von den Göttinger Medizinern wurden derartige interprofessionelle Uneinigkeiten nicht thematisiert. Dieses 
Ergebnis deckt sich mit den Ergebnissen einer amerikanischen Studie. Dort nahmen Ärzte und Pflegepersonal einer Intensivstation die interprofessionelle Zusammenarbeit auch sehr unterschiedlich wahr. Nur ein Drittel des Pflegepersonals bewertete die Zusammenarbeit und Kommunikation mit den Ärzten als gut bis sehr gut, wohingegen bei den Ärzten über $70 \%$ so urteilten (Thomas et al. 2003). Auch andere Studien identifizierten viele Mitarbeiterkonflikte in Krankenhäusern (Fassier und Azoulay 2010; Forbat et al. 2016). Forbat et al. (2016) stellten in ihrer Studie Kommunikationsprobleme, Uneinigkeiten bei der Behandlung und unrealistische Erwartungen als häufigste Ursachen für Auseinandersetzungen fest. Aber auch logistische und organisatorische Probleme konnten Konflikte verursachen. Besonders ausgeprägt waren Konflikte in der pädiatrischen Neurologie, weil das Personal dort mit schlechten Prognosen, großer Unsicherheit und komplexen multiprofessionellen Anforderungen zu kämpfen hat. Ähnliche Ursachen für die Entstehung von Auseinandersetzungen konnten auch Fassier und Azoulay (2010) beobachten. Diese Untersuchungen decken sich mit den Ergebnissen aus Göttingen. Auf der Neuropädiatrie bestanden einige Konflikte schon vor Entstehung des KPZs. Das erbaute KPZ führte jedoch durch die bewusste Konfrontation mit Kinderpalliativpatienten und die beschriebenen organisatorischen Schwierigkeiten während seiner Entstehung zu einer scheinbaren Zunahme der Mitarbeiterkonflikte.

\subsection{Stärken und Schwächen der Arbeit}

\subsubsection{Interviewsituation}

Zu Beginn der Studie wurde eine Interviewzeit von ca. 60 Minuten veranschlagt. Diese musste im Studienverlauf reduziert werden, da viele Interviewpartner einen Gesprächstermin während ihrer Arbeitszeit wünschten und keine 60 Minuten von ihrer Arbeit fernbleiben konnten (vgl. Kapitel 4.3.1 Qualitative Interviews). Die Interviewsituation während der Arbeitszeit war z. T. durch Unruhe und Zeitdruck geprägt. Telefonanrufe, Pieper, Kollegen u. ä. unterbrachen die Gesprächssituation, limitierten das Interview und stoppten teilweise einen narrativen Erzählfluss. Doch auch die Doktorandin selbst bemerkte, dass durch das vorgegebene Zeitfenster manchmal interessante Gesprächsinhalte nicht weiterverfolgt wurden, weil - z. B. bei fortgeschrittener Zeit - das Gespräch auf andere Themenkomplexe des Leitfadens gelenkt wurde. Eine Befragung außerhalb der Arbeitszeit wäre für die Interviewsituation teilweise förderlich gewesen, vielleicht auch ein Interviewort abseits des Stationsgeschehens. Die Interviews, die in der Mildred-Scheel-Akademie stattfanden, wurden z. B. nicht durch Kollegen, Anrufe oder 
ähnliches unterbrochen. Da die Interviewpartner allerdings hauptsächlich ein Interview während oder unmittelbar vor oder nach ihrer Arbeitszeit und an ihrem Arbeitsplatz wünschten, musste versucht werden, die limitierte Zeit optimal auszunutzen.

\subsubsection{Fehlendes Theoretisches Sampling}

Wie in Kapitel 4.4.4 Theoretisches Sampling bereits angeführt, fordern Glaser und Strauss (2010) für den Datenerhebungsprozess die Anwendung des Theoretischen Samplings. Verhindert Zeitmangel die gleichzeitige Datenerhebung, Kodierung und Analyse kann das Theoretische Sampling mit zuvor erhobenen Daten verwirklicht werden. Für dieses Vorgehen müssen - nach Glaser und Strauss - viele Daten verfügbar sein, um trotz allem eine Theorie von nötiger Dichte generieren zu können. Da sich nur 17 Mitarbeiter zur Teilnahme an der Studie bereit erklärten, konnte dieses Vorgehen nicht gewählt werden, da die Menge der verfügbaren Daten nicht ausreichend war, um einen Auswahlprozess durchzuführen. Es wurden deshalb alle durchgeführten Interviews ausgewertet und ein Theoretisches Sampling fand nicht statt. Einzelne Eigenschaften bestimmter Kategorien hätten mit zusätzlichen Daten noch weiter ausdifferenziert werden können. Trotz dieser ohne Zweifel bestehenden Limitation - sei angemerkt, dass die dargestellten Kategorien und ihre Eigenschaften gut ausgearbeitet werden und eine Theorie von einiger Dichte generiert werden konnte.

Außerdem war eine Generalisierung für einzelne Berufsgruppe nicht möglich, da teilweise zu wenige Vertreter einzelner Gruppen an der Studie teilnahmen.

\subsubsection{Besondere Stärken der Arbeit}

Hervorzuheben sei der Einblick in die komplexe Mitarbeiterperspektive, den diese Studie liefern konnte. Das qualitative Studiendesign ermöglichte eine sehr genaue Darstellung des Implementierungsprozesses aus Mitarbeitersicht, deutlich detaillierter und differenzierter, als es mit einem Fragebogen mit vorgefertigten Antworten der Fall gewesen wäre. Die Durchführung von narrativen Interviews ermöglichte eine präzise Darstellung der Meinungen und Erwartungen der Mitarbeiter. Die Interviewpartner konnten eigene narrative Schwerpunkte setzen, sodass Aspekte zur Sprache kamen, die im Vorhinein von der Arbeitsgruppe nicht erwartet worden waren, wie z. B. die organisatorischen Hindernisse. Auch Einblicke in Strukturen und Abläufe eines Krankenhauses konnten gegeben werden. Somit kann die Studie genutzt werden, um den Entstehungsprozess und die Anlaufphase kritisch zu beleuchten und konstruktiv an Verbesserungen zu arbeiten. 


\subsection{Fazit und Ausblick}

Die Untersuchung des Implementierungsprozesses des KPZs in Göttingen offenbarte Stärken und Schwächen: Die Ausstattungsunterschiede zwischen dem KPZ und den regulären Stationszimmern der Neuropädiatrie waren augenscheinlich und stellten - aus Sicht des Personals - eine mögliche Belastung für den ,Stationsfrieden“ dar. Die Ausstattung des KPZs ermöglicht eine verbesserte stationäre Versorgung für Kinder mit LBE und LLE, kann jedoch, so die Befürchtung mancher Mitarbeiter, bei den anderen Stationspatienten und deren Familien Neid auslösen. Ob sich diese Befürchtungen des Personals bestätigt haben, könnte nur durch eine Befragung von betroffenen Eltern erfahren werden. Das Auftauchen des Begriffes „Klassengesellschaft auf der Station“, was als Vorwand für eine Kritik an der gefühlten mangelnden Einbeziehung des Personals in den Planungs- und Entstehungsprozess des KPZs interpretiert werden kann (vgl. Kapitel 6.1.1 Die Antizipation von Neid), macht deutlich, dass eine Aufarbeitung dieses Aspekts des Implementierungsprozesses stattfinden sollte.

Viele Mitarbeiter befürchteten durch das KPZ eine Zunahme der Konfrontation mit sterbenden Kindern und eine daraus resultierende große emotionale Belastung. Der angenommene gesteigerte Kontakt mit versterbenden Patienten hat sich bei Nutzung des Zimmers bisher nicht bestätigt. In den ersten 18 Monaten Nutzung verstarb nur ein Kind im KPZ. Alle anderen Patienten verließen das Zimmer wieder und wurden bzw. werden ambulant versorgt. Die Konfrontation mit sterbenden Patienten ist somit nicht gestiegen. Unklar bleibt, inwieweit schon die Befürchtung einer verstärkten Konfrontation mit sterbenden Patienten die psychische Belastung der Mitarbeiter erhöht hat. Dies müsste in einer weiteren Mitarbeiterbefragung untersucht werden.

Organisatorische Schwierigkeiten erschwerten den Eingliederungsprozess des KPZs aus Sicht des Personals. Dabei wurde deutlich, wie wichtig eine Information über und eine Teilhabe des Personals an Veränderungen am Arbeitsplatz ist. An einem Arbeitsplatz wie einem Krankenhaus muss vielleicht besonders beachtet werden, dass z. B. aufgrund von Schichtplänen o. ä. nicht immer alle Mitarbeiter, für die die neuen Informationen wichtig sind, gleichzeitig vor Ort sind und so einzelne Mitarbeiter Informationen sehr spät, nicht vollständig oder gar nicht erhalten. Hier könnte es ratsam sein, Informationsveranstaltungen oder andere Formen der Informationsweitergabe (z. B. Flyer, Briefe) in regelmäßigen Abständen zu wiederholen. Das Gefühl des Personals über ausreichend Informationen zu verfügen, könnte die Entstehung von Überforderung und Selbstunsicherheit verhindern. 
Bei der Integration eines solchen Zimmers auf einer Normalstation scheint eine Aufklärung über Aufgaben und Ziele von Kinderpalliativmedizin wichtig zu sein. Fachliche Weiterbildungen des Personals scheinen eine Voraussetzung dafür zu sein, dass das Personal sich sicher beim Umgang mit Palliativpatienten fühlt. Während der Anlaufphase des Zimmers machte es den Eindruck, dass eine Überforderung und Unsicherheit bei einigen Göttinger Mitarbeitern bestand. Zu bedenken ist an dieser Stelle, dass die Befragung mehr als 12 Monate zurückliegt. Um sicherzustellen, dass die Mitarbeiter nun über einen einheitlichen Wissensstand bezüglich Kinderpalliativmedizin verfügen und Berührungsängste abgebaut wurden, sollten eine nachträgliche Aufklärung über Kinderpalliativmedizin und fachliche Fortbildungen erfolgen. Besteht ein größeres Wissen über Aufgaben und Ziele von Palliativmedizin, so stellt die Berührung mit Kindern in der Palliativversorgung möglichweise eine weniger große Herausforderung dar.

Abgesehen von der Kritik an den beschriebenen Schwierigkeiten während des Entstehungsprozesses des KPZs, waren die meisten Mitarbeiter gegenüber einem Palliativzimmer für Kinder nicht abgeneigt und begrüßten ein solches Zimmer an ihrem Arbeitsplatz. Die Erweiterung des Aufgabenbereiches der Station im Allgemeinen und des eigenen Aufgabenbereiches im Speziellen wurde auch als Chance wahrgenommen. Der einzelne Mitarbeiter kann einen neuen beruflichen Schwerpunkt setzen, der eine Kompetenzerweiterung darstellt. 


\section{$7 \quad$ Zusammenfassung}

Fragestellung: Im Rahmen der Versorgungsstrukturerweiterung für Palliativkinder entstand ein Kinderpalliativzimmer (KPZ) an der UMG. Die Implementierung des KPZs erfolgte auf einer Station mit kurativer Ausrichtung. Untersucht wurde, wie die Mitarbeiter diesen Implementierungsprozess erlebten und welche Erwartungen sie an das KPZ hatten.

Design und Methode: Es wurden offene Leitfadeninterviews mit Mitarbeitern verschiedener Berufe geführt ( $\mathrm{n}=17)$. Die Auswertung erfolgte mittels Grounded Theory (Glaser/Strauss; Strauss/Corbin) unter der Verwendung induktiver Schlussfolgerungen.

Ergebnisse: In der Konfrontation mit dem KPZ zeigte sich bei den Mitarbeitern als zentrales Phänomen das Gefühl einer „Klassengesellschaft“ auf der Station. Die vordergründige Begründung dieser Empfindung mit den signifikanten Ausstattungsunterschieden zwischen dem KPZ und den regulären Stationszimmern kann als Ausdruck für die Unzufriedenheit des Personals mit einzelnen Aspekten des Implementierungsprozesses verstanden werden. Ursächlich für die Missstimmung waren organisatorische Schwierigkeiten während der Entstehungsphase sowie eine Abgrenzungsproblematik des eigenen Aufgaben- und Verantwortungsbereiches bei den Mitarbeitern. Das Personal reagierte distanziert auf die gefühlte „Klassengesellschaft“. Beeinflusst wurde diese Reaktion durch eine Sorge vor verstärkter und psychisch belastender Konfrontation mit sterbenden Patienten. Zusätzlich fühlten sich viele der befragten Mitarbeiter in den Entstehungsprozess des Zimmers zu wenig integriert. Intra- und interprofessionelle Konflikte komplizierten die Zusammenarbeit im Team. Die Distanzierung hatte eine Resignation zur Folge, welche mit unklaren neuen Arbeitsanforderungen zu Überforderung führte und durch unsicheres Wissen über Aufgaben und Ziele von Kinderpalliativmedizin verstärkt wurde. Trotz dieser Schwierigkeiten während des Entstehungsprozesses sahen einige Mitarbeiter im KPZ die Chance zur eigenen Kompetenzerweiterung. Ein neuer beruflicher Schwerpunkt konnte gesetzt werden, der trotz aller Herausforderung als positive Ergänzung des Arbeitsalltages wahrgenommen wurde.

Schlussfolgerung: Um der Unzufriedenheit und Überforderung der Mitarbeiter entgegenzuwirken und eine stärkere Akzeptanz des KPZs zu erreichen und damit seinen Erfolg zu erhöhen, sollte eine stärkere Einbeziehung des Personals in das Projekt KPZ erfolgen. Zusätzlich scheint eine Aufklärung bezüglich Kinderpalliativmedizin und eine fachliche Weiterbildung empfehlenswert zu sein. 


\section{Anhang}

Georg-August-Universität Göttingen

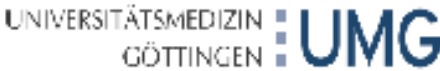
Klinik für Palliativmedizin • Forschungsbereich

Von-Siebold-Str.3 • 37075 Göttingen

Direktor: Prof. Friedemann Nauck

Wiss. Studienleitung: Dr. Gabriella Marx

\section{- Information und Aufklärung für Mitarbeiterlnnen •}

Teilnahme an einem Interview

im Rahmen der Studie zur Befragung von Mitarbeitern der Abteilungen Neuropädiatrie und Pädiatrische Hämatologie und Onkologie, bezüglich des neu entstehenden Kinderpalliativzimmers

Sehr geehrte Dame, sehr geehrter Herr,

im Rahmen der oben genannten Studie wird in der Klinik für Palliativmedizin untersucht, inwiefern durch dieses neue Modell eines Kinderpalliativzimmers, integriert auf einer Kinderstation, die bestehenden Versorgungsstrukturen für schwerstkranke Kinder erweitert werden.

Aus diesem Anlass möchte ich gerne ein persönliches Gespräch (Interview) mit Ihnen führen, um mehr über die Perspektive und Erfahrungen der MitarbeiterInnen der Stationen Neuropädiatrie und Pādiatrische Hāmatologie und Onkologie zu erfahren. Das Interview findet an einem Ort und zu einer Zeit Ihrer Wahl statt. Mich interessiert wie Sie den Arbeitsalltag auf Ihrer Station erleben. Ein Interview dauert ca. eine Stunde und wird von mir persönlich durchgeführt. Sie haben während des Interviews jederzeit die Möglichkeit, dieses zu unterbrechen oder abzubrechen. Im Anschluss an das Interview möchte ich Sie bitten, einen begleitenden Kurzfragebogen mit Daten zu Ihrer Person auszufüllen.

Zur besseren Auswertung werden die Interviews auf Tonband aufgezeichnet und wörtlich niedergeschrieben. Nach Beendigung der Studie werden die Originalaufnahmen zwar archiviert, aber nicht für weitere Studien oder im Unterricht für die Studierenden verwendet. Die Auswertungen der Interviews erfolgen pseudonymisiert, d.h. ohne Verwendung Ihres Namens, so dass keine Rückschlüsse auf Ihre Person oder Angehörigen möglich sein werden. Eine Weitergabe dieser Informationen an Dritte (z.B. an den behandelnden Arzt, die Pflegedienstleitung oder lhre Angehörigen) ist ausgeschlossen.

Die Teilnahme an dieser Studie ist freiwilliq! Aus Gründen der Qualitätssicherung werden Ihre Daten sowie die Tonbandaufnahmen für einen Zeitraum von 10 Jahren unter den Bedingungen des Datenschutzes gespeichert. Sie können jederzeit ohne Angabe von Grūnden Ihre Teilnahme beenden, ohne dass Ihnen irgendwelche Nachteile entstehen. In diesem Fall würden alle bis dahin von Ihnen erhobenen Daten von mir vernichtet werden.

Die Ergebnisse der Studie dienen ausschließlich wissenschaftlichen Zwecken und werden in Fachzeitschriften veröffentlicht. Ich informiere Sie gerne über die Ergebnisse, wenn Sie dies wünschen.

Ich würde mich sehr freuen, wenn Sie teilnehmen. Ich kontaktiere Sie in den nächsten Tagen, um Ihre Teilnahme an der Studie zu erfragen und um ggf. einen Interviewtermin zu vereinbaren.

Wenn Sie Fragen haben, kōnnen Sie sich gerne bei mir melden.

Anhang A Informationsschreiben 


\section{EINWILLIGUNGSERKLÄRUNG}

zur Teilnahme an einem Interview

Studie zur Befragung von Mitarbeitern und Eltern der Stationen Neuropädiatrie und Pädiatrische Hämatologie und Onkologie bezüglich des neu entstehenden Kinderpalliativzimmers

\section{Nachname, Vorname:}

Anschrift:

Ich habe die Teilnehmerinformation und diese Einwilligungserklärung gelesen und in Ruhe durchdacht. Art, Zweck und Dauer der Studie wurden mir erläutert, und ich wurde darüber informiert, was von mir erwartet wird.

Ich hatte Gelegenheit, Fragen zu stellen und alle meine Fragen wurden zu meiner Zufriedenheit beantwortet. Ich habe mich nach ausreichend Bedenkzeit zur freiwilligen Teilnahme an den Interviews mit begleitendem Kurzfragebogen entschieden. Ich weiß, dass die Teilnahme mit keinerlei persönlichem Nutzen oder mit Nachteilen für mich verbunden ist.

Ich weiß, dass ich jederzeit diese Zustimmung ohne Angabe von Gründen zurückziehen kann, ohne dass mir daraus Nachteile entstehen. Die bisher erhobenen Daten würden in diesem Fall vernichtet werden.

Ich erlaube ausdrücklich, dass durch die an dieser Studie beteiligten Mitarbeiter meine Angaben während des Interviews audiodigital aufgezeichnet werden.

Ich bin damit einverstanden, dass die im Rahmen der Studie erhobenen Daten für 10 Jahre in pseudonymisierter Form und unter den Bedingungen des Datenschutzes gespeichert und zu Forschungszwecken verwendet werden. Bei Widerruf der Einwilligung werden alle bis dahin von mir erhobenen Daten vernichtet.

Mir ist bekannt, dass die Ergebnisse der Studie später in Fachzeitschriften veröffentlich werden. Die personenbezogenen Daten werden nicht an Dritte weitergegeben.

Ich habe die Informationen zur Studie in schriftlicher Form und eine Kopie der Einverständniserklärung für meine Unterlagen erhalten und erkläre hiermit meine freiwillige Teilnahme an dem Interview.

Ort, Datum

Unterschrift Teilnehmer
Ort, Datum

Unterschrift Studienleitung

Anhang B Intervieweinwilligung 
Georg-August-Universität Göttingen

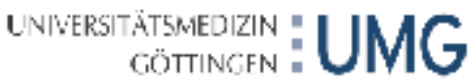

Klinik für Palliativmedizin • Forschungsbereich

Von-Siebold-Str.3 • 37075 Göttingen

Direktor: Prof. Friedemann Nauck

Wiss. Studienleitung: Dr. Gabriella Marx

\title{
„Studie zur Befragung von Mitarbeiterinnen und Mitarbeitern der Abteilungen Neuropädiatrie und
}

Pädiatrische Hämatologie und Onkologie bezüglich des

neu entstehenden Kinderpalliativzimmers auf der Station Neuropädiatrie"

Kurzfragebogen für Mitarbeiterinnen

\begin{abstract}
Liebe Teilnehmerin,
lieber Teilnehmer,

Sie haben sich bereit erklärt, an einem Interview im Rahmen der o.g. Studie teilzunehmen. Dieser Fragebogen ist eine Vorbereitung auf das Interview, das ich gerne mit Ihnen führen möchte.

Wenn Sie eine Frage nicht beantworten können oder unsicher bei der Beantwortung sind, lassen Sie das Feld einfach aus.
\end{abstract}

Anhang C Kurzfragebogen 


\section{Personenbezogene Fragen}

Welches Geschlecht haben Sie?

D Weiblich a Männlich

Wie alt sind Sie?

Ich bin Jahre alt.

Welchen Familienstand haben Sie?

- In einer Partnerschaft

lebend

D Nicht in einer Partnerschaft

lebend

D Keine Angabe

Haben Sie Kinder?

- Ja ich habe Kind/Kinder

- Nein ich habe keine Kinder

Welchen höchsten allgemeinbildenden Schulabschluss haben Sie?

- Hauptschulabschluss

- Realschulabschluss

- Polytechnische Oberschule

a Fachhochschulreife

- Allgemeine oder fachgebundene Hochschulreife (Abitur bzw. EOS)

- keinen Schulabschluss

- Andere:

Welchen beruflichen Ausbildungsabschluss haben Sie? (Mehrfachnennungen möglich)

- Beruflich-betrieblich (Lehre)

D Beruflich-schulisch (Berufsfach-. Handelsschule)

D Hochschule

a Fach-, Meister-, Technikerschule, Berufsoder Fachakademie

口 Weiß nicht

a Fachhochschule

a Andere:

Welchen Beruf üben Sie aus?

- Arztin/Arzt, Fachrichtung: Zusatzweiterbildung:

a Pflegerin/Pfleger, Fachrichtung: Zusatzweiterbildung:

D Psychologin/Psychologe

- Sozialarbeiterin/Sozialarbeiter / Sozialpädagogin/Sozialpädagoge

D Physiotherapeutin/Physiotherapeut

- Andere:

Wie lange üben Sie Ihren derzeitigen Beruf schon aus?

Seit: Jahren.

Sind Sie gläubig/spirituell?

口 Ja Dein a Weiß nicht 
Üben Sie aktiv eine Religion aus?
- Ja
a Nein
ㅁ Weiß nicht
- Keine Angabe

Wenn ja, welcher Glaubensrichtung fühlen Sie sich zugehörig?
- Keine Angabe
- Hinduismus
- Buddhismus
口 Islam
a Christentum - evangelisch
口 Judentum
- Christentum - katholisch
a Andere:

Wie lange arbeiten Sie schon auf der Station Neuropädiatrie der UMG?

Ich arbeite seit Jahren auf dieser Station.

Wussten Sie schon vor unserem Interview von dem neuen Kinderpalliativzimmer?

口 Ja

口 Nein

D Weiß nicht

Wenn ja, wie haben Sie davon erfahren?

口 Informationsplakat $\mathbf{a}$ Information auf der Station

口 durch andere Personen

a Andere: 


\section{Transkriptionsregeln}

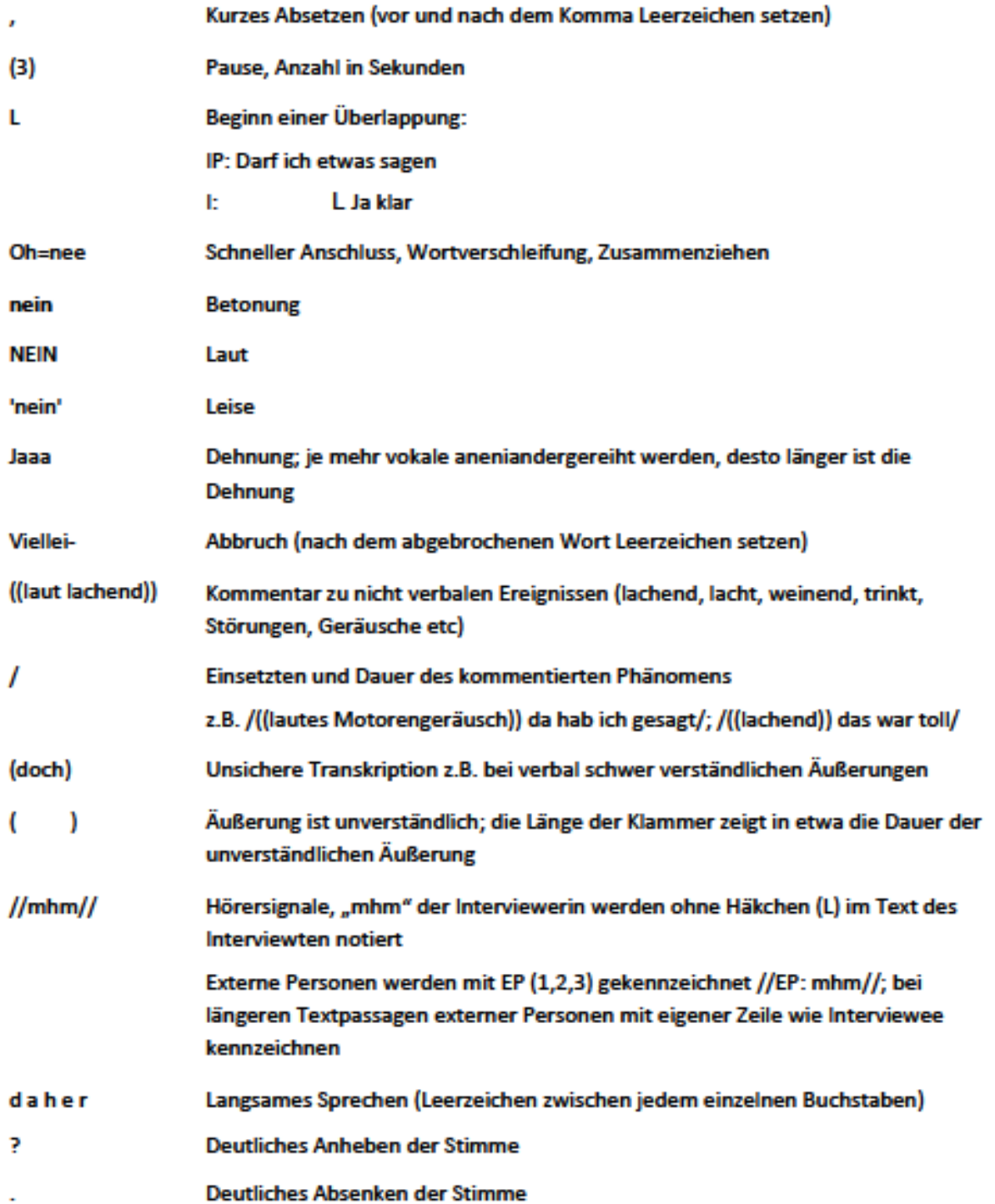

Anhang C Transkriptionsregeln 


\section{$9 \quad$ Literaturverzeichnis}

ACT: Association for Children with Life-threatening or Terminal Conditions and their Families (ACT) and the Royal College of Paediatrics and Child Health (RCPCH): A Guide to the Development of Children's Palliative Care Services. 3. Auflage; Bristol, UK 2009

Bohnsack R, Marotzki W, Meuser M: Hauptbegriffe qualitative Sozialforschung: ein Wörterbuch. Leske+Budrich, Opladen 2003

Booth RZ (2002): The nursing shortage: a worldwide problem. Rev Lat Am Enfermagem $\underline{10}, 392-400$

Borasino S, Morrison W, Silberman J, Nelson RM, Feudtner C (2008): Physicians' contact with families after the death of pediatric patients: a survey of pediatric critical care practitioners' beliefs and self-reported practices. Pediatrics $\underline{122}$, e1174-1178

Brent DA (1983): A death in the family: the pediatrician's role. Pediatrics $\underline{72}, 645-651$ Breyer J, Kunin H, Kalish LA, Patenaude AF (1993): The adjustment of siblings of pediatric cancer patients—a sibling and parent perspective. Psychooncology $\underline{2}, 201-208$ Bright KL, Huff MB, Hollon K (2009): A Broken Heart—The Physician's Role: Bereaved Parents' Perceptions of Interactions with Physicians. Clin Pediatr (Phila) 48, 376-382 Brosius H-B, Koschel F, Haas A: Methoden der empirischen Kommunikationsforschung: eine Einführung. (Studienbücher zur Kommunikations- und Medienwissenschaft). 4. überarb. und erw. Auflage; VS Verlag für Sozialwissenschaften, Wiesbaden 2008 Bucher A: Geiz, Trägheit, Neid \& Co. in Therapie und Seelsorge: Psychologie der 7 Todsünden. Springer-Verlag, Berlin 2012

Bürgin D: Das Kind, die lebensbedrohende Krankheit und der Tod. 1. Auflage; Huber, Bern 1978

Cahn EN: The sense of injustice. Indiana University Press, Bloomington 1964 Cain AC, Fast I, Erickson ME (1964): Children's disturbed reactions to the death of a sibling. Am J Orthopsychiatry $\underline{34}, 741-752$

Carter BS, Howenstein M, Gilmer MJ, Throop P, France D, Whitlock JA (2004): Circumstances Surrounding the Deaths of Hospitalized Children: Opportunities for Pediatric Palliative Care. Pediatrics 114, e361-e366 
Centeno C, Clark D, Lynch T, Racafort J, Praill D, De Lima L, Greenwood A, Flores LA, Brasch S, Giordano A, EAPC Task Force (2007): Facts and indicators on palliative care development in 52 countries of the WHO European region: results of an EAPC Task Force. Palliat Med 21, 463-471

Contro NA, Larson J, Scofield S, Sourkes B, Cohen HJ (2004): Hospital staff and family perspectives regarding quality of pediatric palliative care. Pediatrics $\underline{114}, 1248-1252$

Davies B, Clarke D, Connaughty S, Cook K, MacKenzie B, McCormick J, O’Loane M, Stutzer C (1996): Caring for dying children: nurses' experiences. Pediatr Nurs $\underline{22}, 500-507$

Dominica F (1987): Reflections on death in childhood. Br Med J Clin Res Ed 294, 108-110

Dussel V, Kreicbergs U, Hilden JM, Watterson J, Moore C, Turner BG, Weeks JC, Wolfe J (2009): Looking beyond where children die: determinants and effects of planning a child's location of death. J Pain Symptom Manage 37, 33-43

Fassier T, Azoulay E (2010): Conflicts and communication gaps in the intensive care unit. Curr Opin Crit Care 16, 654-665

Feudtner C, Hays RM, Haynes G, Geyer JR, Neff JM, Koepsell TD (2001): Deaths attributed to pediatric complex chronic conditions: national trends and implications for supportive care services. Pediatrics $\underline{107}$, E99

Feudtner C, Christakis DA, Zimmerman FJ, Muldoon JH, Neff JM, Koepsell TD (2002): Characteristics of deaths occurring in children's hospitals: implications for supportive care services. Pediatrics $\underline{109}, 887-893$

Feudtner C, Feinstein JA, Satchell M, Zhao H, Kang TI (2007): Shifting place of death among children with complex chronic conditions in the United States, 1989-2003. JAMA $\underline{297}, 2725-2732$

Flick U, Kardorff E, Steinke I: Qualitative Forschung: ein Handbuch. (Rororo Taschenbücher 55628). 10. Auflage; Rowohlt-Taschenbuch-Verlag, Reinbek bei Hamburg 2013

Forbat L, Sayer C, McNamee P, Menson E, Barclay S (2016): Conflict in a paediatric hospital: a prospective mixed-method study. Arch Dis Child 101, 23-27

Foster GM (1972): The anatomy of envy: a study in symbolic behavior. Curr Anthropol $\underline{13}$, $165-202$

Führer M (2011): Kinderpalliativmedizin. Monatsschr Kinderheilkd 159, 583-596 
Garten L: Grundlagen neonatologischer Palliativversorgung. In: Garten L, Hude K von der (Hrsg.): Palliativversorgung und Trauerbegleitung in der Neonatologie. Springer-Verlag, Heidelberg 2014, 3-18

Glaser BG, Strauss AL: Grounded theory: Strategien qualitativer Forschung. (Gesundheitswissenschaften : MethodenProgrammbereich Gesundheit). 3. unveränd. Auflage; Huber, Bern 2010

Goodenough B, Drew D, Higgins S, Trethewie S (2004): Bereavement outcomes for parents who lose a child to cancer: are place of death and sex of parent associated with differences in psychological functioning? Psychooncology $\underline{13}, 779-791$

Graham J, Ramirez AJ, Cull A, Finlay I, Hoy A, Richards MA (1996): Job stress and satisfaction among palliative physicians. Palliat Med $\underline{10}, 185-194$

Granek L, Bartels U, Scheinemann K, Labrecque M, Barrera M (2015): Grief reactions and impact of patient death on pediatric oncologists. Pediatr Blood Cancer $\underline{62}, 134-142$

Hassmiller SB, Cozine M (2006): Addressing the nurse shortage to improve the quality of patient care. Health Aff Proj Hope 25, 268-274

Hemsley-Brown J, Foskett NH (1999): Career desirability: young people's perceptions of nursing as a career. J Adv Nurs $\underline{29}, 1342-1350$

Heponiemi T, Puttonen S, Elovainio M (2014): On-call work and physicians' well-being: Testing the potential mediators. Occup Med $\underline{64}, 352-357$

Holland JC (2002): Management of grief and loss: medicine's obligation and challenge. J Am Med Womens Assoc 1972 57, 95-96

Humburg S: Mitarbeitermotivation im Krankenhaus: das Organisationsklima und seine Bedeutung für das Qualitätsmanagement. (Schriftenreihe zur angewandten Sozialpsychologie.). Dt. Inst.-Verlag, Köln 2001

Husebø S: Kommunikation. In: Husebø S, Klaschik E (Hrsg.): Palliativmedizin Grundlagen und Praxis. 5. Auflage; Springer-Verlag, Heidelberg 2009, 147-201 James L, Johnson B (1997): The needs of parents of pediatric oncology patients during the palliative care phase. J Pediatr Oncol Nurs Off J Assoc Pediatr Oncol Nurses 14, 83-95 Janiszewski Goodin H (2003): The nursing shortage in the United States of America: an integrative review of the literature. J Adv Nurs $\underline{43}, 335-343$ 
Jens W, Küng H: Menschenwürdig sterben: ein Plädoyer für Selbstverantwortung. 2.

Auflage; Piper, München [u.a.] 1995

Jors K, Seibel K, Bardenheuer H, Buchheidt D, Mayer-Steinacker R, Viehrig M, Xander C, Becker G (2016): Education in End-of-Life Care: What Do Experienced Professionals Find Important? J Cancer Educ Off J Am Assoc Cancer Educ 31, 272-278

Kassam A, Skiadaresis J, Alexander S, Wolfe J (2014): Parent and clinician preferences for location of end-of-life care: Home, hospital or freestanding hospice? Pediatr Blood Cancer $\underline{61}, 859-864$

Korte H: Einführung in Hauptbegriffe der Soziologie. 5. erw. u. aktual. Auflage; Leske+ Budrich, Opladen 2000

Krauss H: Kleines Lexikon der Engel: von Ariel bis Zebaoth. 2. Auflage; Beck, München 2002

Kreicbergs U, Valdimarsdóttir U, Onelöv E, Henter J-I, Steineck G (2004): Talking about death with children who have severe malignant disease. N Engl J Med 351, 1175-1186

Lancaster G, Kolakowsky-Hayner S, Kovacich J, Greer-Williams N (2015):

Interdisciplinary communication and collaboration among physicians, nurses, and unlicensed assistive personnel. J Nurs Scholarsh $\underline{47}, 275-284$

Lantos JD, Berger AC, Zucker AR (1993): Do-not-resuscitate orders in a children's hospital. Crit Care Med 21, 52-55

Lauterbach K: Der Zweiklassenstaat: wie die Privilegierten Deutschland ruinieren. Rowohlt Taschenbuch Verlag, Reinbek bei Hamburg 2008

Leuzinger A, Luterbacher T: Mitarbeiterführung im Krankenhaus: Spital, Klinik und Heim. (Management im Gesundheitswesen). 3. unveränd. Auflage; Huber, Bern 2000

Liberati EG, Gorli M, Scaratti G (2016): Invisible walls within multidisciplinary teams: Disciplinary boundaries and their effects on integrated care. Soc Sci Med 1982 150, 31-39

McCallum DE, Byrne P, Bruera E (2000): How children die in hospital. J Pain Symptom Manage 20, 417-423

Morrison W, Berkowitz I (2007): Do not attempt resuscitation orders in pediatrics. Pediatr Clin North Am $\underline{54}, 757-771$

Mulhern RK, Lauer ME, Hoffmann RG (1983): Death of a child at home or in the hospital: subsequent psychological adjustment of the family. Pediatrics $\underline{71}, 743-747$ 
Müller-Dofel M: Interviews führen: Ein Handbuch für Ausbildung und Praxis.

(Journalistische Praxis); Springer Fachmedien, Wiesbaden 2013

Murray JS (1999): Siblings of children with cancer: a review of the literature. J Pediatr

Oncol Nurs Off J Assoc Pediatr Oncol Nurses 16, 25-34

Nauck F, Gärtner J (2015): Palliativmedizin bei Erwachsenen, Kindern und Jugendlichen -

Einführung in das Thema. Kinderärztl Prax $\underline{86}$, 78-82

Nolte-Buchholtz DS, Janisch M (2015): Pädiatrische Palliativversorgung. Monatsschr Kinderheilkd 163, 1037-1049

Parrott WG, Smith RH (1993): Distinguishing the experiences of envy and jealousy. J Pers Soc Psychol 64, 906-920

Radbruch L, Payne S (2011): Standarts und Richtlinien für Hospiz- und Palliativversorgung in Europa: Teil 1 Weißbuch zu Empfehlungen der Europäischen Gesellschaft für Palliative Care (EAPC). Z Palliativmed 12, 216-227

Radbruch L, Nauck F, Sabatowski R: Wegweiser Hospiz und Palliativmedizin Deutschland 2005. Hospiz Verlag, Esslingen 2005

Rando TA (1985): Bereaved parents: particular difficulties, unique factors, and treatment issues. Soc Work $\underline{30}, 19-23$

Rellensmann DG, Hasan C (2009): Empfehlungen zum Vorgehen in Notfallsituationen. Monatsschr Kinderheilkd 157, 38-42

Rickheit G, Herrmann T, Deutsch W, Ungeheuer G, Burkhardt A (Hrsg.):

Psycholinguistik: ein internationales Handbuch. de Gruyter, Berlin [u.a.] 2003

Rosenthal G: Interpretative Sozialforschung: eine Einführung. (Grundlagentexte Soziologie). 5., aktualisierte und ergänzte Auflage; Beltz Juventa, Weinheim 2015

Sahler OJ, Frager G, Levetown M, Cohn FG, Lipson MA (2000): Medical education about end-of-life care in the pediatric setting: principles, challenges, and opportunities. Pediatrics $\underline{105}, 575-584$

Schmalenberg C, Kramer M (2009): Nurse-physician relationships in hospitals: 20,000 nurses tell their story. Crit Care Nurse 29, 74-83

Schnell R: Methoden der empirischen Sozialforschung. 10. überarbeitete Auflage; Oldenbourg Verlag, München 2013 
Schulze Ehring F, Weber C: „Zwei-Klassen-Medizin“: Zur Diskussion von Leistungs- und Qualitätsunterschieden im deutschen Gesundheitswesen. In: v. Bandelow NC, Eckert F, Rüsenberg R. (Hrsg.): Gesundheit 2030 Qualitätsorientierung im Fokus von Politik, Wirtschaft, Selbstverwaltung und Wissenschaft, 1. Auflage; VS Verlag für Sozialwissenschaften, Wiesbaden 2009, 135-149

Siden H, Miller M, Straatman L, Omesi L, Tucker T, Collins JJ (2008): A report on location of death in paediatric palliative care between home, hospice and hospital. Palliat Med $\underline{22}$, $831-834$

Smith RH (Hrsg.): Envy: theory and research. (Series in affective science). Oxford University Press, Oxford 2008

Smith RH, Turner TJ, Garonzik R, Leach CW, Urch-Druskat V, Weston CM (1996): Envy and Schadenfreude. Pers Soc Psychol Bull 22, 158-168

Spencer L (1994): How do nurses deal with their own grief when a patient dies on an intensive care unit, and what help can be given to enable them to overcome their grief effectively? J Adv Nurs $\underline{19}, 1141-1150$

Strauss AL, Corbin JM: Grounded theory: Grundlagen qualitativer Sozialforschung. Unveränd. Nachdr. der letzten Auflage, 1996; Beltz, Weinheim 1999

Strübing J: Grounded Theory: zur sozialtheoretischen und epistemologischen Fundierung des Verfahrens der empirisch begründeten Theoriebildung. (Qualitative Sozialforschung 15). 2. überarb. und erw. Auflage; VS Verlag für Sozialwissenschaften, Wiesbaden 2008 Surkan PJ, Kreicbergs U, Valdimarsdóttir U, Nyberg U, Onelöv E, Dickman PW, Steineck G (2006): Perceptions of inadequate health care and feelings of guilt in parents after the death of a child to a malignancy: a population-based long-term follow-up. J Palliat Med $\underline{9}$, $317-331$

Theunissen JM, Hoogerbrugge PM, van Achterberg T, Prins JB, Vernooij-Dassen MJ, van den Ende CH (2007): Symptoms in the palliative phase of children with cancer. Pediatr Blood Cancer 49, 160-165

Thomas EJ, Sexton JB, Helmreich RL (2003): Discrepant attitudes about teamwork among critical care nurses and physicians. Crit Care Med $\underline{31}$, 956-959

Vickers J, Thompson A, Collins GS, Childs M, Hain R, Paediatric Oncology Nurses' Forum/United Kingdom Children's Cancer Study Group Palliative Care Working Group (2007): Place and provision of palliative care for children with progressive cancer: a study 
by the Paediatric Oncology Nurses' Forum/United Kingdom Children's Cancer Study Group Palliative Care Working Group. J Clin Oncol 25, 4472-4476

Vos MA de, Bos AP, Plötz FB, Heerde M van, Graaff BM de, Tates K, Truog RD, Willems DL (2015): Talking With Parents About End-of-Life Decisions for Their Children.

Pediatrics $\underline{135}$, e465-e476

Whippen DA, Canellos GP (1991): Burnout syndrome in the practice of oncology: results of a random survey of 1,000 oncologists. J Clin Oncol Off J Am Soc Clin Oncol ㄹ, 19161920

White KR, Coyne PJ, Patel UB (2001): Are nurses adequately prepared for end-of-life care? J Nurs Scholarsh $\underline{33}, 147-151$

Williams PD (1997): Siblings and pediatric chronic illness: a review of the literature. Int J Nurs Stud $\underline{34}, 312-323$

Wolfe J, Grier HE, Klar N, Levin SB, Ellenbogen JM, Salem-Schatz S, Emanuel EJ, Weeks JC (2000): Symptoms and suffering at the end of life in children with cancer. N Engl J Med $\underline{342}, 326-333$

Zernikow B, Nauck F (2008): Pädiatrische Palliativmedizin: Kindern ein „gutes Sterben“ ermöglichen. Dtsch Ärztebl 105, 1376-1380

Zernikow B, Henkel W: Palliativversorgung von Kindern und Jugendlichen. In: S. Husebø, E. Klaschik (Hrsg.): Palliativmedizin Grundlagen und Praxis. 5. Auflage; Springer-Verlag, Heidelberg 2009, 436-446

Akademie Kinder-Hospiz Sternenbrücke. https://sternenbruecke.de/upload/dede/download/Broschuere_Akademie\%202016_Nachdruck-GO_web.pdf; Zugriff am 18.07.2016

Datenerhebung Deutscher Hospiz- u. PalliativVerband. http://www.dhpv.de/tl_files/public/Service/statistik/20160411_Bericht_StatHospizePalli ativ.pdf; Zugriff am 19.04.2016

Flyer Kinderpalliativmedizin. http://www.childrenms.de/redirect_hosts/sappv/downloads/Kinderpalliativmedizin_flyer.pdf; Zugriff am 30.08 .2016

Jahresbericht 2015 - Deutsches Kinderkrebsregister. http://www.kinderkrebsregister.de/typo3temp/secure_downloads/29942/0/f474d4594c6b 5a8805c4e629db249872e05d69ddb/jb2015_s.pdf; Zugriff am 03.04.2016 
Kinderpalliativzentrum Datteln. https://www.kinderpalliativzentrum.de/de/stationlichtblicke; Zugriff am 09.11.2016

Kinderpalliativzentrum Göttingen. http://www.kinderpalliativmedizin-goettingen.de/; Zugriff am 14.05.2016

Statistisches Bundesamt Todesursachen in Deutschland 2014

https://www.destatis.de/DE/Publikationen/Thematisch/Gesundheit/Todesursachen/To desursachen2120400147004.pdf?_blob=publicationFile; Zugriff am 03.04.2016

WHO Definition of Palliative Care. http://www.who.int/cancer/palliative/definition/en/; Zugriff am 10.06.2016 


\section{Danksagung}

Prof. Dr. Friedemann Nauck möchte ich für die Überlassung des hochinteressanten Themas und die stets freundliche und hilfsbereite Unterstützung bei meiner Arbeit danken. Mein besonderer Dank gilt Frau Dr. Gabriella Marx für die außerordentlich gute Betreuung. Sie lieferte mir wertvolle Förderung bei der Planung und Durchführung der Interviews und der Auswertung meiner Daten.

Bedanken möchte ich mich auch bei Sonja Owusu-Boakje, die mir bei Fragen und Problemen zur Seite stand und mich während der gesamten Zeit konstruktiv unterstützte.

Mein Dank gilt außerdem allen Mitarbeitern des Forschungsbereiches der Klinik für Palliativmedizin in Göttingen, die mir einen Arbeitsplatz zur Verfügung stellten. Ich wurde sehr freundlich aufgenommen, und man stand mir stets mit Rat und Tat zur Seite. Die initiierten Forschungswerkstätten für qualitative Forschung in der Palliativmedizin halfen mir stets, meine Arbeit weiter voranzubringen. 


\section{Lebenslauf}

Mein Name ist Janna Bardt, geb. Flach, und ich wurde am 11.03.1990 als ältestes Kind von Elisabeth Glarmin-Flach und Dietrich Flach in Darmstadt geboren. Mein Vater ist Direktor des Sozialgerichtes in Darmstadt und meine Mutter arbeitet als Verwaltungsjuristin beim Regierungspräsidium in Darmstadt. Mein jüngerer Bruder Patrick Flach studiert Medizin.

Von 1996 bis 2000 besuchte ich die Friedrich-Ebert-Grundschule in Darmstadt. Anschließend wechselte ich auf das Gymnasium Edith-Stein-Schule in Darmstadt, wo ich 2009 die Allgemeine Hochschulreife mit einem Gesamtnotendurchschnitt von 1,8 erlangte.

Nach dem Abitur absolvierte ich ein Freiwilliges Soziales Jahr im DRK-Schul- und Therapiezentrum Raisdorf bei Kiel.

Im Wintersemester 2010 begann ich an der Georg-August-Universität Göttingen das Studium der Zahnmedizin, welches ich 2015 mit der Erlangung des Staatsexamens und der Gesamtnote „gut“ beendete.

Von September 2016 bis Juni 2017 arbeitete ich in der Zahnarztpraxis Dr. Claar und Kollegen in Kassel als Vorbereitungsassistentin. Seit Juli 2017 arbeite ich bei Dr. Mazurek in Fuldabrück bei Kassel als Vorbereitungsassistentin. 